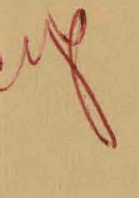

\title{
Contract Report
}

SC-CR-70-6092

December 1970

\section{RECEIVED BY DTIE DEC 291970}

\begin{abstract}
AN ANALYSIS OF DSBIFM
TELEMETRY SYSTEM ERRORS
\end{abstract}

Prepared by

Kenneth M. Uglow

Sarasota, Florida 


\section{DISCLAIMER}

This report was prepared as an account of work sponsored by an agency of the United States Government. Neither the United States Government nor any agency Thereof, nor any of their employees, makes any warranty, express or implied, or assumes any legal liability or responsibility for the accuracy, completeness, or usefulness of any information, apparatus, product, or process disclosed, or represents that its use would not infringe privately owned rights. Reference herein to any specific commercial product, process, or service by trade name, trademark, manufacturer, or otherwise does not necessarily constitute or imply its endorsement, recommendation, or favoring by the United States Government or any agency thereof. The views and opinions of authors expressed herein do not necessarily state or reflect those of the United States Government or any agency thereof. 


\section{DISCLAIMER}

Portions of this document may be illegible in electronic image products. Images are produced from the best available original document. 


\section{NOTICE}

This report was prepared as an account of work sponsored by the United States Government. Neither the United States nor the United States Atomic Energy Commission, nor any of their employees, nor any of their contractors, subcontractors, or their employees, makes any warranty, express or implied, or assumes any legal liability or responsibility for the accuracy, completeness or usefulness of any information, apparatus, product or process disclosed, or represents that its use would not infringe privately-owned rights.

Printed in the United States of America Available from

Clearinghouse for Federal Scientific and Technical Information National Bureau of Standards, U.S. Department of Commerce Springfield, Virginia 22151

Price: Printed Copy $\$ 3.00$; Microfiche $\$ 0.65$ 


\author{
Prepared by \\ Kenneth M. Uglow \\ Sarasota, F1orida \\ for \\ Sandia Laboratories \\ Albuquerque, New Mexico
}

Contract No. 72-8647

Consultant - C. S . Johnson, 7221

Buyer - R. D. Freyermuth, 4371

LEGAL NOTICE

This report was prepared as an account of work sponsored by the United States Government. Neither the United States nor the United States Atomic Energy Commission, nor any of their employees, nor any of their contractors, subcontractors, or their employees, makes any warranty, express or implied, or assumes any legal liability or responsibility for the accuracy, completeness or usefulness of any information, apparatus, product or process disclosed, or represents that its use would not infringe privately owned rights.

December 1970 


\section{TABLE OF CONTENTS}

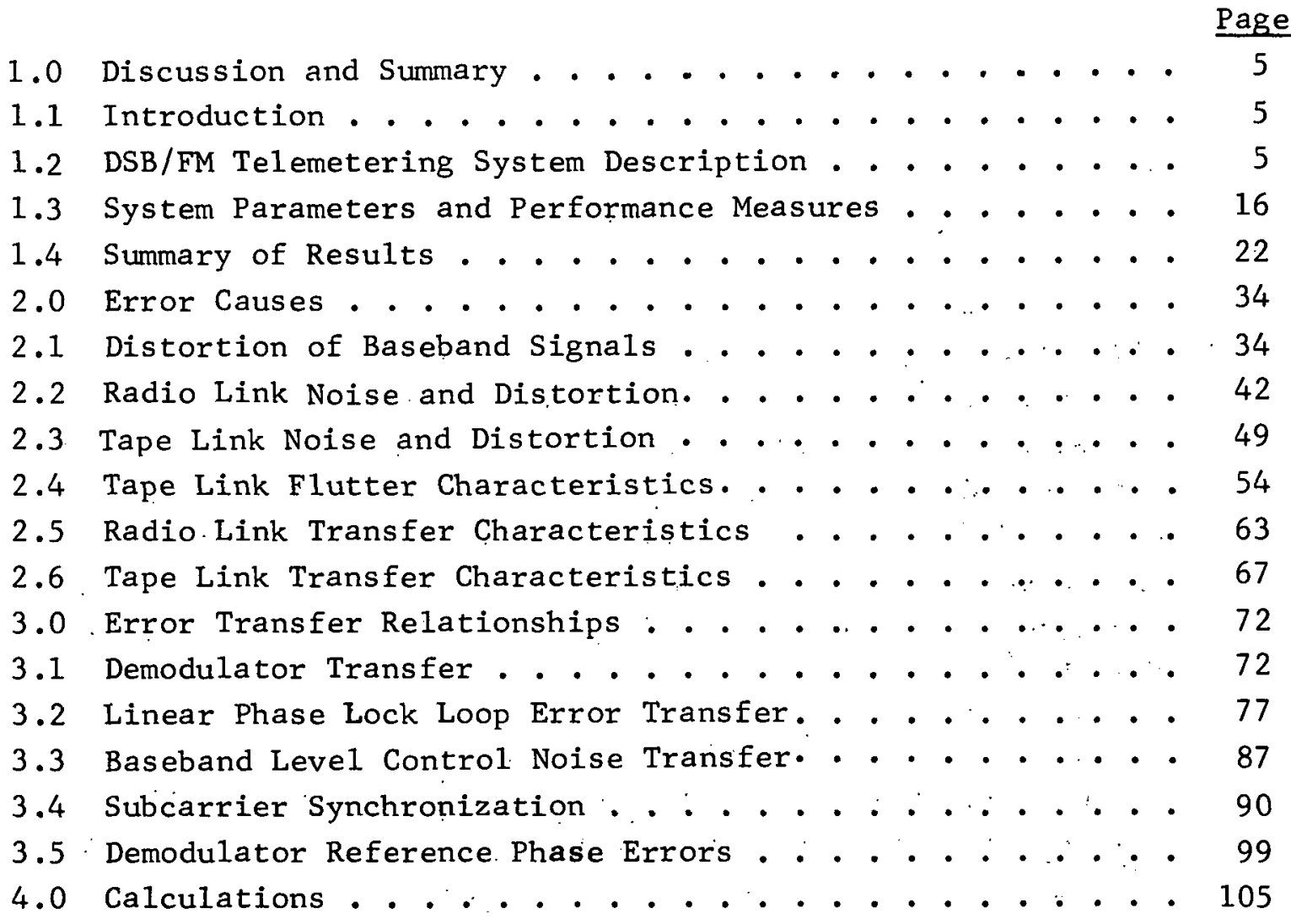




\section{ABSTRACT}

This report cuvers the result of a study of errors in two methods of radio telemetry using suppressed-carrier double-sideband subcarrier channels on an FM radio carrier, including the errors caused by magnetic tape recording and reproduction of the received signal. The two methods are the Independent Subcarrier Method (ISM) and the Harmonic Subcarrier Method (HSM) currently under consideration for standardization by the Telemetry Working Group of the Inter Range Instrumentation Group. The report deals with errors caused by the transmission and tape recording links.

Much of the report is devoted to the splection of analytical and empirical models for the disturbances caused by the transmission and recording links, including the presentation of exporimental data from several laboratories. Error transfer relationships are included for determining the error on the data channel output due to the disturbances. These relationships and the error cause models are then combined in tables of calculated values for many different combinations of recordiug methods, receiver carrier/noise ratios, channel locations, and suhrarrier methode. 


\subsection{DISCUSSION AND SUMMARY}

1.1 Introduction.

This report covers the results of a study of errors in two methods of radio telemetry using suppressed-carrier double-sideband subcarrier channels on an FM radio carrier, including the errors caused by magnetic tape recording and reproduction of the received signal. The two methods are the Independent Subcarrier Method (ISM) and the Harmonic Subcarrier Method (HSM) currently under consideration for standardization by the Telemetry Working Group of the Inter Range Instrumentation Group (IRIG). The report deals with errors caused by the transmission and tape recording links.

Much of the report is devoted to the selection of analytical and empirical models for the disturbances caused by the transmission and recording links, including the presentation of experimental data from several laboratories. Error transfer relationships are included'for determining the error on the data channel output due to the disturbances. These relationships and the error cause models are then combined in tables of calculated values for many different combinations of recording methods; receiver carrier/noise ratios, channel locations, and subcarrier methods. The results of these calculations are summarized and discussed in Section 1.4. The reader familiar with the methods and terminology may start with Section 1.3 which gives the system parameters used, afticr which Section 1.4 will suffice to show the findings.

The error cause models are developed in Sections 2.1 through 2.6, and the error transfer relationships in Sections 3.1 through 3.5. The calculations are outlined in Section 4.1.

\section{1.? DSR/FM Telemetering System Description}

In DSB/FM telemetering a multiplex of DSB subcarrier signals (and pilot signals) is transmitted by an FM transmitter. The combination of DSB signals and pilot signals is called the baseband signal in this report. The signal from the transmitter is received and demodulated in an FM receiver, which produces a somewhat faithful reproduction of the baseband signal which entered the transmitter. The demultiplex equipment 
following the receiver contains a subcarrier demodulator for each subcarrier channel present in the transmitting multiplex equipment, in addition to level (gain) control circuits which serve to standardize the end-to-end gain of a subcarrier channel.

A subcarrier signal is produced by a subcarrier modulator whose two inputs are a data signal and a reference signal. The data signal is the signal to be reproduced at the output of the distant receiving station. The reference signal is a constant-frequency sinusoid. Each channel in the multiplex has a different assigned referenre frequency. The output of the modulator is a signal whose frequency equals the reference frequency and whose amplitude is linearly proportional to the input data signal. The amplitude may go negative as well as positive, in which case the negative subcarrier amplitude may be envisioned as a 180 degree phase reversal. With zero data voltage into the modulator, the subcarrier output from the modulator is essentially zero. A modulator with these characteristics is called a suppressed-carrier doublesideband (DSB) modulator.

Reproduction of the input data signal by the subcarrier demodulator requires detection of the amplitude of the desired subcarrier signal. An ordinary AM detector (rectifier, for example) will not do the job because it cannot distinguish between the two possible phases of the subcarrier signal caused hy positive and negative data signals. A synchronous detector, with a demodulator reference signal input, if: required. The demodulator reference has the same frequency as the subcarrier modulator reference, and in phase-controlled so as to be essentially in phase with the desired one of the two possible phases of the subcarrier signal. The phase control function is called synchronization in this report. The demodulator in essence multiplies the subcarrier and reference signals and lowpass filters the product so as to pass the frequency components of the desired data signal. The filtered product is proportional to the amplitude of the subcarrier signal, which in turn (except for errors) is proportional to the data input to the subcarrier modulator.

Two approachs to subcarrier reference generation and synchronization are considered in this study. The harmonic subcarrier method (HSM) makes use of modulator reference frequencies which are exact harmonics of a base frequency, and are generated in a synthesizer from a single stable oscillator. The phase relationships among the several reference 
signal and pilot signals are predetermined and are used in the operation of the receiving station demultiplex equipment. Pilot signals transmitted in the baseband signal are used in synchronizing a frequency synthesizer in the demultiplexing equipment which provides the necessary reference signals for all of the demodulators.

The independent subcarrier method (ISM) does not require exact harmonic frequency relationships among the various modulator reference signals. Each subcarrier demodulator achieves its reference signal synchronization from its subcarrier signal input, without any connection with the other subcarrier channels. The problem of knowing which phase of the subcarrier signal represents positive data voltage is solved by transmitting a reference tone along with the data signal on each channel and comparing the polarity of the tone output from the demodulator with that of a similar tone transmitted unambiguously in the baseband. The hardware experience published to date has been mainly with independent subcarriers (References $1,2,12$ ).

Unlike FM subcarriers, (whose amplitudes at the multiplex output to the transmitter are steady) DSB subcarrier amplitudes vary in response to the data inputs. As a result the peak transmitter deviation due to a subcarrier is variable, and this quantity is not convenient for analyzing channel performance. It is possible to work with the mean square data inputs to all channels. The measure of frequency deviation is the Hertz. The rms deviation may be measured in Hertz, and the mean square deviation is therefore in Hertz. ${ }^{2}$. The mean-square is used frequently in this report because of the additive property of various sources of equivalent deviation caused by signals and noise. Mean square voltages at transmitter input, receiver output, recorder output, etc., could be used instead, but this would require the introduction of a number of conversion constants which would cancel out in the final error ratio calculations. Therefore wherever possible signal and noise levels are expressed in terms of equivalent transmitter modu1ation.

'l'he variation of input data activity to the various channels permits the use of automatic transmitter modulation control (TMC) to increase the modulation gain when the sum of the data input powers is low in order to achieve improved signal-to-noise ratios at the channel outputs, and to reduce the gain in case the total channel activity rises to unexpected levels (Reference 15). The use of TMC complicates the 
calculation of the performance of a particular channel somewhat because of the difficulty of predicting the nature of real-1ife data signals. In this report the problem is avoided by assuming all of the channels except the channel being analyzed (test channel) are loaded from independent random sources having equal mean square levels. It is assumed that the TMC holds the total mean square transmitter deviation at a fixed value. The deviation due to the test channel can then be calculated by finding the ratio of its mean square data input level to the sum of the mean square data input levels of all of the channels plus the pilot levels. Multiplying the mean square cotal cransmitier devidtion by this ratio gives the mean square deviation due to the test channel.

In order to restore the demodulator inputs to values representing the various data input values, the demultiplexing equipment must contain baseband level control functions. In addition to compensating for variations in radio link gain, the baseband level control (BLC) must compensate for the intentional gain variation due to the TMC. In order to accomplish this it is necessary to mix a BLC pilot signal of constant amplitude with the outputs of the subcarrier modulators. The BLC senses the amplitude of this pilot signal and controls the baseband gain to maintain it essentially constant.

The TMC senses and controls the level of the baseband signal entering the transmitter modulator, with a time constant of a fraction of a second. The desired time constant depends on the characteristics of the data signals to be handled. Sudden increases in data activity will overload the radio link if the time constant is too long. A short time constant, on the other hand, requires more rapid response on the part of the baseband level control in the demultiplexing equipment, which in turn makes the BLC more responsive to various noise error sources. The errors caused by the TMC and BLC due to data level variations are discussed in Reference 15, and are not considered further in this report. The noise errors due to the baseband level control are covered in this report for a value of BLC response characteristic of the equipment at Sandia. Calculations for other values of BLC response are readily carried out using the relationships presented.

The baseband pilot signal used for ambiguity resolution in the independent subcarrier method is also used for baseband level control. These functions are referred to separately in the work that follows 
and the pilot characteristics are listed separately, although in reality only one pilot is needed when all of the channel data output filters have the same bandwidth. When several different data bandwidths are used, a baseband pilot may be used for each bandwidth, in which case one of them may be used for the BLC function.

In the harmonic subcarrier method two baseband pilots are generally needed for synchronizing the reference frequency synthesizer in the demultiplex equipment. If they are both passed through the transmitter modulation control, either may be used as the BLC pilot. If one of them bypasses the TMC, the other may be used for BLC provided it passes through the TMC. In principle a single low-frequency pilot in the baseband could provide unambiguous phase synchronization by use of i.ts harmonics for demodulation reference signals. This approach puts unduly severe requirements on the signal-to-noise ratio of this pilot signal, as well as its phase accuracy. It also restricts the use of the low portion of the baseband for other types of signals such as PCM. Therefore it is recommended that the precision phase synchronization pilot be placed well up in the baseband. This results in many possible phase synchronization states and an additional pilot is necessary to identify the required state. The additional pilot is called the phase reset pilot in this report.

The channel pilot signals used in the independent subcarrier method for ambiguity resolution may also be used for controlling the end-to-end gain of each data channel, compensating for slow variations in modulators, baseband equipment, radio equipment, and demodulators. This function is called channel level control (CLC). In order to conserve data bandwidth this function is made slow in response, and therefore it does not eliminate the need for the faster baseband level control. The channel pilots are needed for ambiguity resolution in the harmonic subcarrier method, and their use for CLC is optional. If not used, the components throughout the system must be sufficiently stable to provide the desired end-to-end gain accurancy or otherwise inflight c.atihration will be needed.

It is usually necessary to record telemetry signals for future processing and analysis. This is commonly accomplished by recording the receiver IF signal (predetection recording) or the baseband signal output of the receiver (post detection recording). The study covers 
the effects of tape recording on the demodulator output data obtained when the recordings are played back into demultiplexing equipment.

Figure 1.2-1 is a block diagram of the multiplex transmitting equipment for the harmonic subcarrier method. Double lines are used where a multiplicity of signals are generated. The frequency synthesizer generates all of the necessary demodulator reference and pilot signals. The reference signals may be squarewave signals provided that the modulators contain bandpass output filters to remove harmonic signals from the multiplier outputs. The pilot signals which are mixed in the baseband must in general be sinusoidal to avoid harmonic interference with other signals, and pilot filters are shown for removing harmonics from the synthesizer outputs. The function of the various blocks have been previously discussed. The modulator and TMC functions are shown in detailed diagrams in this figure.

The data filter shown in the modulator aids in preventing high frequency data signal components from creating interference in adjacent channels. The bandpass output filter also attenuates adjacent channe1 interference. Its design however involves compromises between out-ofband spectrum levels and in-band signal distortion when the input data bandwidth is wider than the channel design capability.

The transmitter modulation control is similar to the automatic gain control used in radio receivers. Its design is more difficult, however, because it is not dealing with a simple sinusoidal signal. The baseband signal is composed of signals from many sources of various characteristics so that the choice of level detector and filter characteristics becomes more complicated than in the case of the receiver AGC. Some of the considerations involved are discussed in Reference 15 .

Figure 1.2-2 is a block diagram of a receiving station for harmonic subcarrier DSB telemetry. A conventional FM telemetry receiver is used, followed by tape recording if needed. The predetection recorder input is the receiver IF signal, assumed to come from the output of the limiter in the receiver IF with suitable down-conversion in frequency. Automatic gain control is not needed at this point. When post detection recording is used the receiver video output should be used without gain control, because the transmitter modulation control tends to stabilize the baseband signal energy at the receiver video output. This should result in effective utilization of the recorder dynamic range. 

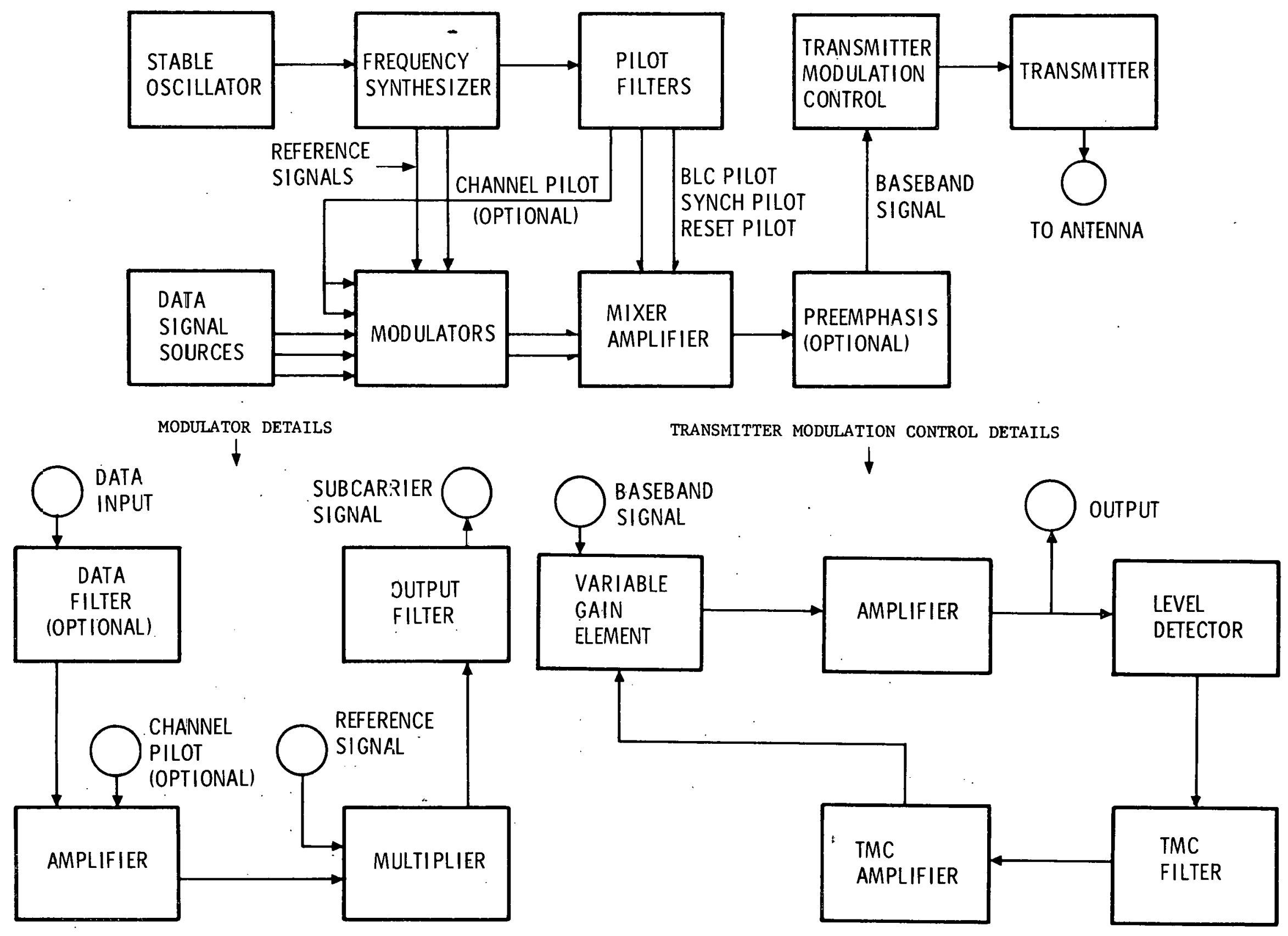


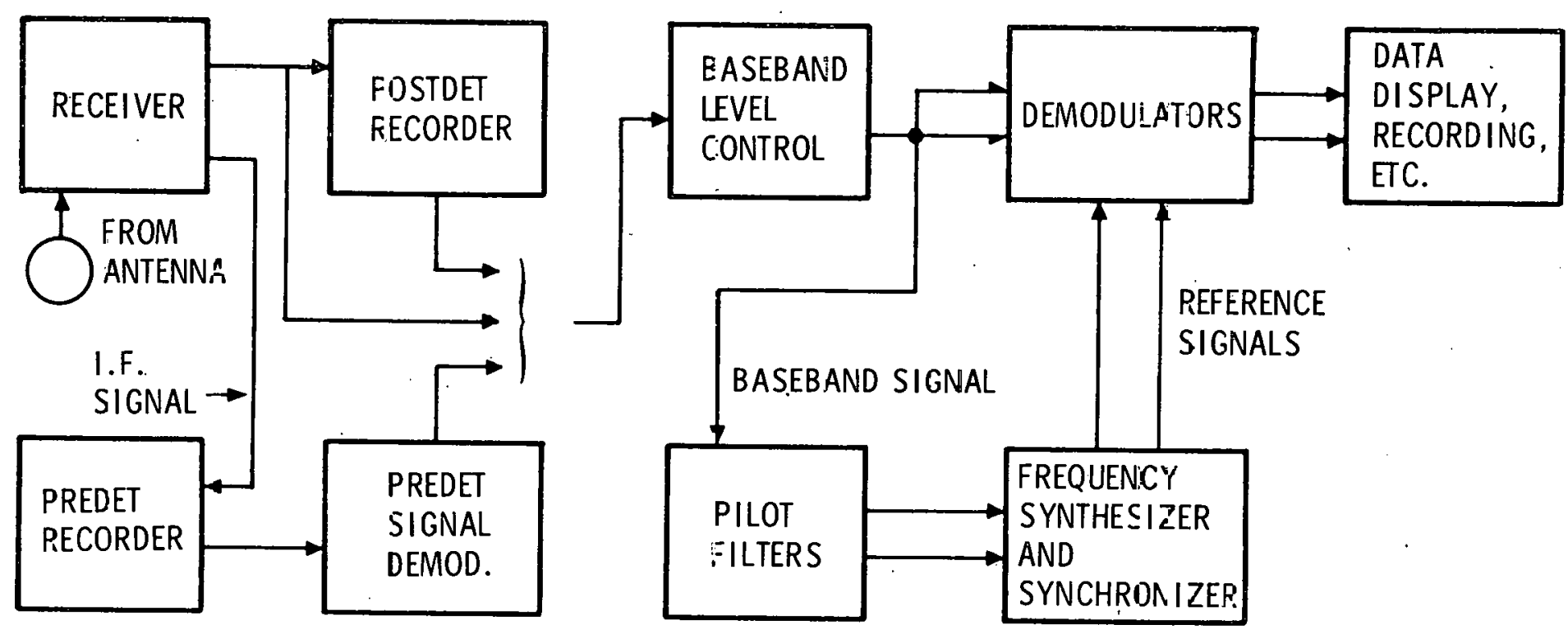

BASEBAND LEVEL CONTROL DETAILS

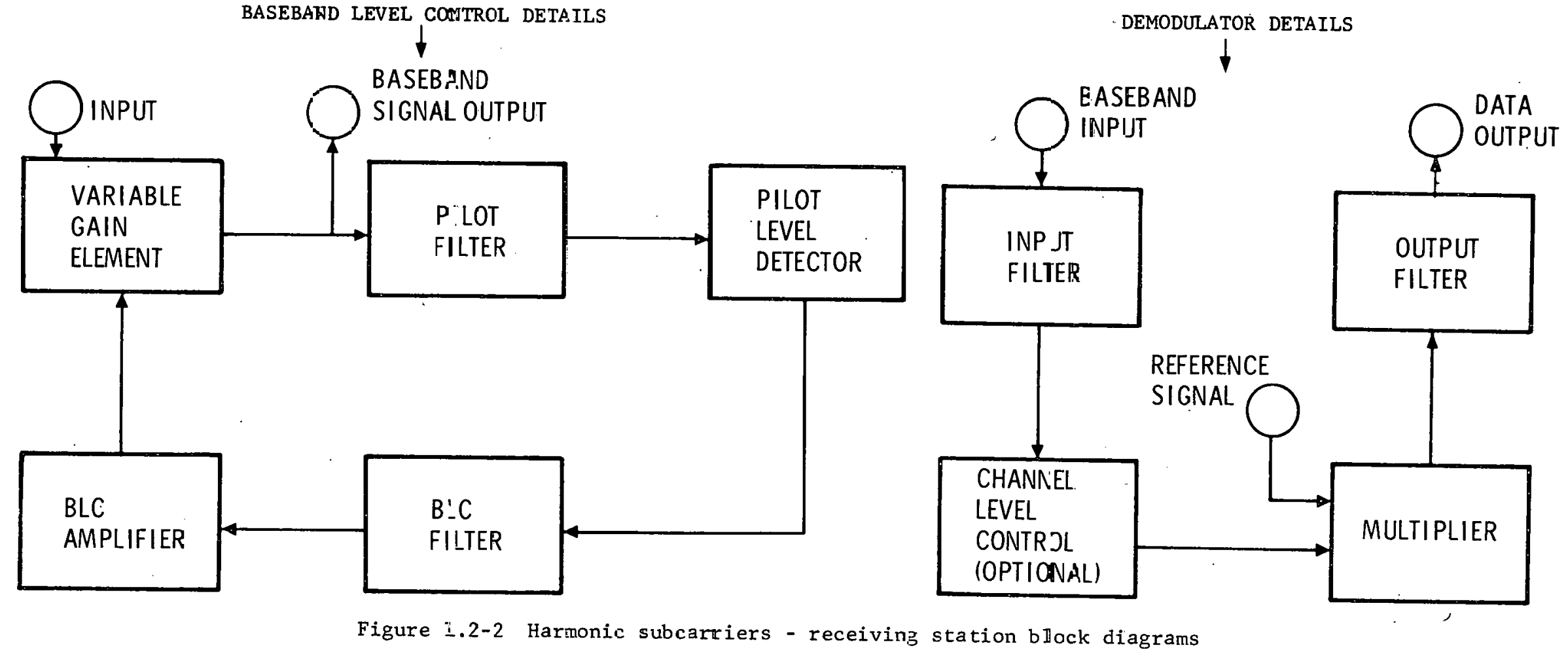

Figure i.2-2 Harmonic subcarriers - receiving station block diagrams 
The baseband level control is an automatic gain control which operates to keep the BLC pilot constant at its output, thereby restoring the various subcarrier signals to levels proportional to their respective data signal inputs. The total baseband signal at the BLC output then varies widely in the same. fashion as does the baseband signal input to the transmitter modulation control and is essentially an accurate replica of the latter. Pilot filters are shown for selecting the synchronization inputs to the frequency synthesizer, which in turn generates the demodulator reference signals.

Figure 1.2-3 is a block diagram of the multiplex transmitting equipment for independent subcarriers. In this case there is no frequency synthesizer. Instead each modulator module contains an independent reference signal oscillator. A pilot oscillator is shown which generates the baseband and channel signals needed for ambiguity resolution in the reference signal synchronization function of the receiving demultiplex equipment. The signal mixing and transmitter modulation control functions are similar to those used for harmonic subcarriers. The baseband pilot signal may be used both for baseband level control and for phase reset (ambiguity resolution). If the multiplex consists of channels with different data bandwidth capability more than one baseband pilot may be needed to accommodate more than one value of channel pilot frequency.

The receiving station block diagram is Figure 1..2-4. The receiver, recorder, and baseband level functions are the same as for harmonic subcarriers. There is no reference frequency synthesizer. Each demodulaLor uudule contalns 1 ts own reference oscillator and synchronization circuits. The demodulator diagram shows the channel level control function, although this is optional, as it is in the case of harmonic subcarriers.

The reference signal is generated by a phase lock 1oop (PLL) tracking the modulated subcarrier signal. The subcarrier signal is separated from the baseband with a bandpass filter. The filter output passes into a limiter. The limiter output defines the zero-crossings of the input wave. A frequency doubler operates on both the positiveand negative-going crossings. The doubler is used so that the phase lock loop can maintain synchronization when the polarity (phase) of the modulated sinewave subcarrier reverses. The oscillator in the PLL runs at twice the subcarrier frequency and therefore can accommodate either 

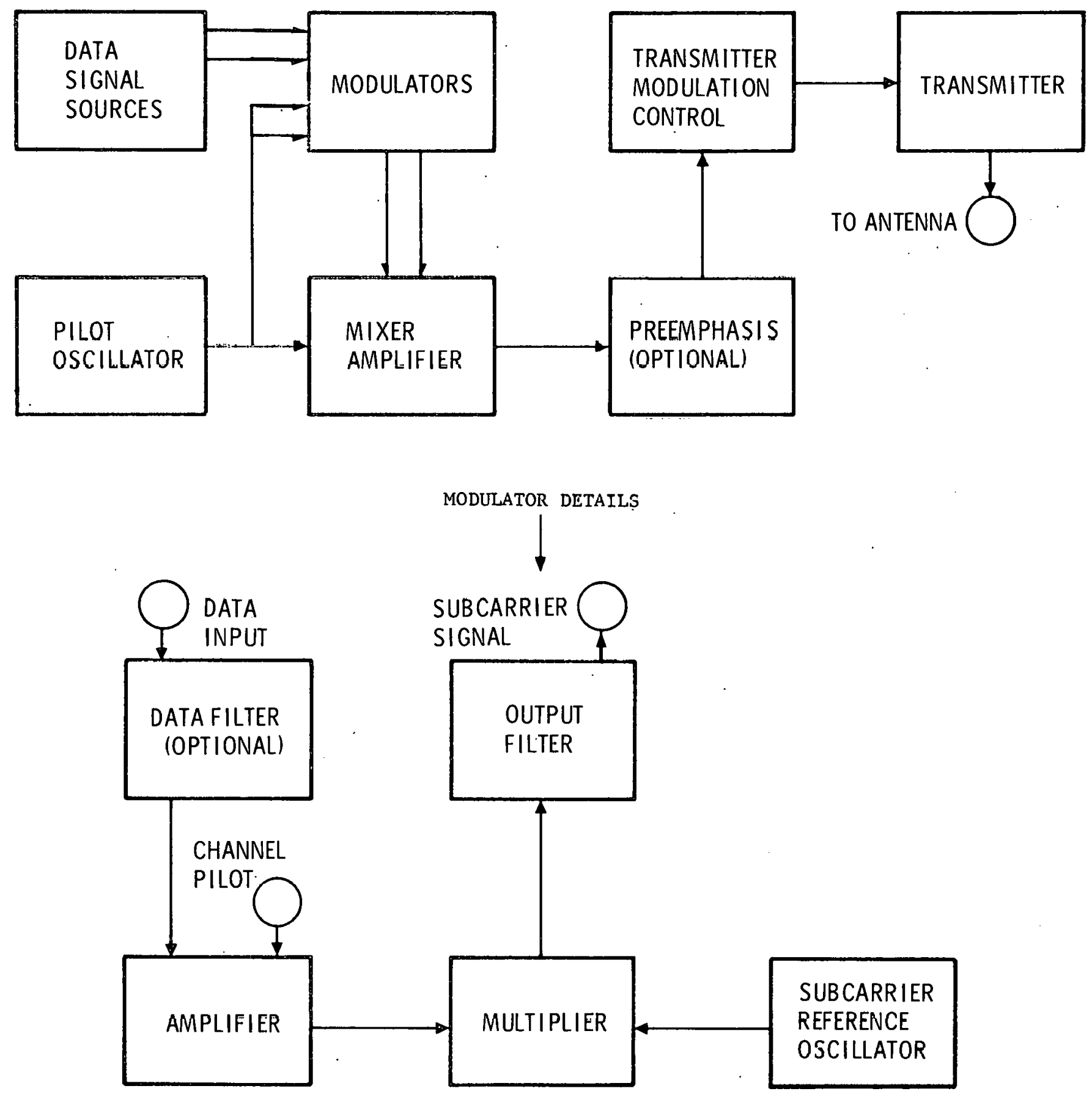

Figure 1.2-3 Independent subcarriers multiplex equipment block diagram 
BASEBAND
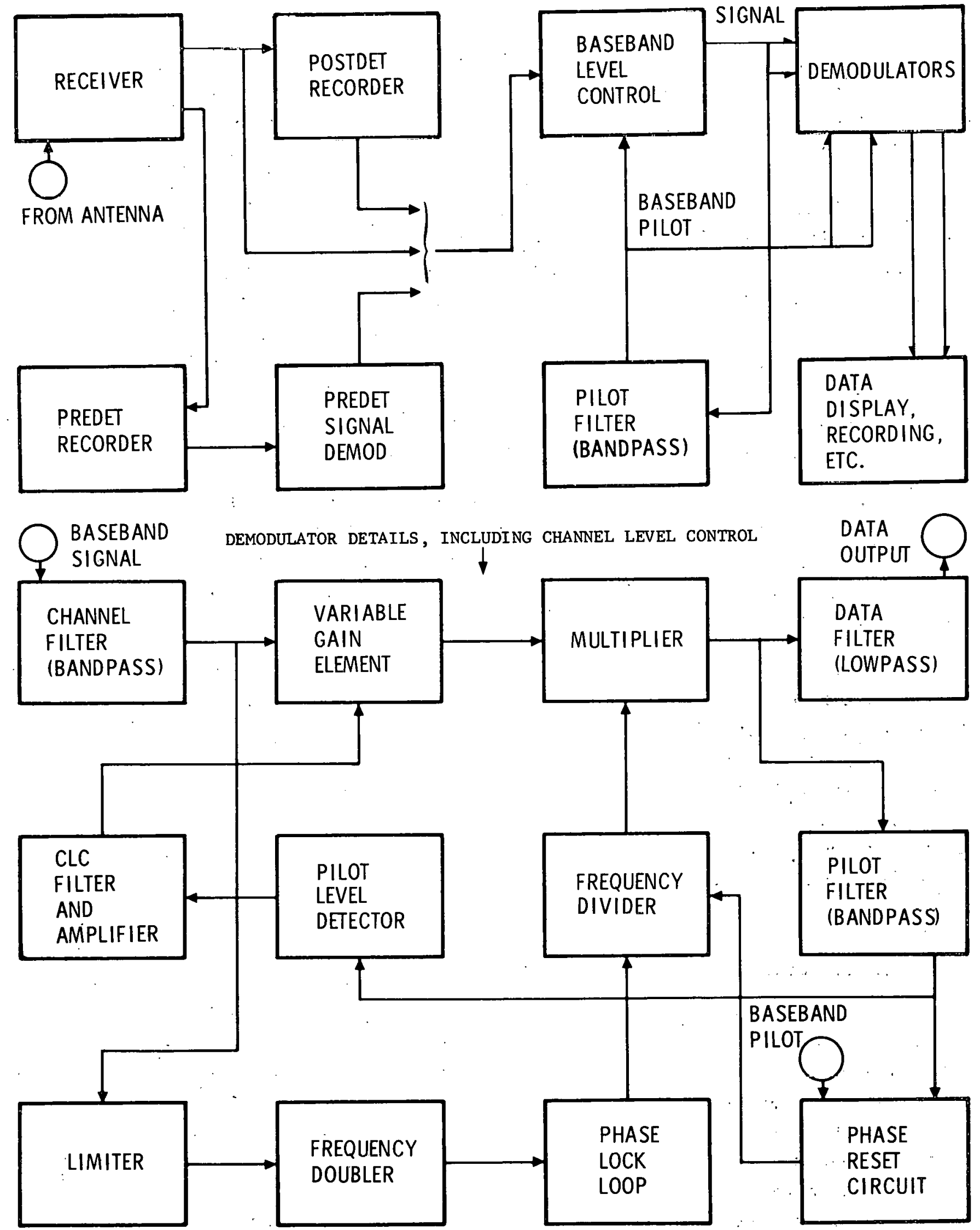

Figure 1.2-4 Independent subcarriers receiving station block diagram 
input phase without changing its own phase. In order to obtain the proper reference frequency, the PLL output signal operates a frequency divider whose output frequency equals the frequency of the modulated subcarrier signal input. The PLL and divider may be locked in either of two phases, one of which is incorrect and will result in inverted data output polarity. The incorrect phase is detected in the polarity reset circuit, whose inputs are pilot signals from the demodulated output and from the baseband. When these two pilot signals are of opposite phase, the frequency divider is given a reset signal which reverses its state. This reset will reverse the polarity of the reference signal input to the multiplier, which will in turn reverse the polarity of the pilot signal output from the demodulator to bring it in phase with the baseband pilot signal. When this occurs the reset circuit remains dormant until synchronization is lost for any reason.

The phase lock loop would lose lock if the subcarrier signal disappeared for an appreciable length of time compared to the PLL response time. This is avoided by having the channel pilot signal present, so that a subcarrier signal exists even when the data voltage input to the channel is zero.

The action of the channel levol control (GLC) is similar to that of the baseband level control, except that it is generally much slower because of the narrow band allocated to the CLC pilot. The pilot entering the modulator in the multiplex is controlled in amplitude accurately. The CLC circuit serves to control the demodulator input gain to keep this pilot at essentially constant amplitude at the output, and in doing so stabilizes the end-to-end gain of the channel for data signals as well as for the pilot.

\subsection{System Parameters and Performance Measures}

The parameters used in this study were taken Irum equipment procured by Sandia Laboratories for evaluation purposes. The present analysis predicts results to be expected in laboratory measurements on this equipment and will aid in explaining the physical effects responsible for results of future experiments. A parameter optimization analysis was not included in the study, but such an analysis can be carried. out making use of the relationships developed in this report. Some sources of error, such as modulator/demodulator nonlinearity, have 
not been covered. In many cases, these have been covered in the material referenced or in manufacturers specifications. The errors covered are caused by the overall system, including radio and tape recording/ playback links.

Each system configuration has twenty data channels, with $4 \mathrm{kHz}$ spacing between center frequencies. In the HSM system, the $64 \mathrm{kHz}$ channel slot is allocated to the phase synchronization pilot signal, and channel center frequencies run from $16 \mathrm{kHz}$ to $96 \mathrm{kHz}$. In the ISM system the $64 \mathrm{kHz}$ pilot is not needed. For convenience in tabulating error sources, the same top and bottom channel frequencies were used for the ISM system, although the top channel could have been moved down to $88 \mathrm{kHz}$. As a result the performance calculated for the top channel is slightly pessimistic.

The data response of all channels is taken as $1 \mathrm{kHz}$, being determined primarily by the lowpass output filter of the demodulators. The channel pilot frequency for the ISM system is $1.5 \mathrm{kHz}$, as is customary in current ISM equipment with $1 \mathrm{kHz}$ data output bandwidth. No channe1 pilots were used in the HSM analysis. Insofar as the analysis is concerned, their only effect was to use a small portion of the total baseband signal energy.

The nominal channel input voltage range was taken as plus and minus 2.5 volts, as a level to which other voltage levels could be referred. In a DSM/FM system the data inputs may exceed this range without harm, however. The subcarrier signal voltage corresponding to 2.5 volts dc input is called $\mathrm{E}_{2}$, and various pilot levels are referenced to the value $E_{2}$. These levels and other parameters are listed in Table $1.3-1$.

For the ISM system the baseband pilot (used for BLC and phase reset) is at $1.5 \mathrm{kHz}$ and has an amplitude $3 \mathrm{~dB}$ below $\mathrm{E}_{2}$. The channel pilot (used for CLC and phase reset) amplitude is set so that each of the two sidebands it produces are at a level $20 \mathrm{~dB}$ below $E_{2}$.

For the HSM system the phase reset pilot (also used for baseband level control) is placed at $4 \mathrm{kHz}$. Both the $4 \mathrm{kHz}$ pilot and the $64 \mathrm{kHz}$ pilot are set at levels $6 \mathrm{~dB}$ below the subcarrier level $\mathrm{E}_{2}$ so that their total energy is the same as that of the $1.5 \mathrm{kHz}$ pilot in the ISM case. 
TABLE $1.3-1$

\begin{tabular}{|c|c|c|c|}
\hline \multicolumn{2}{|l|}{ Type of Multiplex } & Independent & Harmonic \\
\hline Number of Channels & & 20 & 20 \\
\hline Top Channel Subcarrier & $\mathrm{kHz}$ & 96 & 96 \\
\hline Bottom Channel Subcarrier & $\mathrm{kHz}$ & 16 & 16 \\
\hline Outpue F1leẻr bändwidth & $\mathrm{Hz}$ & $1000^{\circ}$ & 1000 \\
\hline Receiver IF Bandwidth & $\mathrm{kHz}$ & 500 & 500 \\
\hline BLC Pilot Frequency & $\mathrm{kHz}$ & 1.5 & 4.0 \\
\hline CLC Pilot Frequency & $\mathrm{kHz}$ & 1.5 & -- \\
\hline Phase Synch Pilot Freq & $\mathrm{kHz}$ & --- & 64 \\
\hline Phase Reset Pilot Freq & $\mathrm{kHz}$ & 1.5 & 4.0 \\
\hline Demod. PLL Natura1 Freq & $\mathrm{Hz}$ & 150 & 150 \\
\hline Damping Factor & & 0.707 & 0.707 \\
\hline Noise Bandwidth & $\mathrm{Hz}$ & 471 & 471 \\
\hline BLC Loop 3 dB Bandwidth & $\mathrm{Hz}$ & 80 & 80 \\
\hline Noise Bandwidth & $\mathrm{Hz}$ & 126 & 126 \\
\hline Test Channel Input Type & & DC & $\mathrm{DC}$ \\
\hline Input Amplitude & $\mathrm{V}$ & 2.5 & 2.5 \\
\hline Subcarrier Leve 1 & $\mathrm{~V}$ & $\mathrm{~F}_{2}$ & $\mathrm{E}_{2}$ \\
\hline Other Channel Inputs, Type & & Random & Random \\
\hline Input Amplitude & RMS V & 1.6 & 1.6 \\
\hline BLC Pilot Level & $\mathrm{V}$ & $0.707 \quad E_{2}$ & $0.500 \mathrm{E}_{2}$ \\
\hline Phase Synch Pilot Lcvel & $\mathrm{v}$ & $-\ldots-$ & $0.500 \mathrm{E}_{2}$ \\
\hline Phase Reset Pilot Level & $\mathrm{V}$ & $0,707 \quad E_{2}$ & $0.5 n \cap \quad F_{2}$ \\
\hline CLC Pilot Sideband Level & $\mathrm{V}$ & $0.100 \mathrm{E}_{2}$ & --- \\
\hline Transmitter FM Preemphasis & & Flat & F1.nt \\
\hline RMS Transmitter Deviation & $\mathrm{kHz}$ & 80 & 80 \\
\hline RMS Test Channel Deviation & $\mathrm{kHz}$ & 25.6 & 26.4 \\
\hline BLC Pilot Deviation & $\mathrm{kHz}$ & 18.1 & 13.2 \\
\hline
\end{tabular}


The channel being analyzed for errors is referred to as the test channel. The tabulation of results contains both the $16 \mathrm{kHz}$ channel and the $96 \mathrm{kHz}$ channel as test channels. In each case, all of the other channels except the single test channel were loaded with random simulated data signals. The random input signal level was taken as 1.6 volts, which permits comparison with some experimental results from Reference 1: With this random input signal level it is obvious that frequent voltage excursions occur in excess of the channel nominal rating of 2.5 volts. The test channel input was taken as 2.5 volts dc. This test signal permits easy measurement of error values as compared to use of a random test sịgnal. It does not, however, give a measure of signal distortion due to the channel filters, nonlinearity, etc.

The carrier deviation due to the test channel has a value which depends upon the level of data signals into all channels as well as the pilot levels. We assume that the transmitter modulation control maintains the rms deviation due to the whole baseband signal constant. The rms deviation due to the test channel is a factor, $R$, times the total deviation, which was taken as $80 \mathrm{kHz} \mathrm{rms}$. The factor $\mathrm{R}$ is calculated as the ratio of the rms test channel level divided by the square root of the sum of the squares of the rms levels of all of the baseband signal components. The results of these calculations are plotted in the following figures. Figure 1.3-1 shows the ratio $R$ for the independent subcarrier multiplex, for both ten and twenty channels. For 1.6 volts rms signal into all channels but the test channel, and 2.5 volts dc into the test channel, the ratio is 0.32 , giving $25.6 \mathrm{kHz}$ rms carrier deviation for the test channel in the twenty-channel multiplex. For the harmonic subcarrier multiplex, the value of $\mathrm{R}$ for the parameters is given in Figure 1.3-2. For the 1.6 volt random input level to all other channels, the ratio $\mathrm{R}$ is 0.33 , giving $26.4 \mathrm{kHz}$ rms deviation for the test channel. Figure 1.3-3 is included to show the effect of increasing the pilot levels in the harmonic subcarrier multiplex to be equal to $\mathrm{E}_{2}$, the nominal subcarrier leve1. This reduces $\mathrm{R}$ to about 0.31 at the 1.6 volt level. It has more effect when the other channels have zero input, reducing $R$ from 0.82 in the previous case to 0.58 .

No transmitter modulation preemphasis was used in the analysis of this report, following the recommendations of several groups working with DSB and SSB subcarrier equipment. The reasons given are that the high frequency channel tends to dominate the transmitter modulation 
control function to the detriment of the lower channels, and that additional radio frequency spectrum width results.

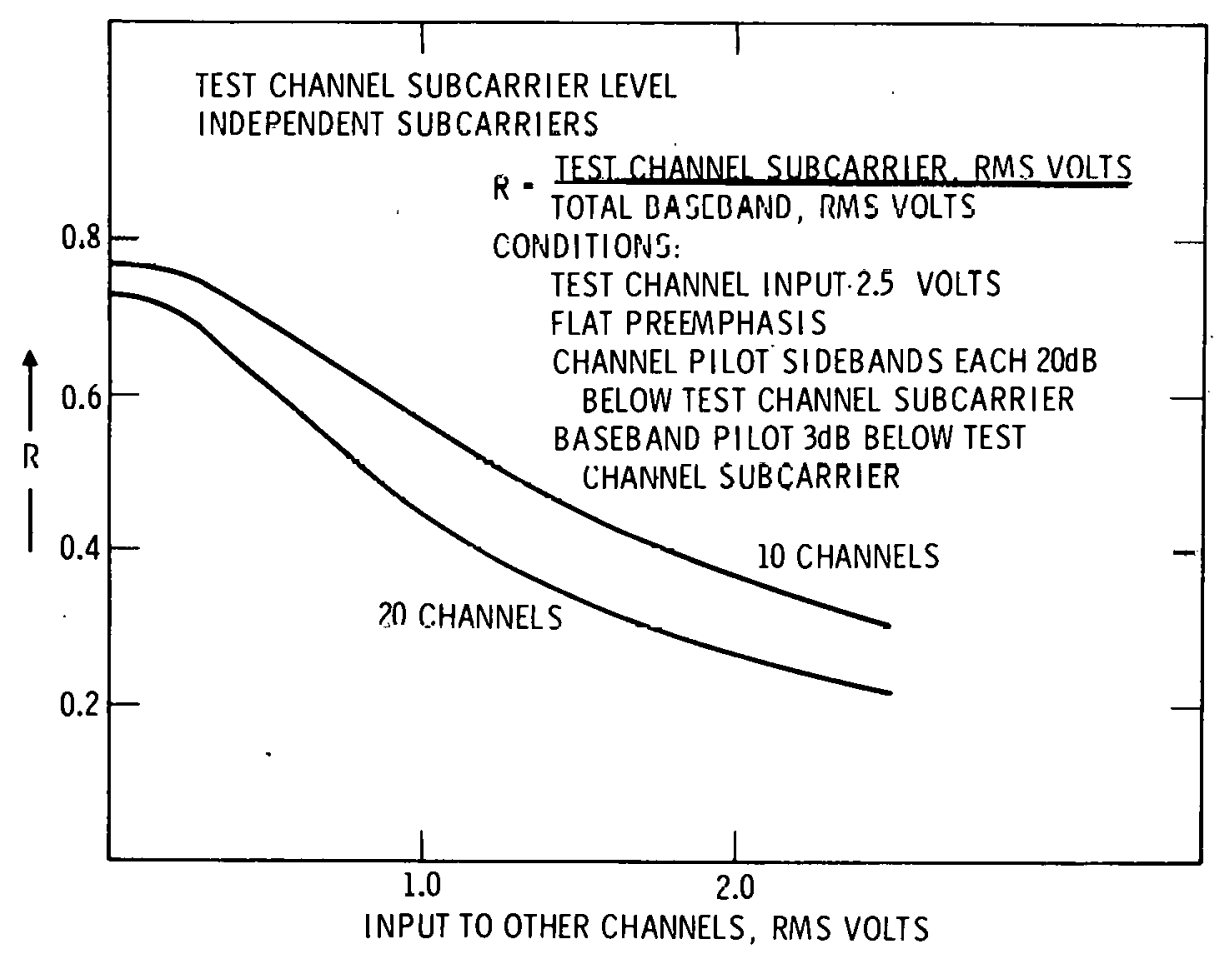

Figure 1.3-1 Test channel subcarrier level independent subcarriers 


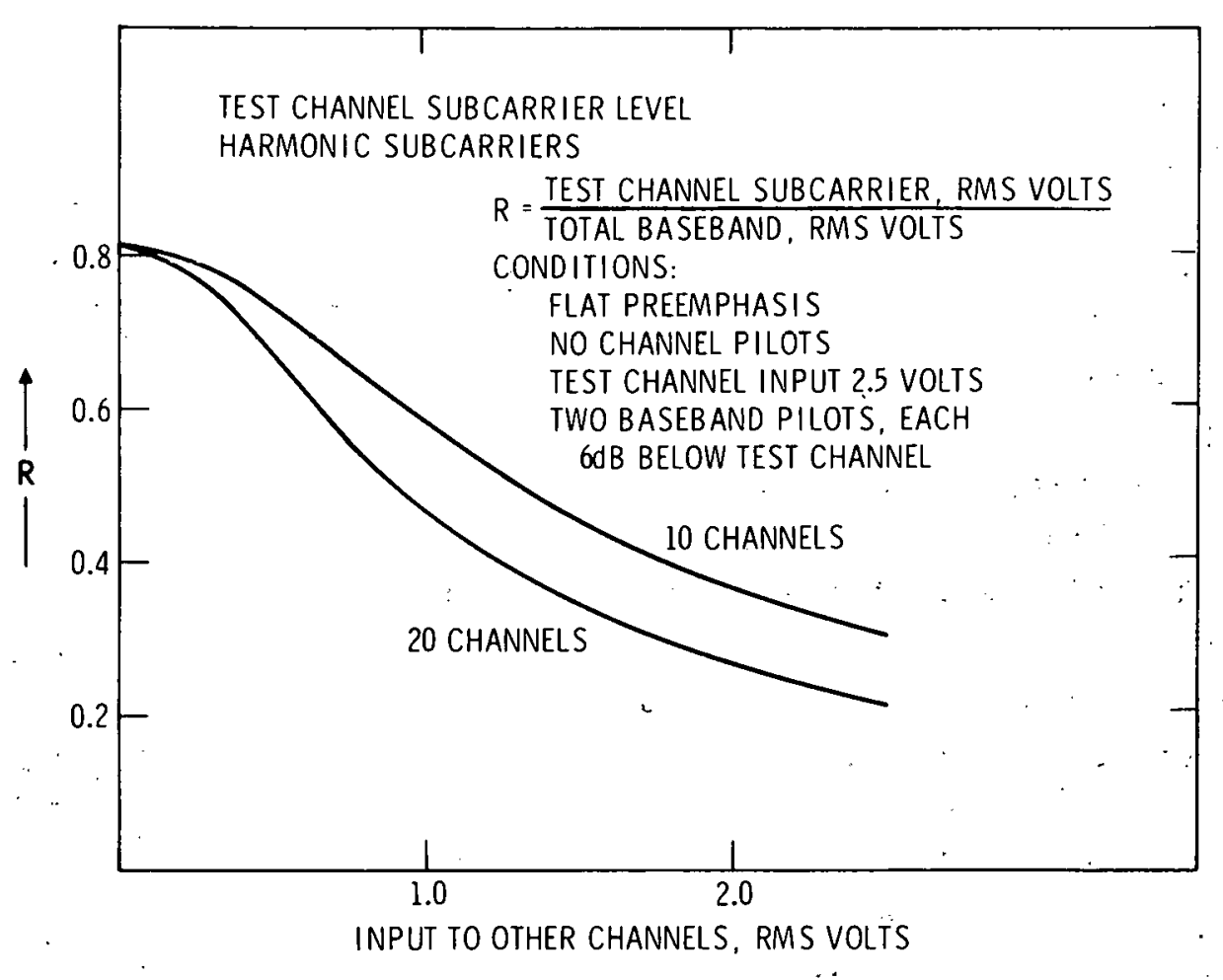

Figure 1.3-2 Test channel subcarrier level harmonic subcarriers

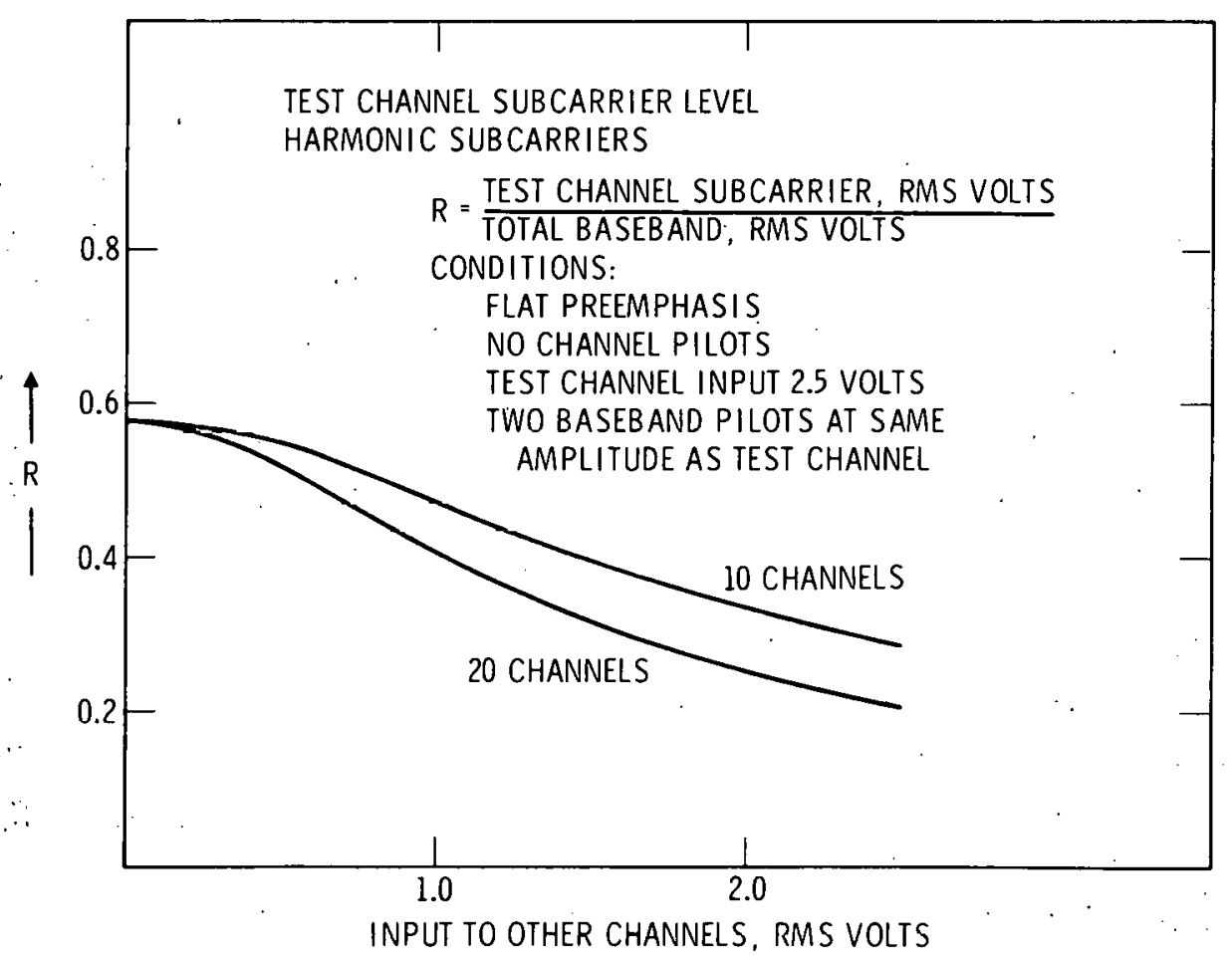

Figure 1.3-3 Test channel subcarrier level harmonic subcarriers 


\subsection{Summary of Results}

This section presents a summary of calculations of random errors for both harmonic and independent subcarriers, using the noise, distortion, and flutter models developed in Chapter 2. The noise and distortion models are summarized in Figure 1.4-1 for a set of random modulation parameters which simulate the DSB/FM baseband parameters. The tape link flutter models are summarized in Figure 2.4-5. A set of parameters which are assumed to represent the DSB equipment now at Sandia are listed in Table 1.3-1. That table may contain one or more inaccurate figures, because it was not possible to obtain measured quantities in all cases and some are matters of oplnion. This does not seriously detract from the value of the calculations, which bring out the significant error contributors under reasonable conditions without deriving an optimum set. One can then judge what changes might be made to achieve error reduction.

The errors are calculated for twenty-channel multiplexes with all but the test channel loaded with random data input at 1.6 volts rms. The test channel input is 2.5 volts. All channels have the same gain, without any transmitter modulation preemphasis.

The calculated errors are summarized in Tables 1.4-1 and 1.4-2 for the parameters of Table 1.3-1. These tables cover two recording modes, two types of subcarriers, two test-chanmel rrequencies, two IF signal-to-noise ratios, and four flutter models in addition to the flutter-free values, for a total of eighty error values. In these tables, the quantity $\mathrm{R}^{2}$ is the mean-square output error divided by the nominal full-scale rating of the channel. In case the nominal dc output is 2.5 volts for 2.5 volts input, the full scale value is defined as 5 volts. The quantity $100 \mathrm{R}$ is the rms error in percent of full scale.

The additive noise is the result of the noise and distortion models of Figure 1.4-1 translated by the demodulator and filtered by the lowpass data output filter. The BLC noise is the modulation of the output data voltage caused by baseband noise operating through the baseband level control servo. The PLL noise is due to the fluctuating component of phase error caused by the baseband noise and distortion models operating on the phase lock loop used for demodulator reference generation. The flutter error is the fluctuating error resulting from PLL phase error due to the error in tracking the time modulation 


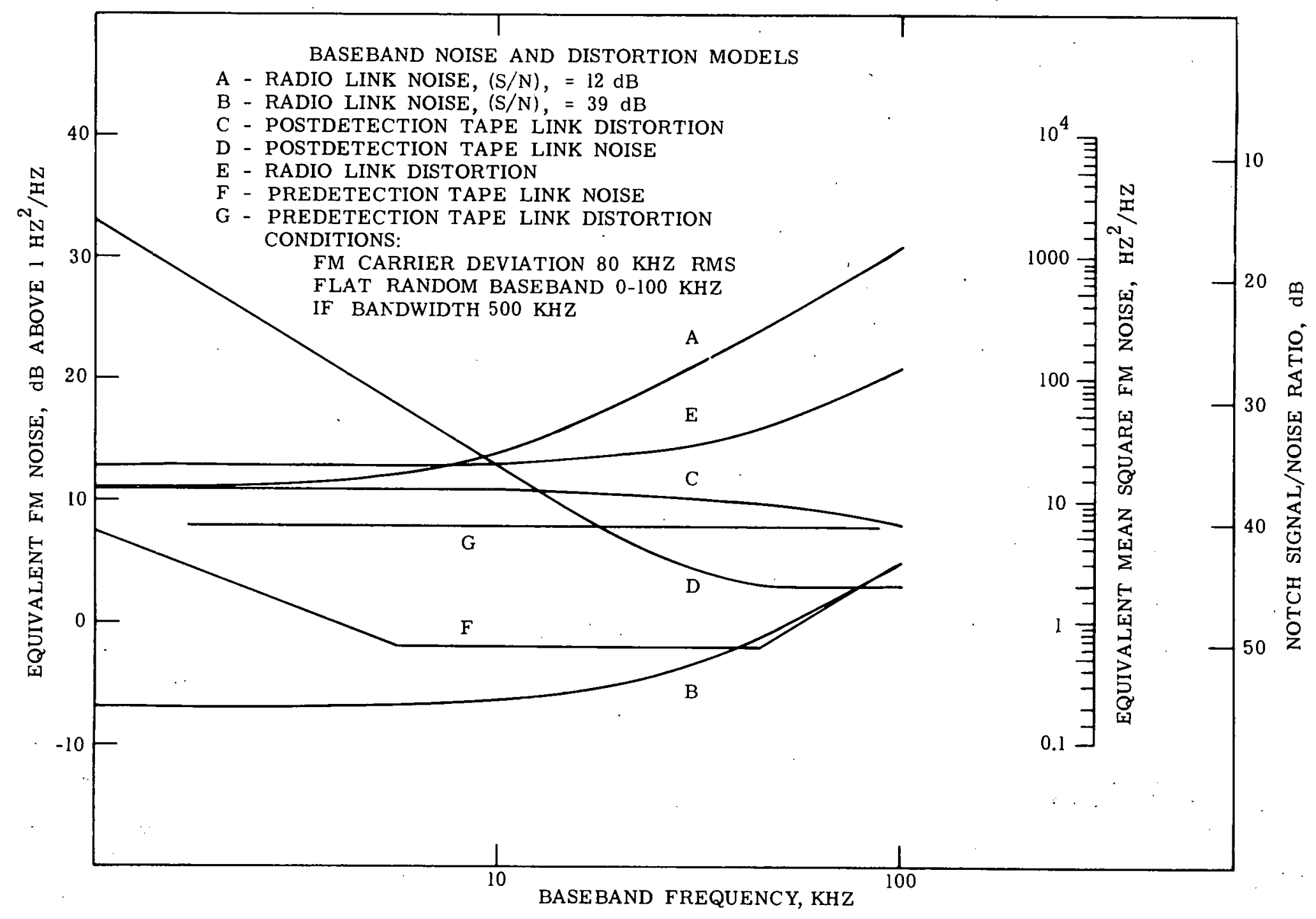




\begin{tabular}{|c|c|c|c|c|c|c|c|c|}
\hline \multicolumn{9}{|c|}{$\begin{array}{l}\text { RANDOM ERRORS FOR DSB/FM WITH PREDETECTION RECORDING } \\
\text { PARAMETER VAEVES ARE LISTED IN TABLE } 1.3-1\end{array}$} \\
\hline Recording Type & \multicolumn{8}{|c|}{ Predetect站on } \\
\hline Channel Frequency $\mathrm{kHz}$ & \multicolumn{2}{|c|}{16} & \multicolumn{2}{|c|}{96} & \multicolumn{2}{|c|}{16} & \multicolumn{2}{|c|}{96} \\
\hline IF $S / N$ & 12 & 39 & 12 & 39 & 12 & 39 & 12 & 39 \\
\hline 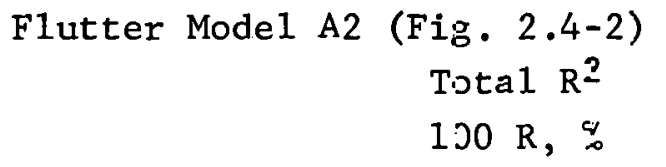 & $\begin{array}{r}0 \\
33 \\
0.58\end{array}$ & $\begin{array}{r}0 \\
15 \\
0.39\end{array}$ & $\begin{array}{r}14 \\
484 \\
2.20\end{array}$ & $\begin{array}{r}14 \\
65 \\
0.81\end{array}$ & $\begin{array}{r}0 \\
34 \\
J .58\end{array}$ & $\begin{array}{r}0 \\
16 \\
0.40\end{array}$ & $\begin{array}{r}54 \\
539 \\
2.32\end{array}$ & $\begin{array}{r}54 \\
110 \\
1.05\end{array}$ \\
\hline $\begin{aligned}\text { Flutter Mociel B (Fig. } 2.4-3) \\
\text { Total R } \\
100 \mathrm{R}, \%\end{aligned}$ & $\begin{array}{r}1 \\
34 \\
0.58\end{array}$ & $\begin{array}{r}1 \\
16 \\
0.40\end{array}$ & $\begin{array}{r}29 \\
499 \\
2.24\end{array}$ & $\begin{array}{r}29 \\
80 \\
0.90\end{array}$ & $\begin{array}{r}0 \\
34 \\
0.58\end{array}$ & $\begin{array}{r}0 \\
16 \\
0.40\end{array}$ & $\begin{array}{r}105 \\
590 \\
2.43\end{array}$ & $\begin{array}{r}105 \\
161 \\
1.27\end{array}$ \\
\hline $\begin{array}{r}\text { Flutter Mode1 C (Fig. } 2.4-\dot{4}) \\
\text { Tota1 R? } \\
100 \mathrm{R}, \%\end{array}$ & $\begin{array}{r}3 \\
36 \\
0.60\end{array}$ & $\begin{array}{r}3 \\
18 \\
0.43\end{array}$ & $\begin{array}{r}234 \\
704 \\
2.66\end{array}$ & $\begin{array}{r}234 \\
285 \\
1.69\end{array}$ & $\begin{array}{r}1 \\
35 \\
0.59\end{array}$ & $\begin{array}{r}1 \\
17 \\
0.41\end{array}$ & $\begin{array}{r}560 \\
1045 \\
3.24\end{array}$ & $\begin{array}{r}560 \\
616 \\
2.48\end{array}$ \\
\hline
\end{tabular}


TABLE $1.4-2$

\begin{tabular}{|c|c|c|c|c|c|c|c|c|}
\hline \multicolumn{9}{|c|}{$\begin{array}{l}\text { RANDOM ERRORS FOR DSB/FM WITH POSTDETECTION RECORDING } \\
\text { PARAMETER VALVES ARE LISTED IN TABLE } 1.3-1\end{array}$} \\
\hline Recording Type & \multicolumn{8}{|c|}{ Postdetection } \\
\hline Multiplex Type & \multicolumn{4}{|c|}{ Independent Subcarriers } & \multicolumn{4}{|c|}{ Harmonic Subcarriers } \\
\hline Channel Frequency $\mathrm{kHz}$ & \multicolumn{2}{|c|}{16} & \multicolumn{2}{|c|}{96} & \multicolumn{2}{|r|}{16} & \multicolumn{2}{|r|}{96} \\
\hline IF $\mathrm{S} / \mathrm{N}$ & 12 & 39 & 12 & 39 & 12 & 39 & 12 & 39 \\
\hline $\begin{array}{lr} & \text { TABLES : } \\
\text { Additive Noise } & (4.1-2) \\
\text { BLC Noise } & (4.1-3) \\
\text { PLL Noise } & (4.1-4) \\
& \text { Subtotal } \mathrm{R}^{2} \\
& 100 \mathrm{R}, \%\end{array}$ & $\begin{array}{r}10^{-6} \\
34 \\
92 \\
0 \\
126 \\
1.12\end{array}$ & $\begin{array}{r}10^{-6} \\
16 \\
91 \\
0 \\
107 \\
1.04\end{array}$ & $\begin{array}{r}\times 10^{-6} \\
465 \\
92 \\
2 \\
559 \\
2.36\end{array}$ & $\begin{array}{r}\times 10^{-6} \\
49 \\
91 \\
0 \\
140 \\
1.18\end{array}$ & $\begin{array}{r}10^{-6} \\
32 \\
31 \\
0 \\
63 \\
0.79\end{array}$ & $\begin{array}{r}\times 10^{-6} \\
15 \\
29 \\
0 \\
44 \\
0.66\end{array}$ & $\begin{array}{r}10^{-6} \\
436 \\
31 \\
91 \\
558 \\
2.36\end{array}$ & $\begin{array}{r}\times 10^{-6} \\
46 \\
29 \\
10 \\
85 \\
0.92\end{array}$ \\
\hline $\begin{array}{c}\text { Flutter Model A1 (Fig. } 2.4-2) \\
\text { Tota1 R2 } \\
100 \mathrm{R}, \%\end{array}$ & $\begin{array}{r}1 \\
127 \\
1.13\end{array}$ & $\begin{array}{r}1 \\
108 \\
1.04\end{array}$ & $\begin{array}{r}81 \\
640 \\
2.54\end{array}$ & $\begin{array}{r}81 \\
221 \\
1.49\end{array}$ & $\begin{array}{r}2 \\
65 \\
0.81 \\
\end{array}$ & $\begin{array}{r}2 \\
46 \\
0.68\end{array}$ & $\begin{array}{r}435 \\
993 \\
3.16\end{array}$ & $\begin{array}{r}435 \\
520 \\
2.28 \\
\end{array}$ \\
\hline $\begin{array}{r}\text { F1utter Model A2 (Fig. } 2.4-2) \\
\text { Tota1 } \mathrm{R}^{2} \\
100 \mathrm{R}, \%\end{array}$ & $\begin{array}{r}0 \\
126 \\
1.12\end{array}$ & $\begin{array}{r}0 \\
107 \\
1.04\end{array}$ & $\begin{array}{r}14 \\
573 \\
2.40\end{array}$ & $\begin{array}{r}14 \\
154 \\
1.24\end{array}$ & $\begin{array}{r}0 \\
63 \\
0.79\end{array}$ & $\begin{array}{r}0 \\
44 \\
0.66\end{array}$ & $\begin{array}{r}102 \\
660 \\
2.57\end{array}$ & $\begin{array}{r}102 \\
187 \\
1.37\end{array}$ \\
\hline $\begin{array}{r}\text { F1utter Mode1 B (Fig. } 2.4-3) \\
\text { Total } \mathrm{R}^{2} \\
100 \mathrm{R}, \%\end{array}$ & $\begin{array}{r}1 \\
127 \\
1.13\end{array}$ & $\begin{array}{r}1 \\
108 \\
1.04\end{array}$ & $\begin{array}{r}29 \\
588 \\
2.42\end{array}$ & $\begin{array}{r}29 \\
169 \\
1.30\end{array}$ & $\begin{array}{r}1 \\
64 \\
0.80\end{array}$ & $\begin{array}{r}1 \\
45 \\
0.67\end{array}$ & $\begin{array}{r}200 \\
758 \\
2.77\end{array}$ & $\begin{array}{r}200 \\
285 \\
1.69\end{array}$ \\
\hline $\begin{array}{r}\text { Flutter Model C (Fig. } 2.4-4) \\
\text { Total } \mathrm{R}^{2} \\
100 \mathrm{R}, \%\end{array}$ & $\begin{array}{r}3 \\
129 \\
1.14\end{array}$ & $\begin{array}{r}3 \\
110 \\
1.05\end{array}$ & $\begin{array}{r}234 \\
793 \\
3.24\end{array}$ & $\begin{array}{r}234 \\
374 \\
1.94\end{array}$ & $\begin{array}{r}3 \\
66 \\
0.81\end{array}$ & $\begin{array}{r}3 \\
47 \\
0.69\end{array}$ & $\begin{array}{r}940 \\
1498 \\
3.88\end{array}$ & $\begin{array}{r}940 \\
1025 \\
3.21\end{array}$ \\
\hline
\end{tabular}


associated with tape link flutter. Table 1.4-3 lists the overall errors for all of the previous conditions (except the flutter-free) with the primary contributors identified in each case. The terms flutter and TBE refer to the same error, although the two quantities have different dimensions, as explained in Section 2.4 and Equation 2.4-6.

Finally, the calculations for the parameters of Table 1.3-1 are presented graphically in Figures $1.4-2$ and 1.4-3, where the horizontal scales indicate different flutter models rather than quantitative dimensions. As Table 1.4-3 indicates, a discussion of all the points would be rather lengthy, but a few observations are easily made from the charts.

The $16 \mathrm{kHz}$ channel is relatively unaffected by flutter. With predetection recording, the radio link distortion accounts for 0.4 percent error, which rises to 0.6 percent with the low carrier-to-noise ratio in the IF (12 dB). There is little difference between harmonic and independent subcarrier performance. With postdetection recording, the error is increased appreciably as a result of modulation of the output by the baseband level control. In this case the modulation is due to the tape link noise operating through the BLC. The effect is larger in the independent subcarrier case because the pilot is at $1.5 \mathrm{kHz}$ as contrasted to $4 \mathrm{kHz}$ in the harmonic case. The tape link power spectrum has a strong $1 / \mathrm{f}^{2}$ content in this region.

The situation is more complicated in the $96 \mathrm{kHz}$ channel. To begin with, both the distortion and noise due to the radio link are stronger at $96 \mathrm{kHz}$ than at $16 \mathrm{kHz}$, so that for a carrier-to-noise ratio of $12 \mathrm{~dB}$ the radio link noise causes most of the error (in the absence of flutter) in both systems and with both recording methods.

When the carrier-to-noise ratio is large (39 $\mathrm{dB}$ ) radio link distortion accounts for about 0.7 percent error in the predetection recording mode. For harmonic subcarriers this rises to 0.9 percent in going to postdetection recording because of the BLC noise. For the independent subcarriers, this rises to 1.2 percent for postdetection recording because of the lower frequency of the BLC pilot in the tape 1ink noise sperctrum.

The $96 \mathrm{kHz}$ channel is definitely flutter sensitive, with the harmonic subcarrier system being more susceptible with the parameters of Table 1.3-1, insofar as the linear PLL assumption is concerned, which assumption is inherent in Figures 1.4-2 and 1.4-3. The curves 
TABLE $1.4-3$

\begin{tabular}{|c|c|c|c|c|}
\hline \multicolumn{5}{|c|}{ RANDOM ERROR SUMMARY FOR PARAMETERS OF TABLE 1.3-1 } \\
\hline $\begin{array}{l}\text { CHAN } \\
\mathrm{fz} \\
\mathrm{kHz}\end{array}$ & $\begin{array}{l}\operatorname{IF} \\
(\mathrm{S} / \mathrm{N}) \\
\mathrm{dB}\end{array}$ & $\begin{array}{l}\text { TBE } \\
\text { Mod }\end{array}$ & $\begin{array}{c}\text { Independent Subcarrier } \\
\text { RMS Error, } 100 \mathrm{R}, \% \\
\% \quad \text { Main Cause }\end{array}$ & $\begin{array}{l}\text { Harmonic Subcarrier } \\
\text { RMS Error, } 100 \mathrm{R}, \% \\
\% \quad \text { Main Cause }\end{array}$ \\
\hline & & & \multicolumn{2}{|c|}{ Predetection Recording } \\
\hline \multirow[t]{2}{*}{16} & 12 & $\begin{array}{l}\text { A1 } \\
\text { A2 } \\
\text { B } \\
\text { C } \\
\text { A1 } \\
\text { A2 } \\
\text { B } \\
\text { C } \\
\text { A1 } \\
\text { A2 } \\
\text { B } \\
\text { C } \\
\text { A1 } \\
\text { A2 } \\
\text { B } \\
\text { C }\end{array}$ & $\begin{array}{l}\text { 1.2 } \text { TBE, Radio link dist'n } \\
0.8 \text { Radio link dist'n } \\
0.9 \text { TBE, Radio link dist'n } \\
\text { 1.7 TBE } \\
2.4 \text { Radio link noise } \\
2.2 \text { Radio link noise } \\
2.2 \text { Radio link noise } \\
2.7 \text { TBE, Radio link noise } \\
0.4 \text { Radio link distortion } \\
0.4 \text { Radio link distortion } \\
0.4 \text { Radio link distortion } \\
0.4 \text { Radio link distortion } \\
0.6 \text { Radio link noise, dist'n } \\
0.6 \text { Radio link noise, dist'n } \\
0.6 \text { Radio link noise, dist'n } \\
0.6 \text { Radio link noise, dist'n }\end{array}$ & $\begin{array}{ll}\text { 1.7 } & \text { TBE } \\
\text { 1.1 } & \text { TBE, Radio link dist'n } \\
\text { 1.3 } & \text { TBE } \\
2.5 & \text { TBE } \\
2.7 & \text { Radio link noise } \\
2.3 & \text { Radio link noise } \\
2.4 & \text { Radio link noise } \\
3.2 & \text { TBE, Radio link noise } \\
0.4 & \text { Radio link distortion } \\
0.4 & \text { Radio link distortion } \\
0.4 & \text { Radio link distortion } \\
0.4 & \text { Radio link distortion } \\
0.6 & \text { Radio link noise, dist'n } \\
0.6 & \text { Radio link noise, dist'n } \\
0.6 & \text { Radio link noise, dist'n } \\
0.6 & \text { Radio link noise, dist'n }\end{array}$ \\
\hline & & & \multicolumn{2}{|c|}{ Postdetection Recording } \\
\hline 16 & 12 & $\begin{array}{l}\text { A1 } \\
\text { A2 } \\
\text { B } \\
\text { C } \\
\text { A1 } \\
\text { A2 } \\
\text { B } \\
\text { C } \\
\text { A1 } \\
\text { A2 } \\
\text { B } \\
\text { C } \\
\text { A1 } \\
\text { A2 } \\
\text { B } \\
\text { C }\end{array}$ & 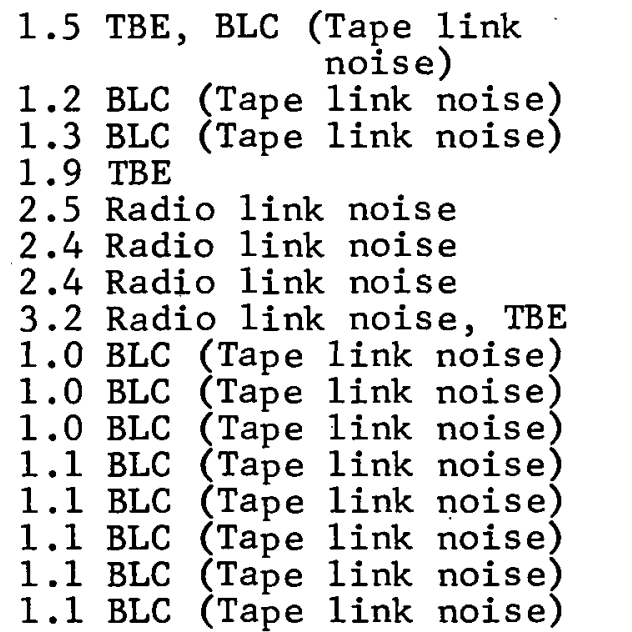 & $\begin{array}{ll}2.3 & \text { TBE } \\
1.4 & \text { TBE } \\
1.7 & \text { TBE } \\
3.2 & \text { TBE } \\
3.2 & \text { TBE, Radio link noise } \\
2.6 & \text { Radio link noise } \\
2.8 & \text { Radio link noise } \\
3.9 & \text { TBE, Radio link noise } \\
0.7 & \text { BLC, Radio 1ink dist'n } \\
0.7 & \text { BLC, Radio link dist'n } \\
0.7 & \text { BLC, Radio link dist'n } \\
0.7 & \text { BLC, Radio 1ink dist'n } \\
0.8 & \text { BLC, Radio link noise } \\
0.8 & \text { BLC, Radio link noise } \\
0.8 & \text { BLC, Radio link noise } \\
0.8 & \text { BLC, Radio link noise }\end{array}$ \\
\hline
\end{tabular}




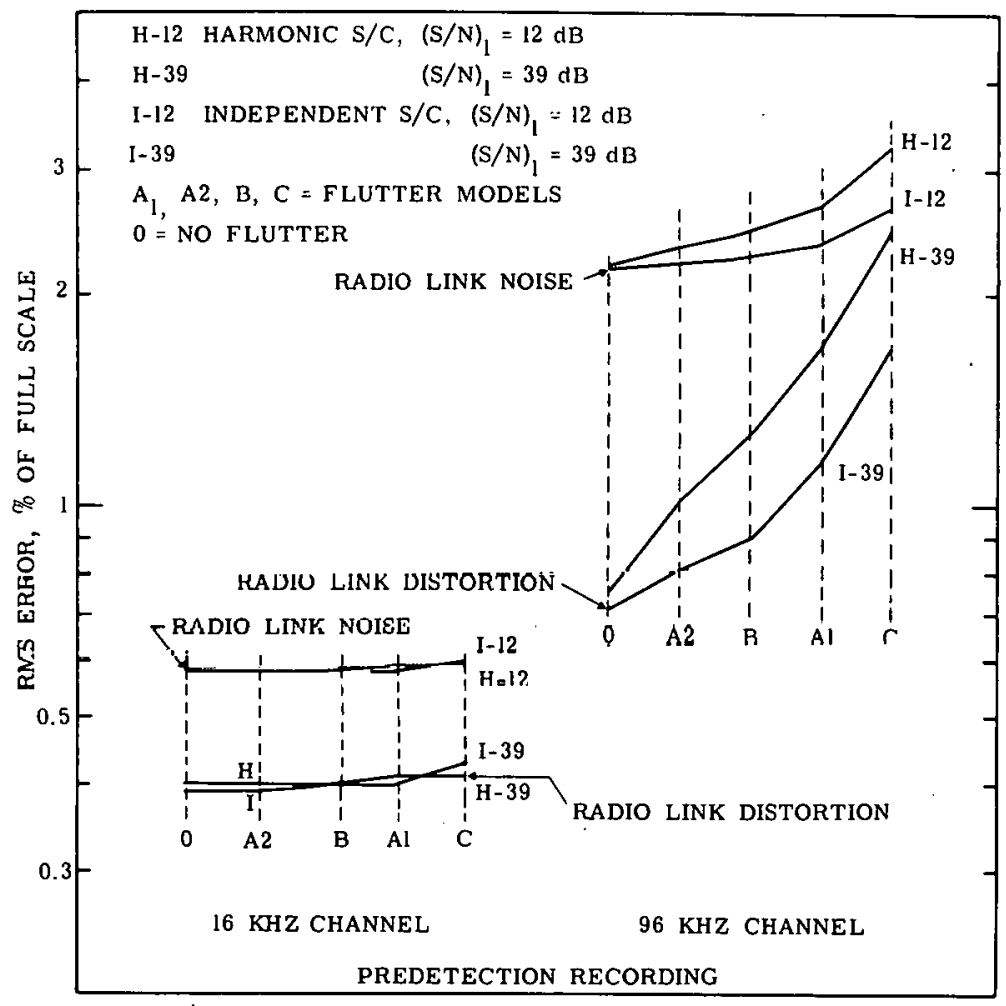

Figure 1.4-2

Random errors for predetection recording mode

Figure 1.4-3

Random errors for postdetection recording mode

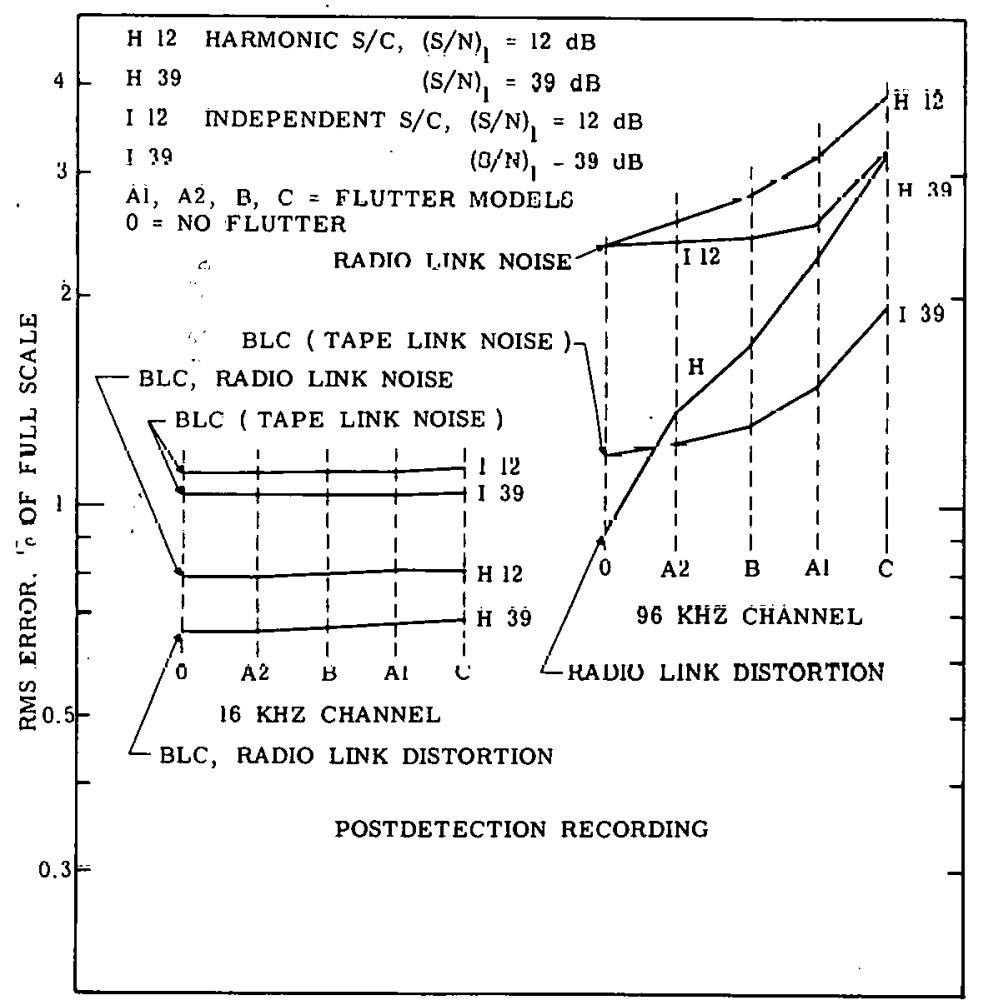


labelled I-39, for independent subcarriers with $39 \mathrm{~dB}$ carrier-to-noise ratio, are valid for all of the flutter models. The $\mathrm{C}$ model is thought to drive the PLL to the edge of unlock, as discussed in Section 3.4. As a result, the lower carrier-to-noise ratio (12 dB) will probably cause unlock at an annoying rate with the $\mathrm{C}$ model. In addition, for lower data voltage inputs, the loop signal-to-noise ratio decreases and unlocks will occur yet more frequently: As a result, the I-12 curve is somewhat artificial. The H-12 curve has more validity, for two reasons. First, the pilot signal level does not drop below its nominal value (unless the total data power load is larger than nominal). Second, the PLL is operating at $64 \mathrm{kHz}$ rather than twice $96 \mathrm{kHz}$, so it can tolerate three times the time base error amplitude without unlock than can the independent subcarrier PLL. As a result, unlock due to noise or flutter is expected to be infrequent on curve $\mathrm{H}-12$, for the harmonic subcarriers at $12 \mathrm{~dB}$ carrier-to-noise ratio.

The larger error on the harmonic subcarrier curves at $96 \mathrm{kHz}$ is due to the steady phase errors between the two demodulator inputs, as compared to the independent case. The phase error sources are discussed in Section 3.5, and their effects in Section 3.1. The harmonic subcarrier performance at $96 \mathrm{kHz}$ can be improved by increasing the PLL natural frequency. A partial set of calculations for other values are tabulated in Table 1.4-4 and plotted in Figure 1.4-4. It is assumed for these figures that the phase synchronization pilot power at $64 \mathrm{kHz}$ is increased in proportion to the loop bandwidth in order to keep the PLL phase noise unchanged, although this is not necessary. Then the pilot signal amplitude is $0.5 \mathrm{E}_{2}$ for $\mathrm{f}_{\mathrm{n}}$ at $150 \mathrm{~Hz}, 0.7 \mathrm{E}_{2}$ at $300 \mathrm{~Hz}$, and $0.9 \mathrm{E}_{2}$ at $500 \mathrm{~Hz}$, where $\mathrm{E}_{2}$ is the subcarrier amplitude corresponding to 2.5 volts data input to the test channel. Figure 1.4-4 shows the advantage of using the wider loop.

The question of improving the predicted unlock characteristics of the PLL for the independent subcarriers is not so easy to discuss. Quantitative data on the actual unlock conditions is completely lacking. It does not show up in the extensive work of Schmitt (Reference 1) on $\mathrm{DSB} / \mathrm{FM}$. The tape flutter spectrum measured in his work is given in Reference 2. It apparently caused no trouble. It resembles closely the measured spectrum from which models A1 and A2 were drawn. Optimization of the PLL depends upon a compromise between flutter and noise, taking into account the range of data signals and carrier/noise ratio to be accommodated. At present it appears that the figure of $150 \mathrm{~Hz}$ 
TAELE $\quad 1.4-4$

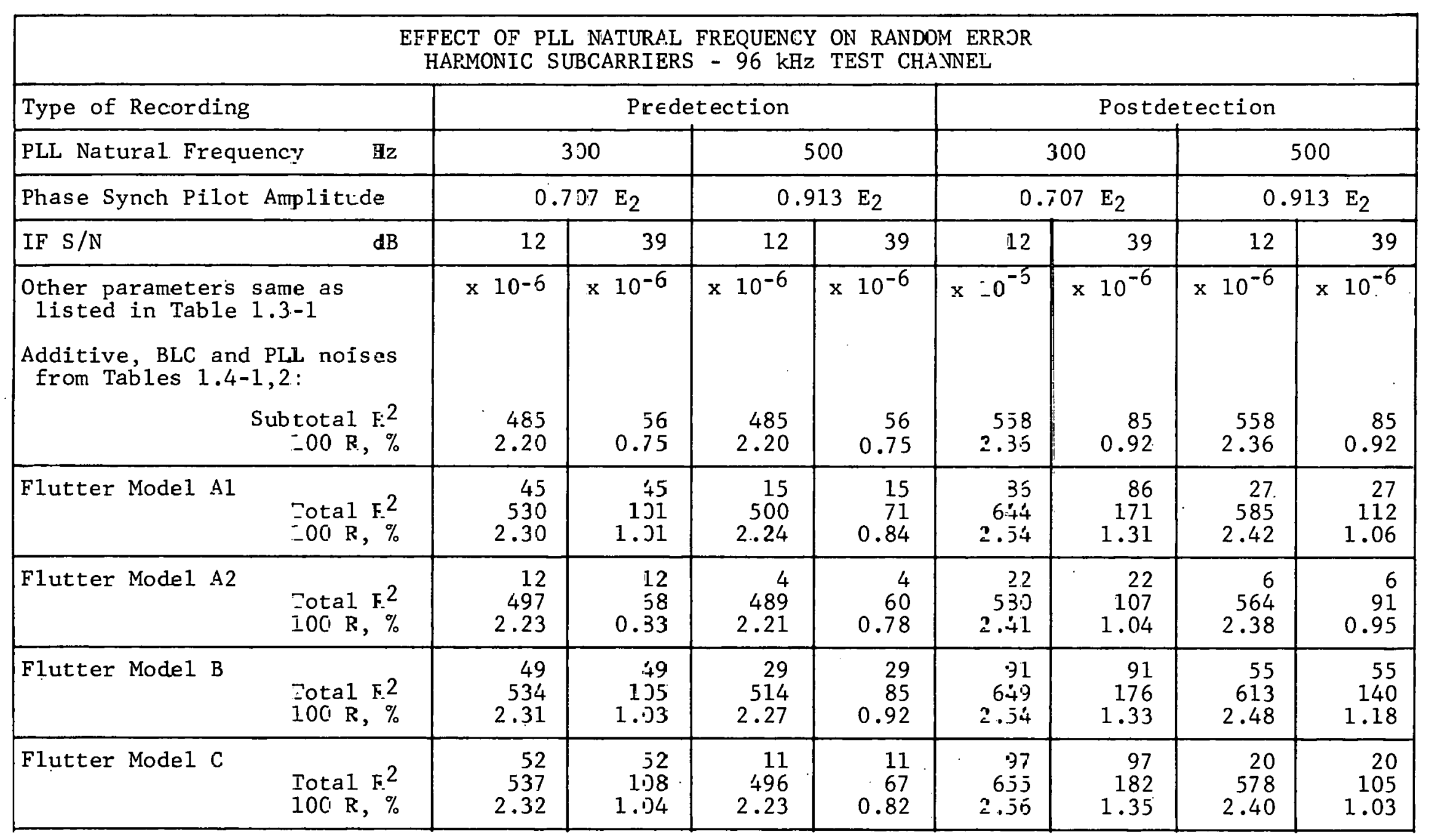




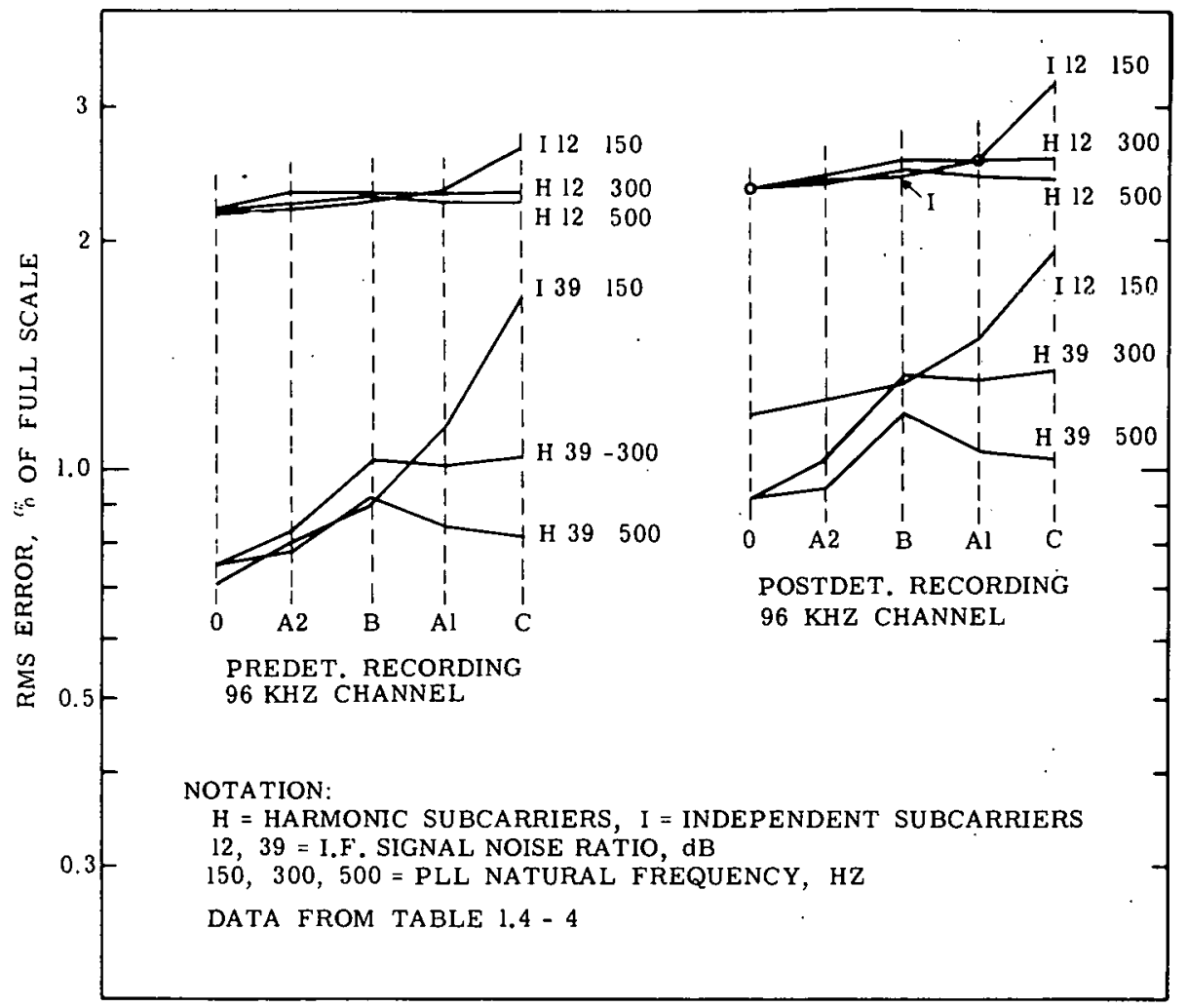

Figure 1.4-4 Effect of PLL natural frequency on random error-harmonious subcarriers

for $f_{n}$ (Reference 2 lists $160 \mathrm{~Hz}$ ) is a reasonable choice. Another way to go is to preemphasize the transmitter modulation for the high channels and then to increase $f_{n}$ if needed to track some tape flutter spectra which are more severe than the A models. This problem was not tackled in the present study.

In order to get an idea. of the utility of the error prediction process reported here, a comparison is made with results obtained by Frank Schmitt of Lockheed at White Sands Missile Range (Reference 1, p. 114). His results deal with a ten-channel system using the channel input voltages as in Table 1.7-1. The independent subcarrier system of that table has twenty channels, so many parameters are different between the two cases. Schmitt had a $3 \mathrm{kHz}$ pilot, we have $1.5 \mathrm{kHz}$. Schmitt had $2 \mathrm{kHz}$ data channels, we have $1 \mathrm{kHz}$. The total transmitter deviation was about the same, being $78 \mathrm{kHz}$ for the Schmitt data. If the noise and distortion models were correct the additive noise percent error would be about the same in both cases, because the transmitter deviation due to each channel would be about $3 \mathrm{~dB}$ greater in the tenchannel case than for twenty channels, making up for the increase in 
bandwidth. The $88 \mathrm{kHz}$ calculations are for the same multiplex as in Tables $1.4-1$ and $1.4-2$ but are not tabulated therein.

Figure 1.4-5 displays the random errors for the two cases using predetection recording. Data is plotted with zero data voltage and with nominal data voltage to show the effects of additive noises and of multiplicative noises. The qualitative behaviour of the two sets is similar but there are significant differences in the size of the effects. A curve taken with the radio link alone (no magnetic tape link) is shown for comparison with the calculated zern-data line. They have the same slope with respect to channel location in the baseband with about $3 \mathrm{~dB}$ ditference in level. This is reasonable agreement rnnsidering that Schmitt's link distortion (Figure 2.2-2) was lower than that used in the model of Figure 2.2-5.

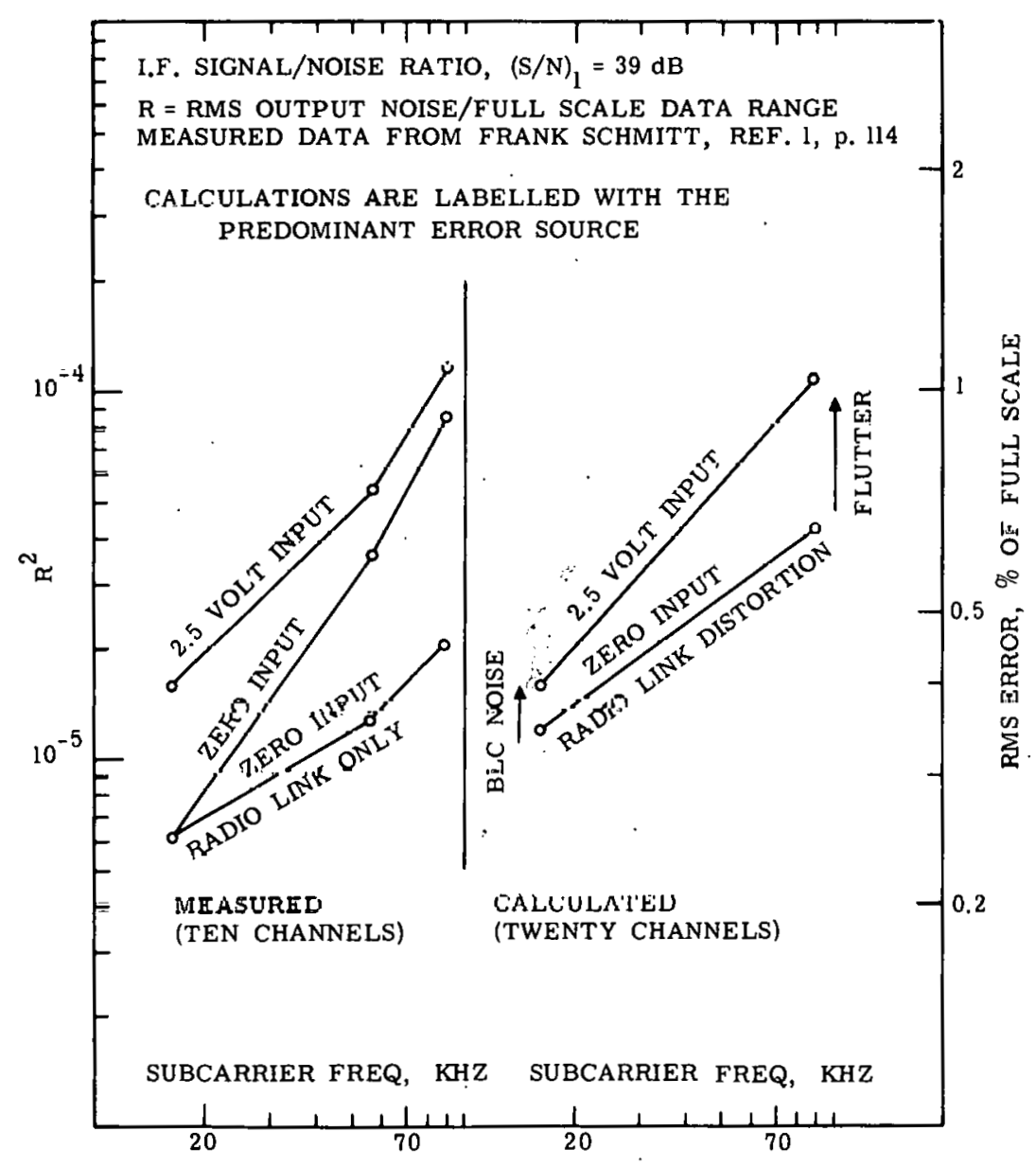

Figure 1.4-5 Test channel output errors with predetection recording independent subcarriers flutter Model $A 1, \theta_{a}=0.1$ radian 
The additive noise with the predetection tape link is appreciably higher than the calculated level at the higher subcarrier channels. The calculations are based on notch noise test data including Schmitt's tape link data (Figure 2.3-2 and 2.3-3), which indicated that the noise and distortion due to the tape link were less than our radio link distortion.

When the data input is set at 2.5 volts dc the multiplicative noise effects are apparent. The flutter effect on $16 \mathrm{kHz}$ is undoubtedly smal1, so the measured increase is attributed to BLC noise modulation of the data output. The measured data for $16 \mathrm{kHz}$ shows 16 microunits compared to 4 microunits for the calculated model. At $88 \mathrm{kHz}$ the increase in the measured output error with data voltage was 30 microunits mean square, of which 16 can be attributed to BLC noise, leaving 14 due to flutter and PLL noise error. Table 1.4-1 shows the expected PLL noise to be negligible, leaving 14 due to flutter, compared to 64 using flutter model Al with 5.7 degrees for the steady phase error. The difference (about $6 \mathrm{~dB}$ ) is about the same as the difference between flutter models $A 1$ and $A 2$. Therefore it appears that the combination of the mode 1 and the phase shift is pessimistic for the conditions achieved in Schmitt's system, whereas the BLC noise due to the models appears too low, as does the predetection additive noise. By coincidence the overall error with 2.5 volt input to the test channel is closely the same in both the measurements and calculations, ranging from 0.6 to 1.0 percent in going from 16 to $88 \mathrm{kHz}$.

Figure 1.4-6 shows the same type of data for a system using postdetection recording. Here again we find the measured additive noise contribution due to recording to be larger than expected from the models based on notch noise tests, running from 20 to 50 microunits (mean square) across the baseband. We calculate about 90 microunits of noise at 2.5 volts due to the BLC, compared to a measured increase of 250 microunits, a ratio of $4.4 \mathrm{~dB}$. A decrease would be expected, rather than an increase, because of the relative location of the two pilots in the postdetection recording noise spectrum model. The agreement between the trends of the measurements and the calculations is not particularly gratifying, considering that data on the same links were used in selecting the noise and distortion models. 


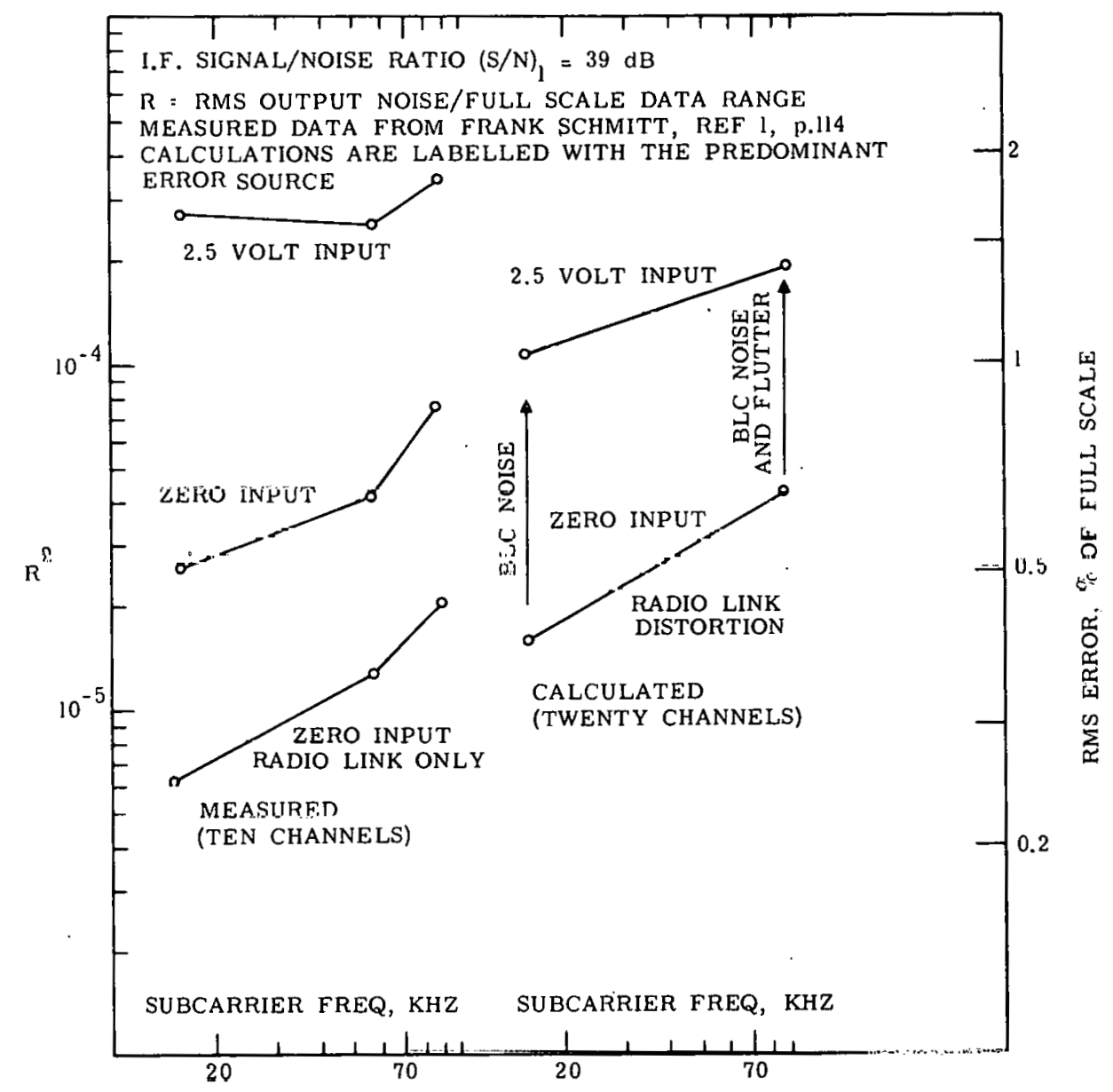

Figure 1.4-6 Test channel output errors w1th postdetection. recurding independent subcarriers, flutter Model $\mathrm{A} 1, \theta_{\mathrm{a}}=0.1$ radian

\subsection{ERROR CAUSES}

\subsection{Distortion of Baseband Signals}

W. E. Thompson (Reference 3) has developed a series solution for the autocorrelation function of the output of a nonlinear system when the input is Gaussian random noise. The series is composed of powers of the normalized autocorrelation function of the input, which when transformed to obtain the output spectrum gives a series in selfconvolutions of the normalized input signal spectrum, Equation 2.1-3. The coefficients, Equation 2.1-4, are taken from Reference 3.

The output, $y$, of a nonlinear device with input, $x$, is expressed as a power series:

$$
y=a_{0}+a_{1} x+a_{2} x^{2}+a_{3} x^{3}+\cdots \cdot
$$


The input, $x(t)$, is assumed to be Gaussian random noise with rms value $X$, and two-sided power spectrum $S_{X}(f)$, whose normalized version is defined by:

$$
S_{x}(f)=x^{2} s_{x}(f)
$$

The normalized spectrum, $s_{X}(f)$, has the same frequency extent as $S_{X}$, but unity mean square integral. The spectrum of the output, $y(t)$, is $s_{y}(f)$, given by:

$$
\begin{aligned}
s_{y}(f)= & F_{1}(X) s_{x}(f)+2 ! F_{2}(X) s_{x}^{(2)}+3 ! F_{3}(X) s_{x}^{(3)}+\cdots \\
& +n ! F_{n}(X) s_{x}(n)+
\end{aligned}
$$

ignoring any $d c$ component in the output. The factors, $F_{n}$, are functions of the rms level of the input and determine the level of the $n$th order distortion spectrum. The factors, $s_{x}(n)$, are $n^{\text {th }}$ order self-convolutions of the normalized input spectrum and determine the shape of the $n^{\text {th }}$ order distortion spectrum. The factors, $F_{n}$, as derived by Thompson are:

$$
F_{n}=\left[\sum_{j=0}^{\infty} \frac{(n+2 j) !}{2^{j} j ! n !} a_{n+2 j} x^{n+2 j}\right]^{2}
$$

From the expression for $\mathrm{F}_{\mathrm{n}}$ it is seen that all even powers in the power series contribute the second order distortion, and all odd powers contribute to the third order, the relative contribution of higher powers rising with the input rms, $X$. In some cases either the second or third power contribution may predominate, as may be noted from measurement of output distortion versus input level. In the case of the second power term, the contribution to the output distortion to signal power ratio is :

$$
\frac{s_{y 2}}{a_{1}{ }^{2} s_{x}}=2\left(\frac{a_{2}}{a_{1}}\right)^{2} x^{2} \frac{s_{x}(2)}{s_{x}} .
$$

where $S_{y 2}$ is the second-order distortion spectrum and $a_{1}{ }^{2} s_{x}$ is the output signal in the absence of distortion. The third-order distortion to signal ratio due to third pwoer term is:

$$
\frac{\mathrm{s}_{\mathrm{y} 3}}{\mathrm{a}_{1}{ }^{2} \mathrm{~s}_{\mathrm{x}}}=6\left(\frac{\mathrm{a}_{3}}{\mathrm{a}_{1}}\right)^{2} \mathrm{x}^{4} \frac{\mathrm{s}_{\mathrm{x}}(3)}{\mathrm{s}_{\mathrm{x}}}
$$

where $\mathrm{S}_{\mathrm{y} 3}$ is the third-order distortion spectrum. 
For a flat input signal spectrum whose one-sided version extends from zero to $\mathrm{fb}_{\mathrm{b}}$, the ratio $\mathrm{s}_{\mathrm{x}}(2) / \mathrm{s}_{\mathrm{x}}$ and $\mathrm{s}_{\mathrm{x}}{ }^{(3)} / \mathrm{s}_{\mathrm{x}}$ are easily calculated and are shown in Figure 2.1-1 as a function of frequency within the band of the input. The relative flatness of these curves, characteristic of static distortion, is also characteristic of higher-order self-convolutions.

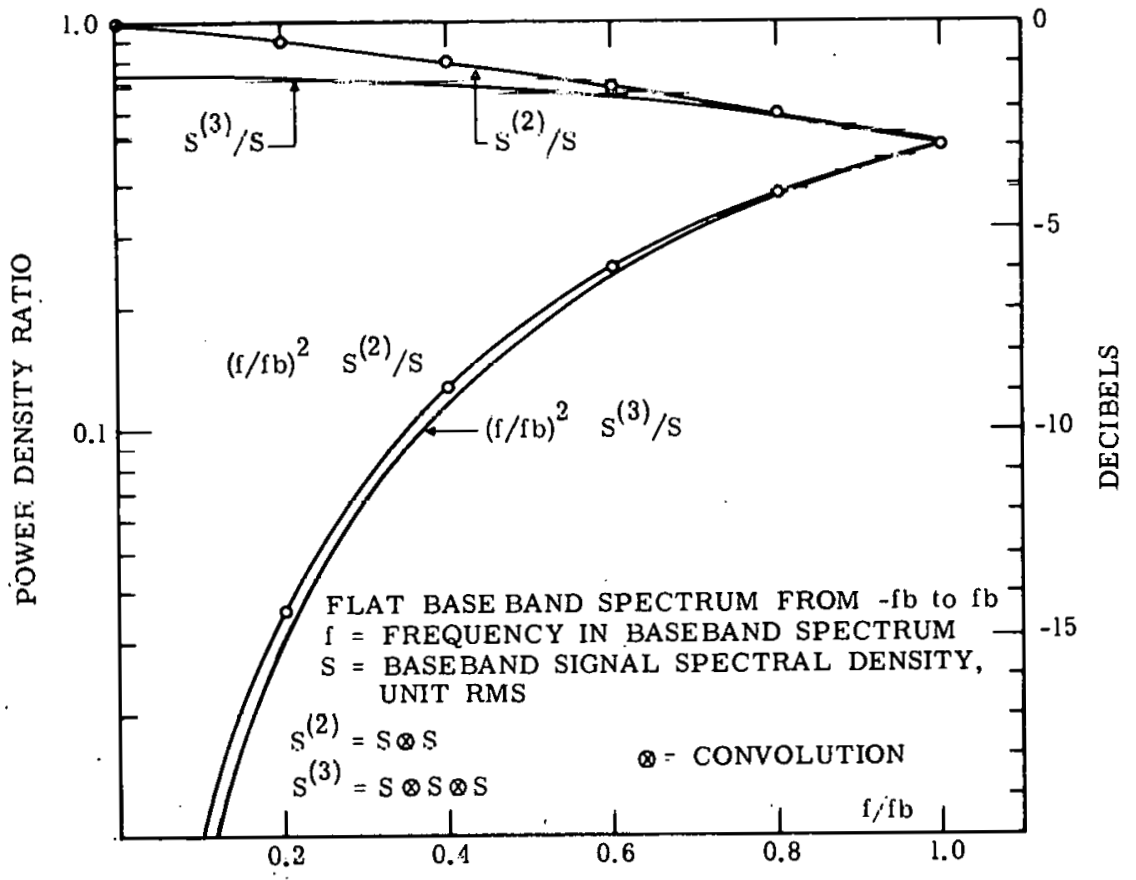

Figure 2.1-1 Distortion spectra1 distribution

Direct measurement of the coefficients of the power series representation is somewhat tricky, particularly for the higher orders. It is possible to find the coefficients from harmonic distortion of sinusoidal signals, however, when the nonlinearity is gentle, in the sense that the higher-power terms don't get overly strong in the interesting range of input levels. The $n^{\text {th }}$ harmonic level is due to the $(n+2 j)-$ power terms as in the case of the random signal. Distortion ratios are calculated for the various powers using trignometric identities. The second-power coefficient from second harmonic measurement is:

$$
\left(a_{2} / a_{1}\right)=2 r_{2} / A
$$


where $A$ is the peak amplitude of the sinewave input and $r_{2}$ the ratio of second harmonic to fundamental voltage in the output. If this ratio stays fairly constant as $A$ is varied from zero up to the range of interest, this indicates that the second power contribution predominates among the even powers. Similarly, the third power coefficient may be deduced from:

$$
\left(a_{3} / a_{1}\right)=4 r_{3} / A^{2}
$$

Again, the predominance of the third-power contribution will be indicated by relative constancy of this ratio as $A$ is raised from zero.

In the event a piece of equipment exhibits strong higher-order contributions, attemps to express the system behaviour analytically become difficult to calculate, particularly when it is recognized that there are also frequency-sensitive nonlinear effects with various powers, orders, etc. Thompson (Reference 3) also developed a means of expressing harsh nonlinearities as a series of Hermite polynomials, the coefficients of which can be derived by numerical integration from a known transfer function, but this did not appear applicable to the data available to this work.

Ghais, Ferrari, and Boardman (Reference 4) have treated FM distortion due to filters used in receiver IF amplifiers, both analytically and experimentally. The analytical work starts with Thompson's series for static distortion, applies it to the phase vs frequency characteristic of the IF filter, and then multiplies the resulting phase-modulation distortion by $\mathrm{f}^{2}$ to obtain the frequency-modulation distortion. The use of the steady-state phase characteristic of the filter as the nonlinearity model appeared justified by measurements of distortion, over the range of parameters tested. The following discussion gives some insight into the derivation.

The filter phase characteristic is expressed as:

$$
y=x+b_{0}+b_{1} w_{1}+b_{2} w_{1}^{2}+b_{3} w_{1}^{3}+. .
$$

where $\mathrm{x}$ is the input phase modulation, $\mathrm{y}$ is the output phase modulation, and $\omega_{1}$ is the input frequency modulation, $\mathrm{dx} / \mathrm{dt}$. As defined here the phases are in radians, the frequency in radians/second. The terms in $b_{0}$ and $b_{1}$ represent constant phase shift and constant delay, respectively, but not distortion. The terms in $b_{2}$ and above contribute to phase-error 
in the same way as for the static case discussed previously. The receiver demodulator is sensitive to phase rate rather than phase, so each distortion spectral component is multiplied by $(2 \pi \mathrm{f})^{2}$ before comparing it with the signal spectral density at the same frequency. As a result, the distortion-to-signal ratio is much more sensitive to location in the baseband than in the case of static distortion.

The power spectrum of the frequency-modulating signal, $\omega_{1}$, is $S_{w}(f)$ and its normalized version is $s_{\mathrm{W}}(f)$. The rms frequency modulation, in radians/second, is $\mathrm{W}$. Then:

$$
S_{\mathrm{W}}(f)=W^{2} s_{\overline{\mathrm{w}}}(f)
$$

As in the case of static nonlinearity, the phase-modulation distortion spectrum is given by:

$$
s_{y}(f)=\sum_{m=2}^{\infty} n ! F_{n}(W) s_{w}(n)
$$

where

$$
F_{n}(w)=\left[\sum_{j=0}^{\infty} \frac{(n+2 j) !}{2 \cdot \dot{i}_{j} ! n !} b_{n+2 j} w^{n+2 j}\right]^{2}
$$

The FM distortion spectrum resulting from the PM spectrum, Equation $2.1-11$, is :

$$
S_{z}(f)=\omega^{2} S_{y}(f)
$$

and the distortion/signal power density ratio:

$$
\frac{3_{z}(I)}{S_{w}(f)}=\sum_{n=2}^{\infty} \omega^{2} n: \frac{F_{n}(W) v_{w}(n)(f)}{W^{2} s_{w}(f)}
$$

For IF filters which arc oymmetrical about the carrier frequency, the third-order distortion spectrum is of main interest, with the fifth order growing as the modulation level, $\mathrm{W}$, is increased. The factor, $\mathrm{F}_{3}$, associated with the third order spectrum is:

$$
F_{3}(w)=w^{6}\left[b_{3}+10 b_{5} w^{2}+105 b_{7} w^{4}+\cdot \cdot\right]^{2}
$$


From Equations 2.1-14 and 2.1-15, the third-order distortion/signal power ratio is:

$\frac{S_{z 3}}{S_{w}}=6(2 \pi)^{6} f^{2} F_{d}{ }^{4} \frac{s_{w}^{(3)}}{s_{w}}\left[b_{3}+10 b_{5}\left(2 \pi F_{d}\right)^{2}+105 b_{7}\left(2 \pi F_{d}\right)^{4}.\right]$

where $W$ has been replaced by $2 F_{d}$ and $F_{d}$ is the rms deviation in Hertz . Normalizing Equation 2.1-16 with respect to the IF bandwidth, $\mathrm{B}_{1}$, permits use of previously calculated results (References 4 and 5).

$$
\frac{\mathrm{S}_{\mathrm{z}}}{\mathrm{S}_{\mathrm{w}}}=\underbrace{\left(3: 2^{6}\right)}_{\mathrm{K}} \cdot \underbrace{\left(\frac{\mathrm{f}_{\mathrm{b}}}{\mathrm{B}_{1}}\right)^{2}\left(\frac{\mathrm{F}_{\mathrm{d}}}{\mathrm{B}_{1}}\right)^{4}}_{\mathrm{G}_{1}} \underbrace{\left(\frac{\mathrm{f}}{\mathrm{f}_{\mathrm{b}}}\right)^{2} \cdot \frac{\mathrm{s}_{\mathrm{w}}{ }^{(3)}}{\mathrm{s}_{\mathrm{w}}}}_{\mathrm{G}_{2}} \underbrace{\left(\frac{2 \pi \mathrm{B}_{1}}{2}\right)^{2}\left[\mathrm{~b}_{3}+\ldots \cdot \cdot\right]}_{\mathrm{G}_{3}}
$$

The factor $\mathrm{K}$ is $25.8 \mathrm{~dB}$. The factor $\mathrm{G}_{2}$ is shown in Figure 2.1-1. The factor $G_{3}$, when $F_{d} / B_{1}$ is 0.16 or less, is about $-4 \mathrm{~dB}$ for filters similar to the high-order Butterworth. For linear-phase filters, $G_{3}$ is about $-21 \mathrm{~dB}$ based on measurements. For the theoretical Bessel filter, $\mathrm{G}_{3}$ is much lower but rising rapidly for $F_{\mathrm{d}} / B_{1}$ larger than 0.15 . The factor $G_{1}$ is plotted in Figure 2.1-2.

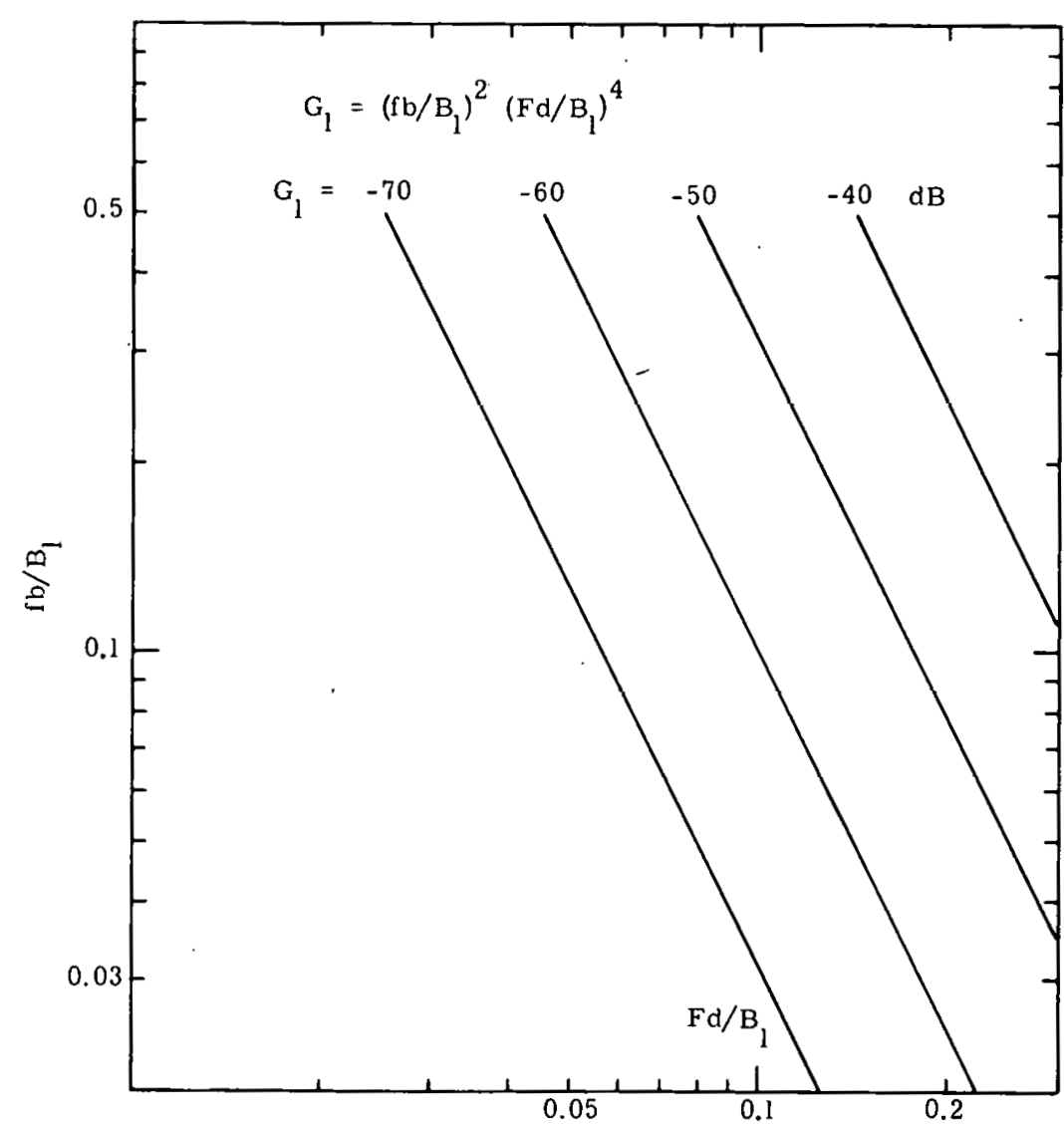

Figure 2.1-2

IF filter distortion tactors 
This discussion has oversimplified the FM distortion relationships, but within a useful range of parameters, experiments have followed Equation 2.1-17 within a $\mathrm{dB}$ or so, as discussed in Reference 5. 
Symbols used in Section 2.1

$a_{1}, a_{2}, \ldots a_{n}$ Coefficients of power series for static nonlinear device

$\mathrm{b}_{1}, \mathrm{~b}_{2}, \ldots \mathrm{b}_{\mathrm{n}}$. Coefficients of power series for non1inear phase characteristic

$\mathrm{B}_{1}$

IF filter bandwidth, Hertz

$\mathrm{F}_{1}, \mathrm{~F}_{2}, \ldots \mathrm{F}_{\mathrm{n}}$

Coefficients of series for spectrum of output of nonlinear device

$F_{d}$

rms carrier deviation, Hertz

Factors in approximate expression for IF filter distortion

Factor in approxiamte expression for IF filter distortion

$r_{2}$

Ratio of second harmonic to fundamental frequency voltage

Ratio of third harmonic to fundamental frequency voltage

Power spectrum of $x(t)$

Normalized version of $\mathrm{S}_{\mathrm{x}}$

Power spectrum of $y(t)$

$2.1-3$

Power spectrum of PM distortion

$n^{\text {th }}$ order self-convolution of $s_{x}$

$2 \cdot 1-3$

Terms in series for $\mathrm{S}_{\mathrm{y}}$

$2.1-5$

$2.1-6$

$\mathrm{S}_{\mathrm{W}}$

$\mathrm{s}_{\mathrm{w}}$

$s_{w}(n)$

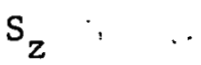

W

$\mathbf{x}$

$\mathrm{X}$

$\mathrm{y}$

$\omega_{1}$
Power spectrum of carrier deviation, $\omega_{1}(t)$

$2.1-10$

Normalized version of $\mathrm{S}_{\mathrm{w}}$

$n^{\text {th }}$ order self-convolution of $s_{W}$

Power spectrum of FM at IF filter output

rms value of carrier deviation, $\omega_{1}(t)$, radian/ second

$2.1-10$

$2.1-11$

$2.1-13$

Inpul to stalic numlinear device

$2.1-10$

2.1-1

rms value of $x(t)$

$2.1-2$

Output of nonlinear device

$2.1-1$

Instantaneous frequency deviation of input to IF filter 


\subsection{Radio Link Noise}

For low carrier-to-noise ratios (10 to $20 \mathrm{~dB}$, say) the main component of receiver output noise is due to thermal noise entering the antenna and generated in the receiver front end. The noise power density at the input is $F_{n} \mathrm{KT}$ when the receiver is operating from a signal generator in the laboratory. $F_{n}$ is the receiver noise factor, $K$ is Boltzmann's constant, and $\mathrm{T}$ is "room temperature," about 290 degrees Kelvin. This noise power density, $p_{1}$, when added to the input signal, of power $P_{1}$, causes the resultant input to the FM demodu1ator to be phase-modulated with a modulation density $q_{2}$ given hy:

$$
\mathrm{q}_{2}=\frac{\mathrm{P}_{1}}{\mathrm{P}_{1}}=\frac{\mathrm{F}_{\mathrm{n}} \mathrm{KT}}{\mathrm{P}_{1}}=\frac{1}{\mathrm{~B}_{1}(\mathrm{~S} / \mathrm{N})_{1}^{2}} \quad \text { Radian }{ }^{2} / \text { Hertz }
$$

where $(S / N)_{1}$ is the RMS signal/noise ratio in the IF bandwidth, $B_{1}$. The phase modulation produces a frequency-modulation density proportional to $\mathrm{f}^{2}$ where $\mathrm{f}$ is a frequency in the baseband at the receiver output. The receiver output noise power (or mean square) density, $\mathrm{p}_{2}$, is:

$$
\mathrm{p}_{2}=\mathrm{f}^{2} \frac{\mathrm{P}_{1}}{\mathrm{P}_{1}}=\frac{\mathrm{f}^{2}}{\mathrm{~B}_{1}(\mathrm{~S} / \mathrm{N})_{1}^{2}} \quad \text { Hertz }{ }^{2} / \text { Hertz }
$$

The noise density, in mean square equivalent frequency modulation of the carrier, may also be expressed in volt $t^{2} /$ Hertz by multiplying $p_{2}$ by the square of the receiver modulation sensitivity. This factor depends upon several gains, including the video gain control setting, and does not fundamentally affect the overall results.

Equation (2.2-2) loses accuracy in the lower portinn of the baseband at lower signal-to-noise ratios. The low baseband noise is essentlally flat (independent of $f$ ) with a density that depends on the type and intensity of modulation as well as the signal-to-noise ratio. For a flat (nonpreemphasized) haseband signal spectrum the low basuband nolse level is not significant. If preemphasis is used, the low-frequency density is used to select the frequency at which the preemphasis network corners. The relationships are discussed in Reference 6 .

There is limited information on the noise at low frequencies under various modulation conditions. Departure of notch-noise test data (Reference 1, p. 38) from Equation 2.2-2 indicates that the intercept of the flat noise spectrum with the $\mathrm{f}^{2}$ spectrum occurs at about $10 \mathrm{kHz}$ with flat 
random modulation, $78 \mathrm{kHz}$ rms deviation, with $(\mathrm{S} / \mathrm{N})_{1}$ of $12 \mathrm{~dB}$ in 460 $\mathrm{kHz}$ IF bandwidth. In Figure 2.2-1 curve $\mathrm{A}$ is the measured data, curve $B$ the calculated data for these conditions. Therefore, the noise density for low-frequency pilot signal locations. may be estimated from the $10 \mathrm{kHz}$ value from Equation 2.2-2 under these conditions..

As the carrier-to-noise ratio is rasied, the noise density due to front-end noise decreases. The low frequency noise decreases more rapidly, so that the corner moves to lower frequencies. Other noise effects are observed, however, which have not been well. explained and are referred to as residual noise, incidental FM, etc. Measurements at $(\mathrm{S} / \mathrm{N})_{1}$ of $39 \mathrm{~dB}$ are shown as curve $\mathrm{C}$, with the calculated level as curve $\mathrm{D}$. The measurements were taken with an unmodulated carrier, in order to eliminate noise due to distortion, which is, much higher. . The noise shown at $14 \mathrm{kHz}$ can be extrapolated to lower frequencies to obtain a model random component. It is likely that several spectrum lines

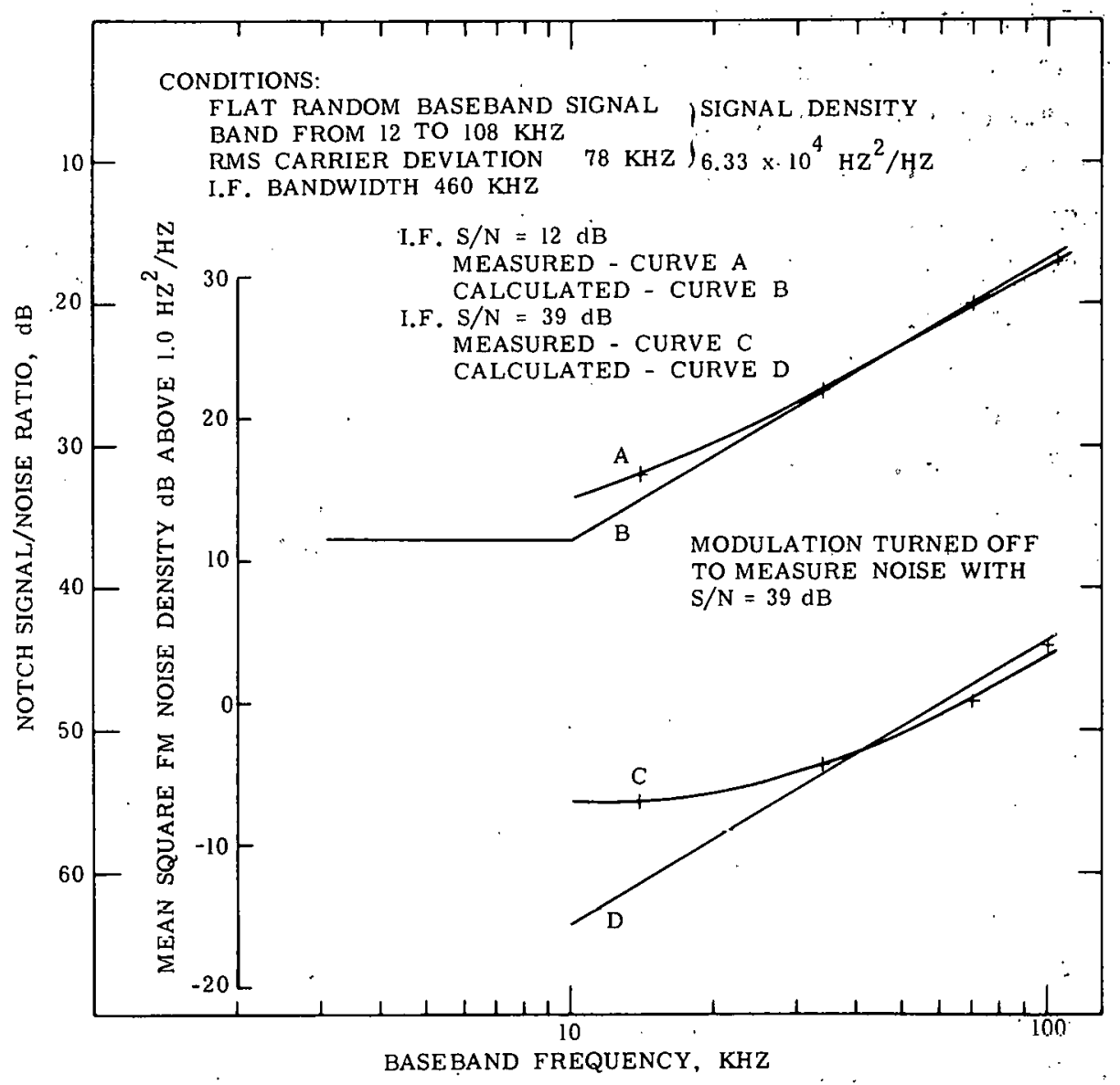

Figure 2.2-1 Radio link baseband noise density 
will stick up at harmonics of the prime power frequencies of the transmitter and receiver in addition. There may also be significant $1 / f$ noise at low frequencies due to semiconductor devices in the video stages. There has recently been interest in random PM caused by varactor multiplier in highly stable signal sources, so there is a possibility that early stages of the receiver, including the local oscillator, IF, and limiter, contribute some modulation even with strong signals. It appears that the low-frequency noise level will be well below the distortion level, however, and no effort was made to get a good picture of residual noise.spectrum below $10 \mathrm{kHz}$.

Thie nutch nulse cest results in ratios which are essentially the ratio of random signal power density to noise power density at a chosen frequency in the baseband. A multiplex FDM channel using the same link will exhibit a different signal-to-noise ratio from the notch noise power ratio, for several reasons. In the case of noise inherent in the link, not caused by the signal, the conversion to absolute units (rather than ratio of noise to signal) is straightforward. To get the noise density it is necessary only to divide the signal power density by the signalto-noise power ratio. To get the signal density, at a point in the system where the signal spectrum is flat, it is only necessary to divide the mean square signal level by the signal bandwidth. In Figure 2.2-1 the mean square signal level is $(78,000)^{2} \mathrm{Hertz}^{2}$, and the signal bandwidth (108,000-12,000) Hertz, giving a signal density of $63,000 \mathrm{Hertz}^{2} /$

Hertz, or $48 \mathrm{~dB}$ above 1 Hertz ${ }^{2} /$ Hertz. Then when the signal-to-noise ratio is $48 \mathrm{~dB}$, the noise density is 1 Hertz ${ }^{2} / \mathrm{Hertz}$. From this calculation is produced the scale of absolute FM noise density shown on Figure $2.2-1$.

\section{Radio Link Distortion}

The radio link has several elements which contribute to the static nonlinear transfer characteristic, including the transmitter modulator, the receiver demodulator, and the baseband amplifiers. In addition there is frequency modulation distortion caused by the receiver IF filter. These effects are discussed analytically in Section 3.1. Distortion measurements on telemetry radio equipment show a wide spread in distortion levels, so a distortion model for error estimation will be a matter of judgment which will not be justifiable in all circumstances. 
The total distortion consists of various types and orders. The measurement data is useful in judging which of these should be included in the model. The variation in distortion level with baseband frequency and with transmitter deviation level are helpful in this regard. Figure 2.2-2 shows notch noise test data for several radio links (in the laboratory) as a function of notch frequency. Figures 2.2-3 and 2.2-4 show the variation in notch signal-to-noise ratio with respect to transmitter deviation at high and low baseband frequencies, respectively.

The variation with frequency distinguishes between static distortion and IF filter distortion, the former being relatively flat with frequency and the latter rising rough $1 y$ as $\mathrm{f}^{2}$ in mean square (power) density. Curve A, Figure 2.2-2 shows no sign of filter distortion, being taken with a $3.3 \mathrm{MHz}$ IF bandwidth, $108 \mathrm{kHz}$ flat random baseband signal, and $85 \mathrm{kHz}$ rms deviation. The signal generator was an S-band Klystron with repeller modulation, thought to be very linear. Curves A in Figures 2.2-3 and 2.2-4 show the signal-to-noise ratio to be relatively flat with modulation variation, so this noise is not characteristic of link distortion. Curve $B$ in the three figures is for the same signal generator and receiver with a $500 \mathrm{kHz}$ IF filter and demodulator

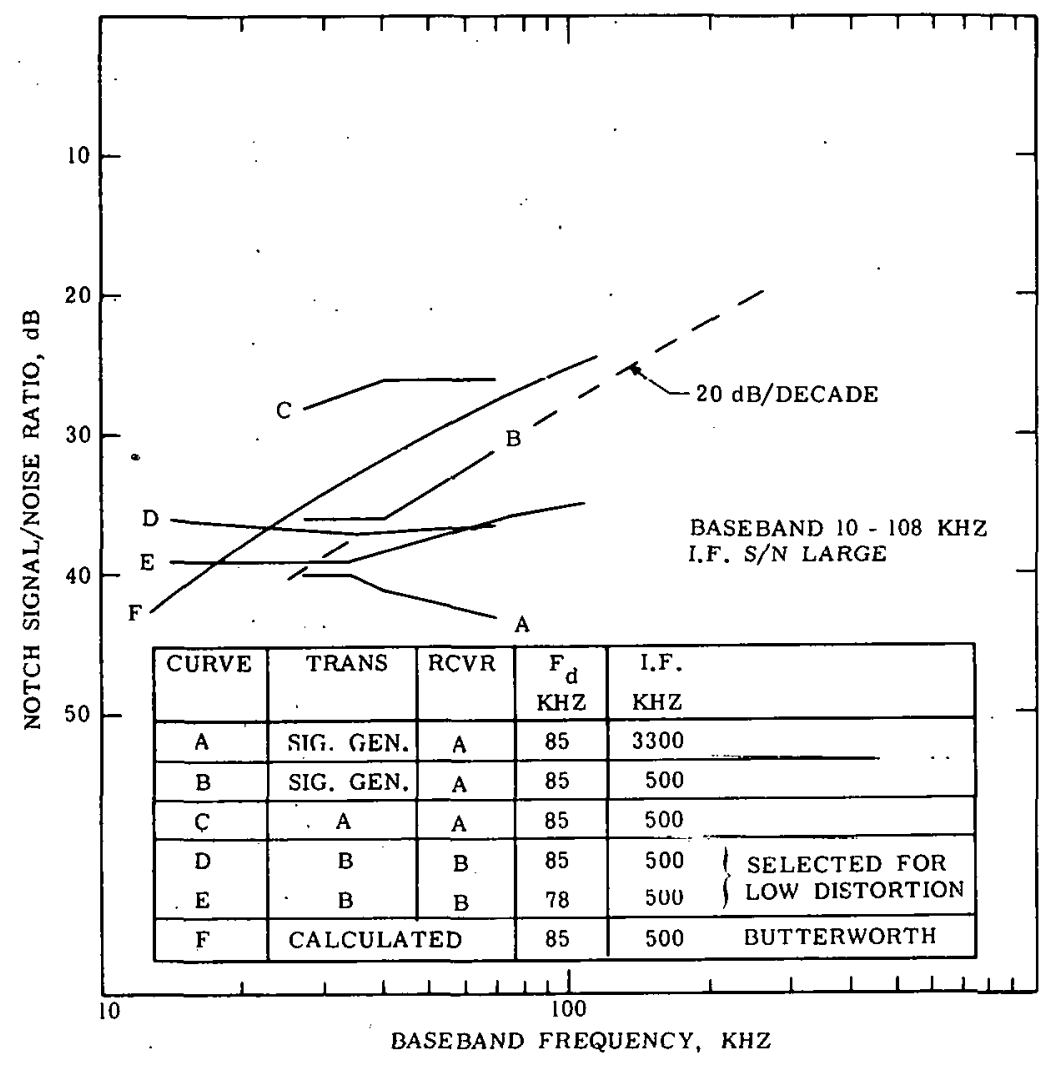

Figure 2.2-2

Radio link notch noise tests 


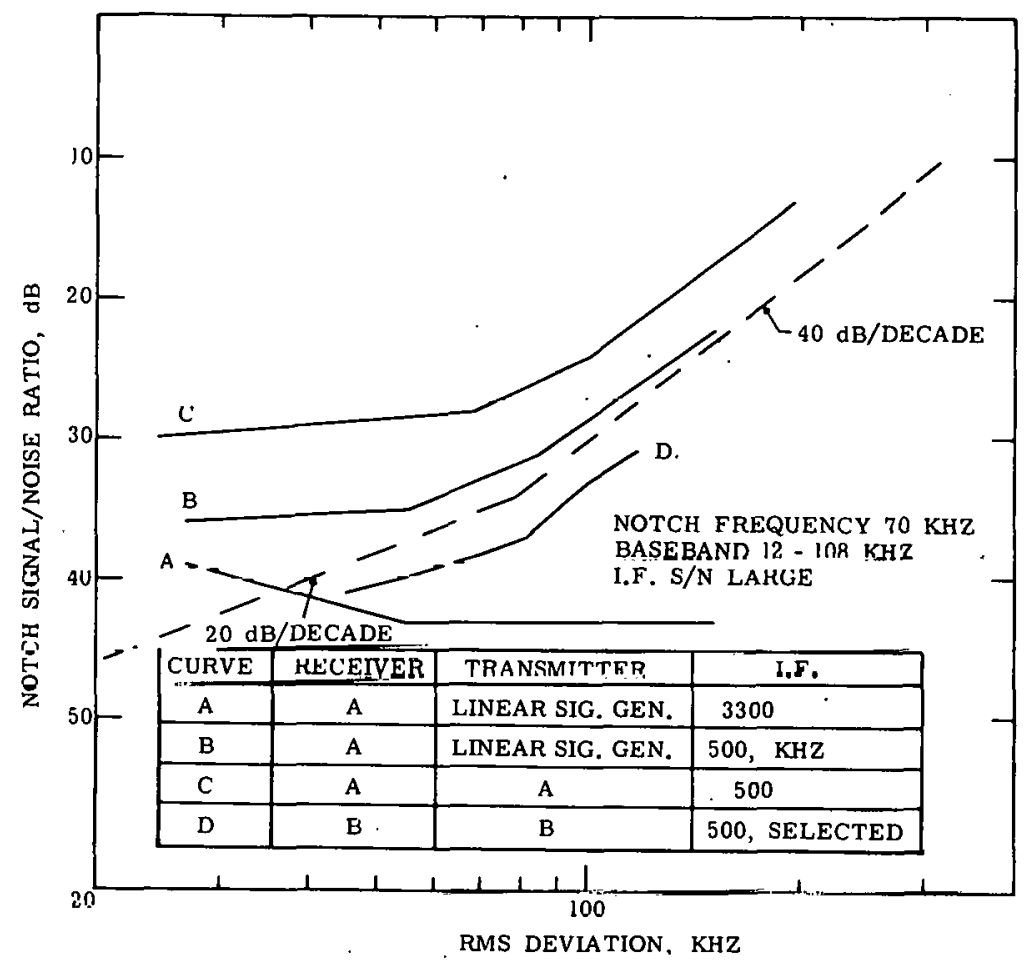

Figure 2.2-3 Radio link notch noise tests

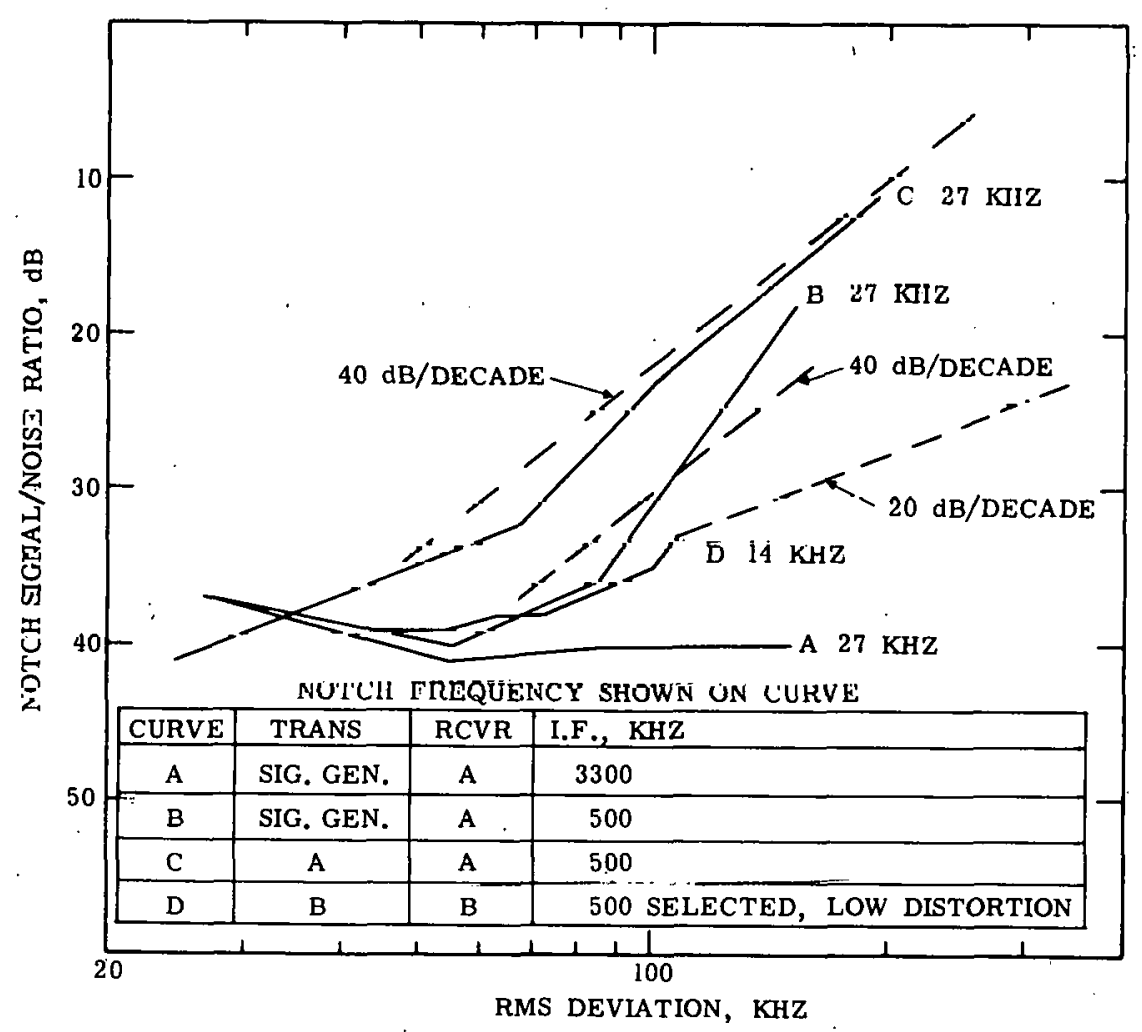

Figure 2.2-4 Radio link notch noise tests 
in the receiver. There is an increase in noise-to-signal power ratio with frequency between 40 and $70 \mathrm{kHz}$ proportional to $\mathrm{f}^{2}$. Extending this to $27 \mathrm{kHz}$ gives a noise level equal to Curve $\mathrm{A}$ at this point. Adding this to Curve $A$ gives $3 \mathrm{~dB}$ increase at $27 \mathrm{kHz}$ compared to the $4 \mathrm{~dB}$ increase noted. Hence we find roughly the additional noise expected from IF filter distortion. The $40 \mathrm{~dB} /$ decade slope of curve $B$ in Figure 2.2-3 is characteristic of third-power nonlinearity in the filter phase vs frequency characteristic. At $27 \mathrm{kHz}$, Figure 2.2-4 the agreement with $\mathrm{F}_{\mathrm{d}}^{4}$ slope is not good.

Curves $C$ in these three figures are taken with the same receiver, $500 \mathrm{kHz} \mathrm{IF}$, and a transmitter whose distortion is greater than that of the Klystron signal generator. The noise does not rise with frequency, and the noise-to-signal power ratio increases at $40 \mathrm{~dB} /$ decade with modulation level, hence it appears that this is mostly third-power static distortion. Curves A, B, and C are based on unpublished data supplied by C. S. Johnson of Sandia Laboratories, Albuquerque.

Curves $\mathrm{D}$ and $\mathrm{E}$ are taken from pages 39 and 38, respectively, of Reference 1. The receiver IF filter bandwidth is about $500 \mathrm{kHz}$, and the filter was selected from stock to have the lowest distortion. The transmitter is thought to have less distortion than several other available models. This combination doesn't exhibit pronounced filter distortion in Figure 2.2-2, but the difference in characteristics between Figure 2.2-3 (40 dB/decade above $80 \mathrm{kHz}$ deviation for $70 \mathrm{kHz}$ baseband location) and Figure 2.2-4 (20 dB/decade for $14 \mathrm{kHz}$ baseband location) indicate that filter distortion would be more pronounced at higher deviations .

Curve $\mathrm{F}$ of Figure 2.2-2 is the estimated signal-to-distortion ratio for a Butterworth filter, using the approximation given in Reference 5 .

Many other notch noise tests are reported in References 7 and 8 . For rms carrier deviations in the neighborhood of $80 \mathrm{kHz}$, using the lowest receiver distortion, the various transmitters produced notch signalto-noise ratios running from 25 to $35 \mathrm{~dB}$ at $40 \mathrm{kHz}$. Curves $\mathrm{D}$ and $\mathrm{E}$ are somewhat better than this, so a level of $35 \mathrm{~dB}$ can be obtained, and will be used in the model for the low-frequency portion of the baseband. For the high-frequency portion, a line with $20 \mathrm{~dB} /$ decade slope, passing through $28 \mathrm{~dB}$ at $100 \mathrm{kHz}$, will be used. The assumed rms carrier deviation 
for these levels will be $80 \mathrm{kHz}$. The signal density will be calculated assuming a $100 \mathrm{kHz}$ random spectrum. From these figures, the signal density is $64,000 \mathrm{Hertz}^{2} / \mathrm{Hertz}$, or $48 \mathrm{~dB}$ above 1.0 . The signal-to-noise ratio of $35 \mathrm{~dB}$ then indicates a distortion density of $13 \mathrm{~dB}$, or $20 \mathrm{Hertz}^{2} /$ Hertz mean square equivalent carrier deviation for the low frequency portion. The rising portion is equivalent to a mean square modulation density of $10^{-8} \mathrm{f}^{2}$. The model spectrum is plotted in Figure 2.2-5. For estimating the effect of changing the rms modulation level, a thirdpower nonlinearity be assumed for the static portion and for the IF filter distortion. In this case the mean square distortion noise density will vary as $F_{d}{ }^{6}$, where $F_{d}$ is the rms transmitter deviation. The sigual power will vary as $F_{d}^{2}$, rcoulting in a signal-co-noise variation as $1 / \mathrm{F}_{\mathrm{d}} 4$.

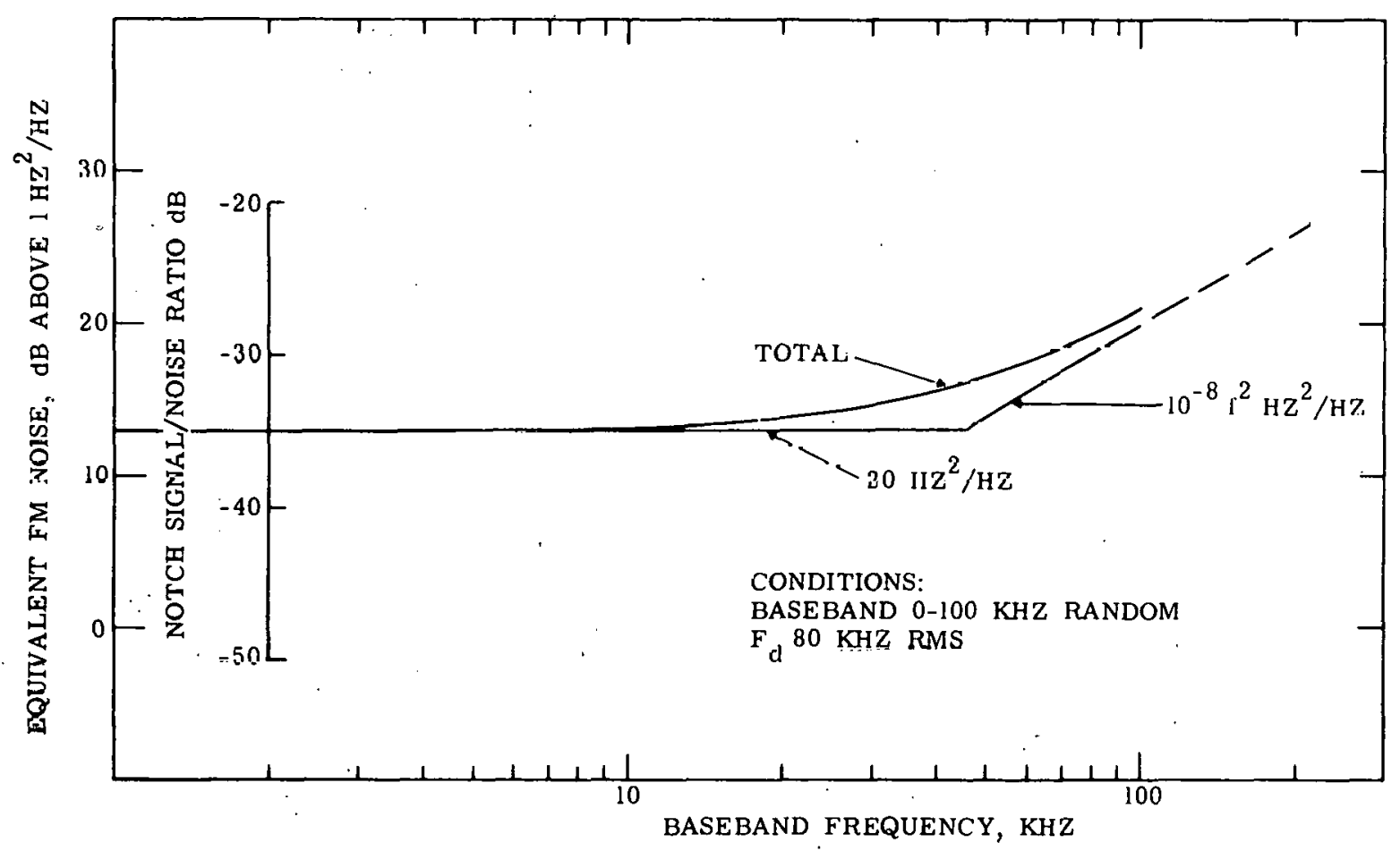

Figure 2.2-5 Radio link distortion model 


\subsection{Tape Link Noise and Distortion \\ Postdetection Tape Link Noise and Distortion}

Reference 1 contains valuable test data on tape recording performance using independent DSB subcarriers. Among the tests were notch noise tests on various magnetic tape recorder/reproducers operating in the postdetection mode, recording the simulated baseband without an RF link. The notch frequencies ranged from 14 to $105 \mathrm{kHz}$, and recording level was changed over a $20 \mathrm{~dB}$ range to determine the best operating level as a compromise between noise and distortion. In order to judge the effect of noise and distortion on pilot signals below $14 \mathrm{kHz}$, the results are examined here with a view to extrapolation. The best performance obtained is recorded in Table II-18, page 46 of Reference 1 .

Absolute recording levels are unknown because the indicator was not calibrated for random signals. It will suffice, however, to use relative record levels, referencing all noise densities to the random signal density. The extrapolation procedure consists of finding the type of intermodulation, the order of nonlinearity, noise contribution, extrapolating them according to individually simple models, and recombining them.

Figure 2.3-1 is a plot of measurements and calculations derived therefrom. Curve $A$ is the notch signal-to-noise ratio at the maximum record level, and is assumed to be due almost entirely to distortion. The slope from 14 to $70 \mathrm{kHz}$ is about right for static distortion. The noise at $105 \mathrm{kHz}$ is lower than the static model would indicate, and this is thought to be because the $105 \mathrm{kHz}$ notch filter is known to have insertion loss extending far from its center frequency, which lowers the overal1 rms level appreciably when the notch filter is inserted, and hence lowers the distortion below that expected with an ideal notch.

Figure IV-26, page 166, of Reference 1 shows the noise-to-signal power ratio to be roughly proportional to signal power in the neighborhood of $0 \mathrm{~dB}$ level. This is characteristic of a square-law nonlinear term, and this will be used as the extrapolation model. This results in calculated curve $C$ in Figure 2.3-1, which is predicted distortion for $-10 \mathrm{~dB}$ record level.

Returning to Figure IV-26 of Reference 1, we see that the noiseto-signal power ratio is inversely proportional to signal power at -20 $\mathrm{dB}$ record level, as expected for playback electronic noise. Curve $B$ of 


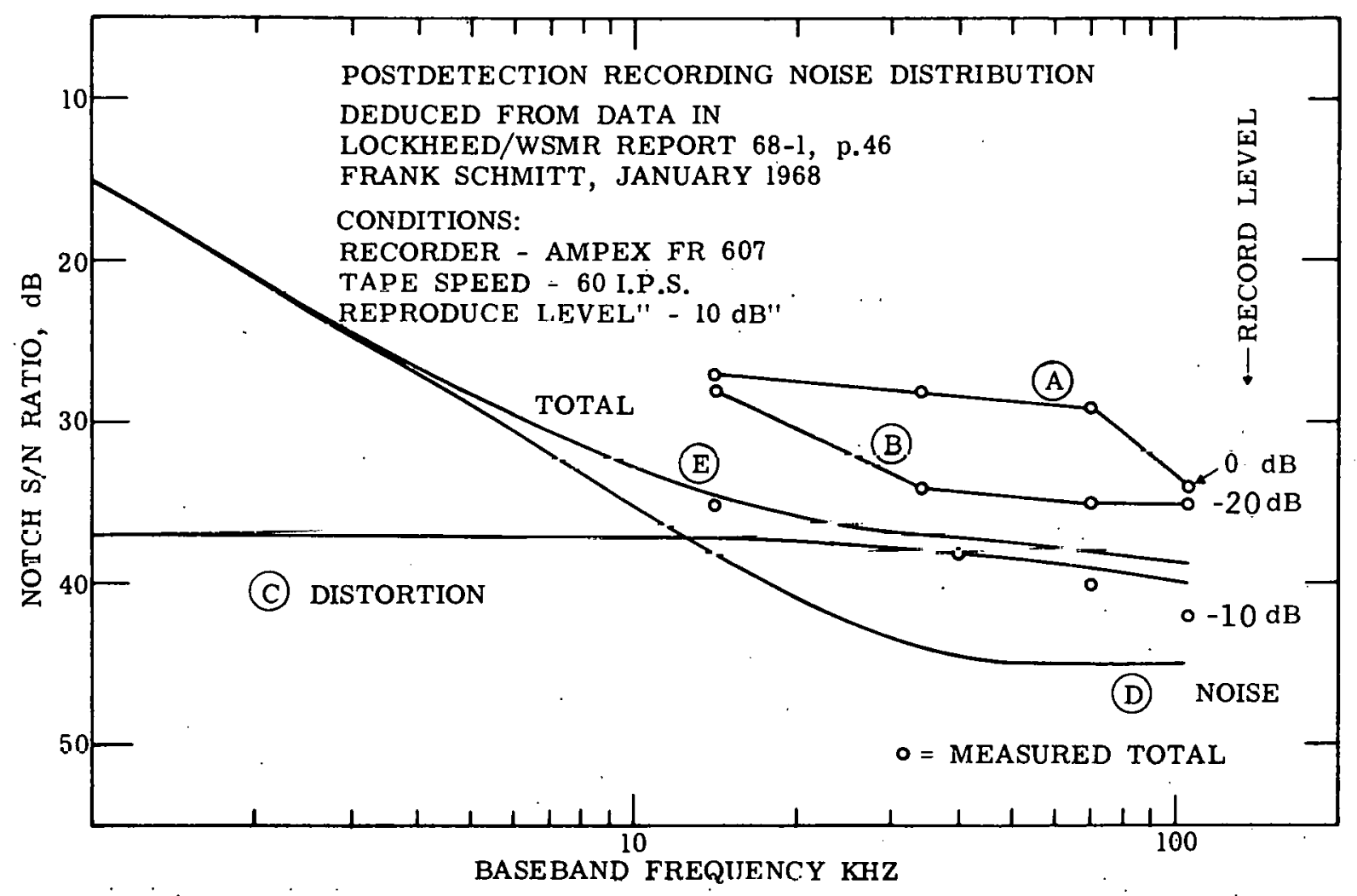

Figure 2.3-1 Post detection recording noise distribution

Figure 2.3-1 shows the variation with notch frequency at $-20 \mathrm{~dB}$, which is characteristic of tape reproducer noise spectra (the $105 \mathrm{kHz}$ notch insertion loss should not affect this measurement). Asauming this is so, the noise should vary as $-6 \mathrm{~dB} /$ octave at low frequencies because of the reproduce equalization. Lowering the noise-to-signal ratio $10 \mathrm{~dB}$ and extrapolating to low frequencies with this model gives Curve $D$ for reproduce noise at $-10 \mathrm{dR}$ record level, Combining these curves puwerwisc gives Curve $\mathrm{E}$ for the calculated total noise for this record 1 evel. Measurements at the notch frequencies for $-10 \mathrm{~dB}$ shows fair agreement with the calculations from the simple models, so the low frequency extrapolation appears val.i.d.

It is interesting to note that the noise level at the pilot frequency $1.5 \mathrm{kHz}$ is $17 \mathrm{~dB}$ higher than that measured at the $14 \mathrm{kHz}$ notch. If these lengthy calculations are to be avoided in future tests, it is advisable to have a few lower notch frequencies and lower noise analyzer frequencies. A simple extrapolation in frequency is useful but risky if the relative amount of noise and distortion is not also determined. Here we were fortunate in having a large number of data points 
with which to work. Also the second-power model is not always appropriate. For example, variation of reproduce level showed the effects of higher power terms at higher reproduce levels than the $-10 \mathrm{~dB}$ of the above data.

\section{Predetection Tape Link Noise and Distortion}

The predetection tape link noise model is plotted. in Figure 2.3-2. The circled points are derived from data on pages 38 and 53 of Reference 1 , by subtracting the noise due to the radio link from the noise measured on a system consisting of radio link with predetection recording and reproduction. The measurements were made using a carrier with no modulation. The recorder was an Ampex FR-607, operating at 120 inches per second.

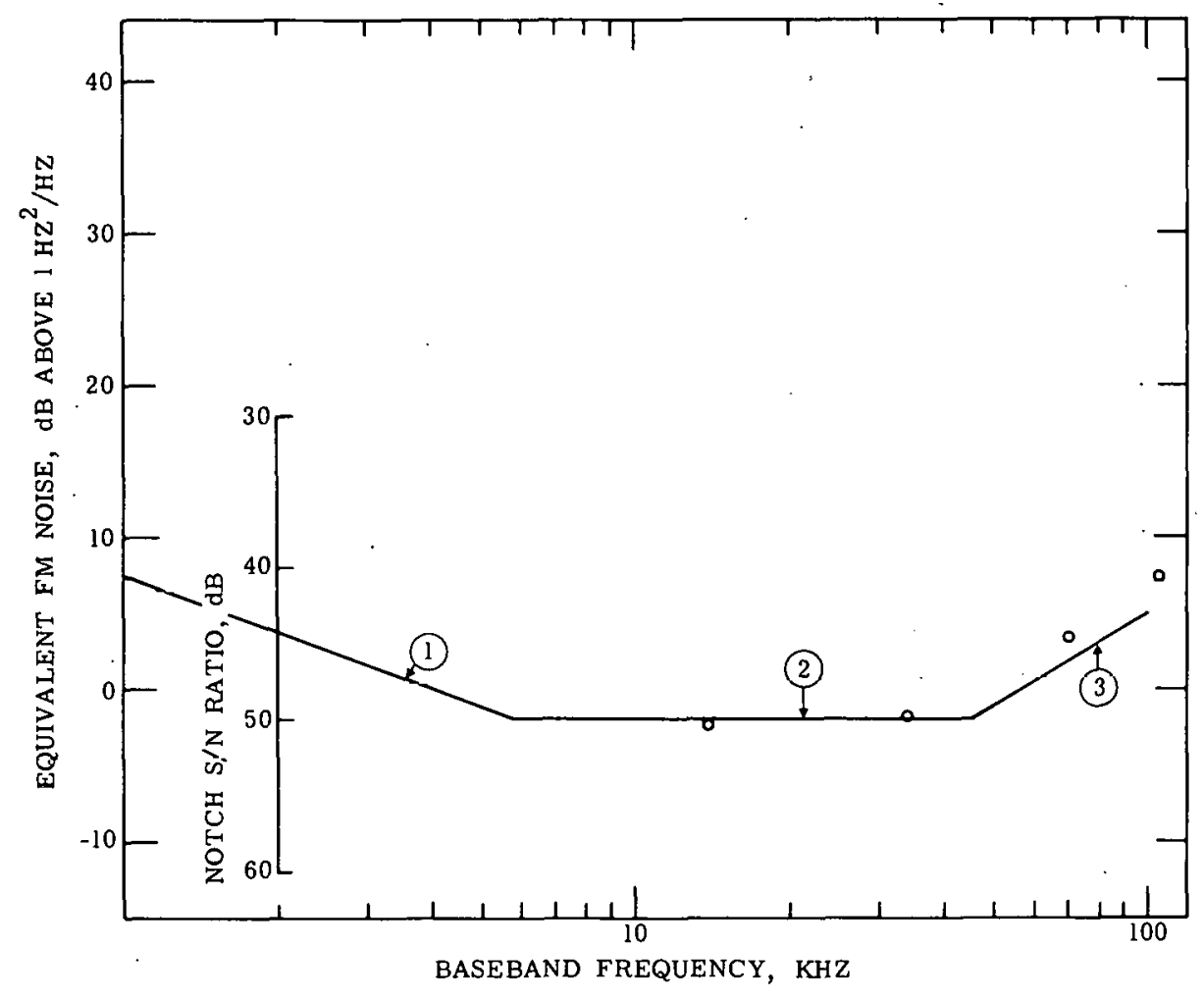

Figure 2.3-2 Predetection recording noise model

The line segment labeled (1) represents predicted baseband noise due to flutter, based on flutter model A-1 of Section 2.4, operating on a $450 \mathrm{kHz}$ carrier. Examination of the TBE spectrum plots in Section 2.4 will show that this model departs several decibels from the measured 
data at various frequencies, but the effect on overall DSB system errors will be small because the resulting noise is small compared to other system noises.

The line segment labeled (2) is fitted to the measured points in this region of the baseband. The line segment labeled (3) is calculated on the reasonable assumption of tape link predetection noise, in $1000 \mathrm{~Hz}$ bandwidth, $65 \mathrm{~dB}$ below the predetection carrier level. Actually, the noise is not flat in the band of the predetection signal, but rises toward the high end, preventing practical calculation of the FM demodulator output noise for the case of a modulated signal. This is not serious, however, because of the relatively low contribution of this noise source.

The equivalent FM noise level will probably be independent of carrier modulation over a small range of variation, but will probably change with recording signal level. No data has been found on the effect of these changes for the predetection case.

A curious effect on predetection tape link noise was reported in Reference 4. The bandpass filter at the input to the playback FM demodulator had a significant effect on the baseband notch signal-tonoise ratio; even though the noise components rejected by the filter would be expected to cause baseband noise falling well outside the range of frequencies covered by the notch measurements. This indicated the advisability of using some predetection filtering on playback, but of such a nature as to produce little additional distortion due to delay variations across the pass band. Filters designed for telemetry subcarrier discriminators (used for predetection playback in some cases) are likely to be superior in this respect to those used in telemetry receivers.

Distortion in predetection tape links is dependent on the methods of frequency conversion and playback demodulation, and is discussed in some detail in Reference 4. The best results reported to date have been gotten using a telemetry subcarrier discriminator to demodulate the tape output, rather than using up-conversion back into the radio link receiver. It also appears advisable to use the limiter in the receiver prior to recording, from the point of view of noise as measured by notch-noise techniques. When this approach is used the distortion contribution from 
a reasonable instrumentation recorder is appreciably lower than the contribution of static and filter distortion due to modern telemetry radio links. These comments are based on limited data, using baseband width under $100 \mathrm{kHz}$, rms carrier deviation under $80 \mathrm{kHz}$ and $120 \mathrm{i} . \mathrm{p} . \mathrm{s}$. tape speed.

The tests reported on page 38 and 53 of Reference 1 also give notch signal-to-noise ratios using $78 \mathrm{kHz}$ rms modulation with a random baseband extending from 12 to $108 \mathrm{kHz}$. Curve (1) of Figure 2.3-3 shows this data for the radio link. Curve (2) shows the data for the radio link combined with the predetection tape link, using direct demodulation rather than up-conversion on playback. Each of these data has been modified by subtracting the corresponding noise contribution measured without carrier modulation. The difference between these two curves might be used to calculate the distortion contribution of the predetection link, and is plotted as curve (3). Unfortunately this difference is smal1, and is negative at $108 \mathrm{kHz}$, so that curve (3) is not very dependable.

Curve (4) is obtained from the combination of down-converter and tape link distortion reported on pages 115 and 127 of Reference 4, for which the rms carrier deviation was $53.3 \mathrm{kHz}$ and the top of the random baseband at $85 \mathrm{kHz}$. The equipment was not identified. Direct demodulation was used on playback, and the tape speed was 120 i.p.s. as before. This data is more accurately due to the predetection link that is the calculation of curve (3). It is not known how to extrapolate it to the $80 \mathrm{kHz}$ modulation used for modeling in this report.

From the above data it seems reasonable for the predetection link distortion to lie below the line (5), which is $6.3 \mathrm{~Hz}^{2} / \mathrm{Hz}$ of equivalent FM modulation density. This is a small contribution to the total baseband noise at all frequencies of interest, and no effort was made to obtain a more accurate model.

When radio links are produced which have lower noise and distortion levels, these predetection levels may become of interest and can be refined by a few more measurements. 


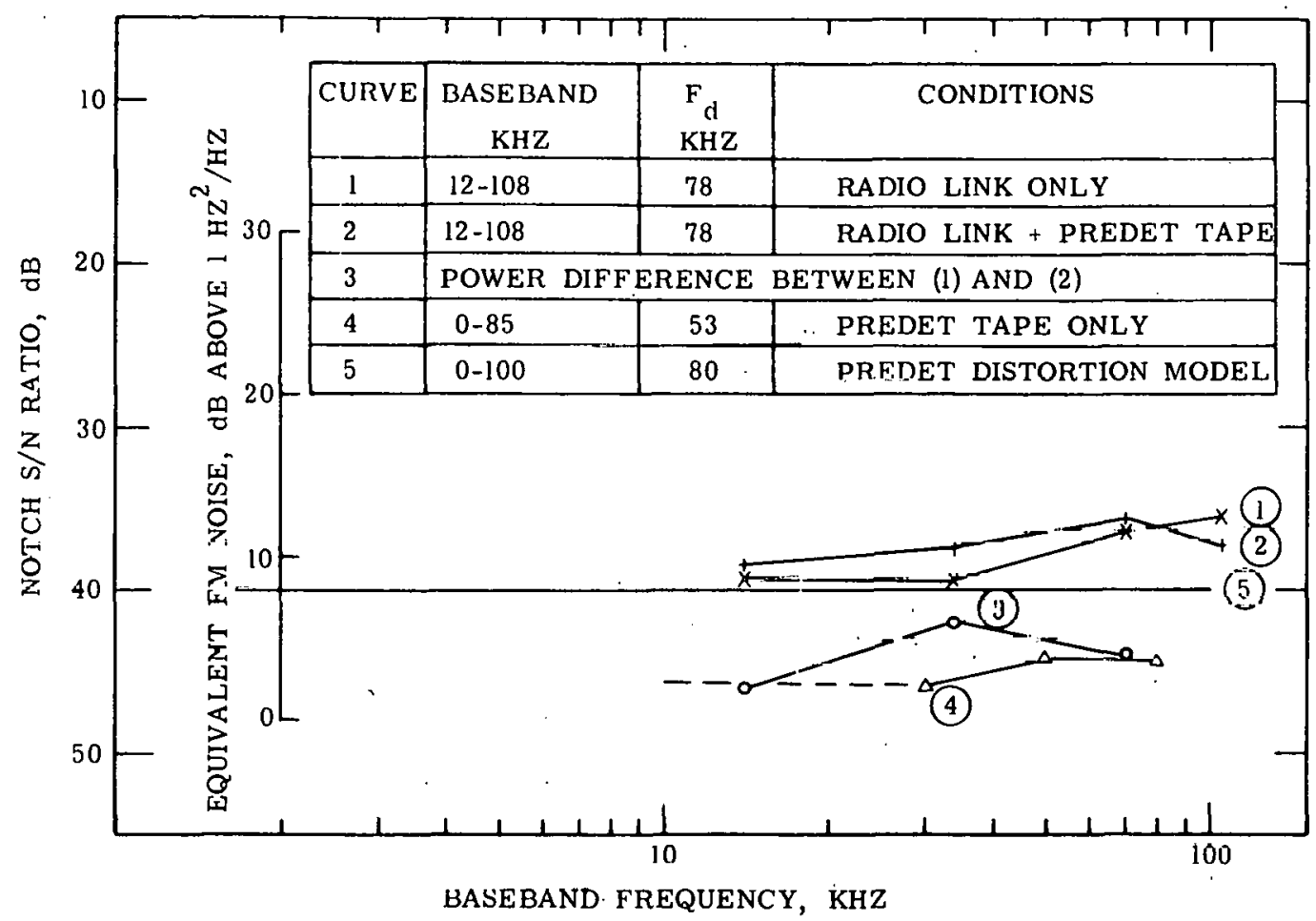

Figure 2.3-3 Predetection tape 1ink distortion tests

\subsection{Tape Link Flutter Characteristics}

Tape Speed Error Relationships

A constant-frequency sinusoidal signal of frequency $f_{0}$ is recorded on magnetic tape at a nominal tape speed $S_{O}$. On playback at a nominal tape speed $S_{0}$ the signal exhibits frequency modulation as a resilt of speed variations on record and reproduce. The combination of recording equipment, reproducing equipment, and tape will be called a "magnetic tape 1ink" or "tape 1ink."

The total effective speed variation of the tape link is deduced from a measurement of the frequency variation of the reproduced signal. The mean or steady speed tape error will be ignored in the present discussion, and that portion of the variation whose spectrum extends upward from about one.Hertz will be called "flutter." Its instantaneous value, $\mathrm{X}$, is found from the reproduced frequency relationship:

$$
f=f_{0}(1+X)
$$


The flutter variable, $x$, as defined here is in per-unit, although it is commonly expressed in percent. The spectral distribution of the variable flutter is of considerable interest. The mean square density is expressed here as the variable $x^{2}$, with the dimension (per unit) ${ }^{2} /$ Hertz.

The phase modulation of the reproduced sinusoid is the time integral of the frequency modulation, $f_{0} X$. Consider a sinusoidal flutter component:

$$
X=A \cos \omega_{\mathfrak{f}} t
$$

The peak frequency deviation is $f_{O^{A}}$. The phase variation is the integral of the frequency deviation in radian/second:

$$
\begin{aligned}
\emptyset & =\int \omega_{\mathrm{o}} A \cos \omega_{\mathrm{f}} t \mathrm{dt} \\
& =\frac{\omega_{\mathrm{o}} \mathrm{A}}{\omega_{\mathrm{f}}} \sin \omega_{\mathrm{f}} t=A \frac{f_{\mathrm{o}}}{f_{f}} \sin \omega_{\mathrm{f}} t
\end{aligned}
$$

The peak phase deviation is $A f_{0} / f_{f}$, where $f_{f}$ is the frequency of the flutter component. The time base error (TBE) can be defined in terms of the time of occurrence of zero-crossings of the modulated wave, due to the flutter. The instantaneous time variation, $Z$, is defined by:

$$
\emptyset=\omega_{\mathrm{O}} \mathrm{Z}=2 \pi \mathrm{f}_{\mathrm{O}} \mathrm{Z}
$$

For the sinusoidal case, Equations 2.4-3 and 2.4-4 give:

$$
Z=\left(A / \omega_{f}\right) \sin \omega_{f} t \text { seconds }
$$

from which the peak $\mathrm{TBE}$ is $\mathrm{A} / \omega_{\mathrm{f}}$. Hence the transfer ratio from sinusoidal per unit speed variation to TBE magnitude is $1 / \omega_{f}$. The mean square spectral density, $z^{2}$, of a random TBE can therefore be obtained from the speed variation spectral density by the relationship:

$$
z^{2}=x^{2} / \omega^{2}=x^{2} /(2 \pi f)^{2}
$$

The mean square frequency deviation spectrum is $\mathrm{f}_{0}{ }^{2}$ times the speed variation spectrum, so that the speed spectrum is of most interest in FM demodulation systems. The spectrum of the input phase variation is $\omega_{0}{ }^{2}$ times the TBE mean square spectrum. This latter spectrum is of interest in PLL tracking error calculations. 


\section{Conversion of Flutter Meter Measurements}

There are two flutter spectrum analyzers on the market (MICOM 8300-W and Ampex TU-40) whose measurement bandwidth varies with tuning, keeping a constant percentage bandwidth. These analyzers make use of the voltage output from a frequency discriminator as an indication of instantaneous tape speed variation. The discriminator input is the reproduction of a constant-frequency test signal originally recorded on the test tape. The analyzer filters this output through a handpass filter and measures the voltage level. which is exceeded by the filtered uucpur a chosen traction of the time on the average. The meter readings obtained are expressed in terms of percent speed variation, and labeled peak-to-peak with one sigma, two sigma, and three sigma, limits depending on the fraction of time chosen. The term sigma refers to standard deviation of an assumed Gaussian random flutter. In what follows the Gaussian random assumption will be used, although it is subject to some inaccuracy.

The development of flutter models for calculation of phase lock loop tracking errors requires the conversion of various spectral measurements into a common system of units. The more convenient is the mean square time base error (TBE) spectral density, expressed here as $z^{2}$ in second ${ }^{2} /$ Hertz. In terms of flutter (speed variation) spectral density. $x^{2}$, this is glven by equation $(2.4-6)$, where $x^{2}$ is in (per unit) $2 /$ Horts. The value of $x^{2}$ is found by conversion of the flutter meter readings.

A peak-to-peak meter reading, $R$, with 2-sigma limits is four times the rms value in percent ( $r m s$ may he substituted for standard deviation because the mean through the filter is zero). The per-unit variation, peak-to-peak, is $R / 100$. The per-unit rms variation is $R /(4 \times 100)$ with the 2-sigma measurement switch setting. The mean square per-unit variation divided by the analyzer bandwidth is the spectral density. For an m-sigma peak-to-peak reading, the flutter density is:

$$
\mathrm{x}^{2}=\mathrm{R}^{2} / \mathrm{B}(100 \mathrm{~m})^{2} \quad(\text { per-unit) })^{2} / \text { Hertz }
$$

where $B$ is the bandwidth in Hertz. 
Some of the measurements used in the present work were taken with a flutter meter in which the bandwidth is 0.1 times the spectral frequency, $f$. In this case the conversion to flutter density is:

$$
\mathrm{x}^{2}=\mathrm{R}^{2} / 0.1 \mathrm{f}(100 \mathrm{~m})^{2}\left(\text { per-unit) }{ }^{2} /\right. \text { Hertz }
$$

The value of the TBE spectral density for these conditions is:

$$
\mathrm{z}^{2}=\mathrm{R}^{2} / 0.1 \mathrm{f}^{3}(2 \pi)^{2}(100 \mathrm{~m})^{2} \text { second } \mathrm{s}^{2} / \text { Hert } \mathrm{z}
$$

Much of the flutter data processed in the present work was taken with the 2-sigma meter setting, so a nomograph was constructed to provide the conversions of Equations 2.4-8 and 2.4-9 with m equal to 2 . This nomograph is Figure 2.4-1.
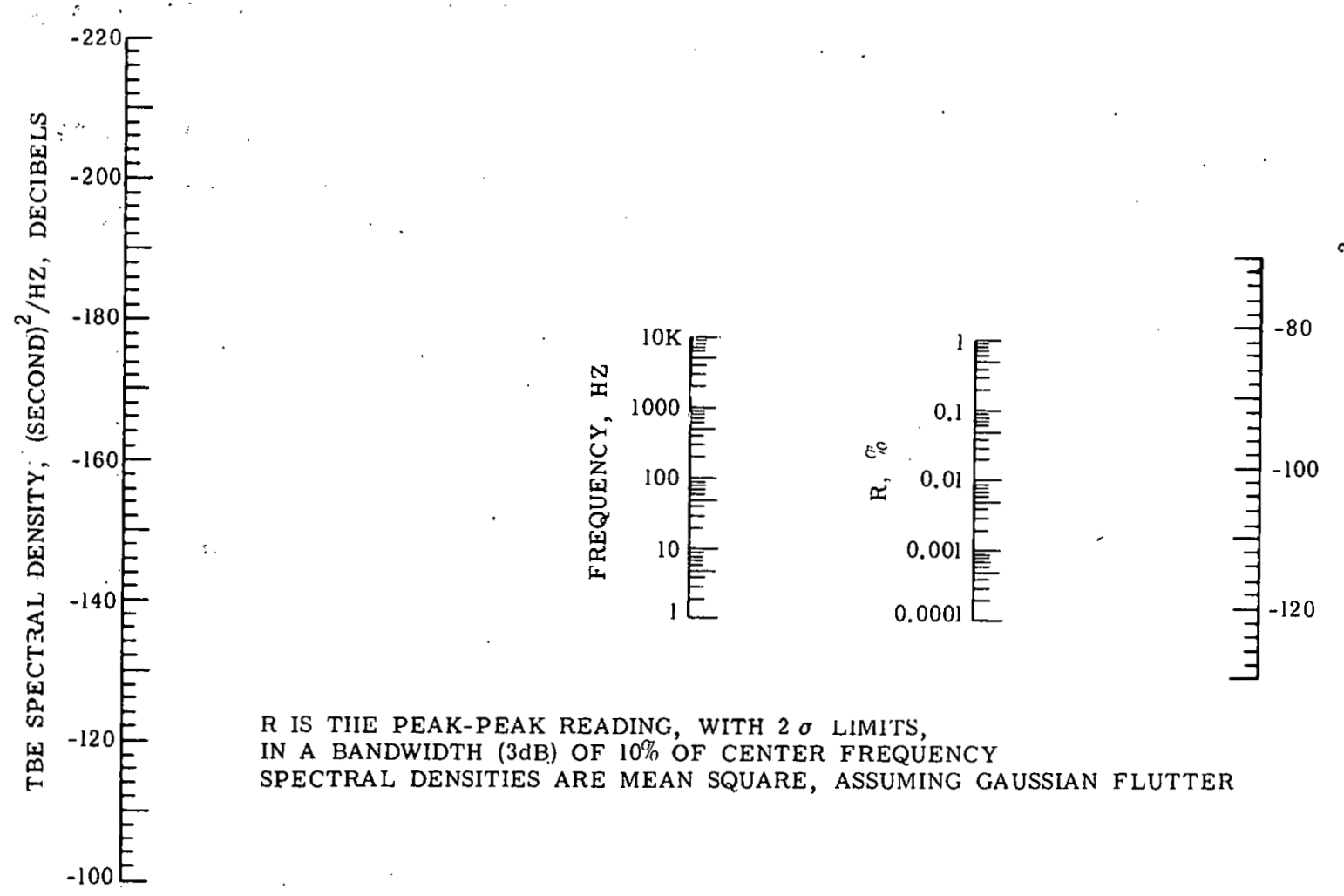

$R$ IS TIIE PEAK-PEAK READING, WITH $2 \sigma$ LIMITS,

IN A BANDWIDTH (3dB) OF 10\% OF CENTER FREQUENCY

SPECTRAL DENSITIES ARE MEAN SQUARE, ASSUMING GAUSSIAN FLUTTER

Figure 2.4-1 Flutter and TBE spectral density from Ampex TU40 readings 


\section{Tape Link Flutter Characteristics}

During the course of the work reported here, unpublished flutter measurements were received from Sandia Laboratories, Albuquerque, and used to develop the spectral models used in predicting phase lock loop tracking errors. Three different recorder models are represented, identified here as recorders A, B, and C. Brief review of unpublished data from other sources on other models indicated that these cover a reasonable range of performance. Measurements from a flutter spectrum analyzer were processed as explained in this section and are plotted in Figures 2.4-2, 2.4-3, and 2.4-4. The tape speed on record and reproduce in a11 cases was 60 inches/second. Three tracks were measured on each machine. Recorders $A$ and $B$ used one-inch tape, and were operated without tape-track servo control on playback. Recorder C used 1/2-inch tape, with servo control operating from track 3 .

On each of the TBE spectrum plots a power-law line has been drawn which lies above most of the points between about 40 and $400 \mathrm{~Hz}$. For the phase' lock loop parameters likely to be encountered in the current

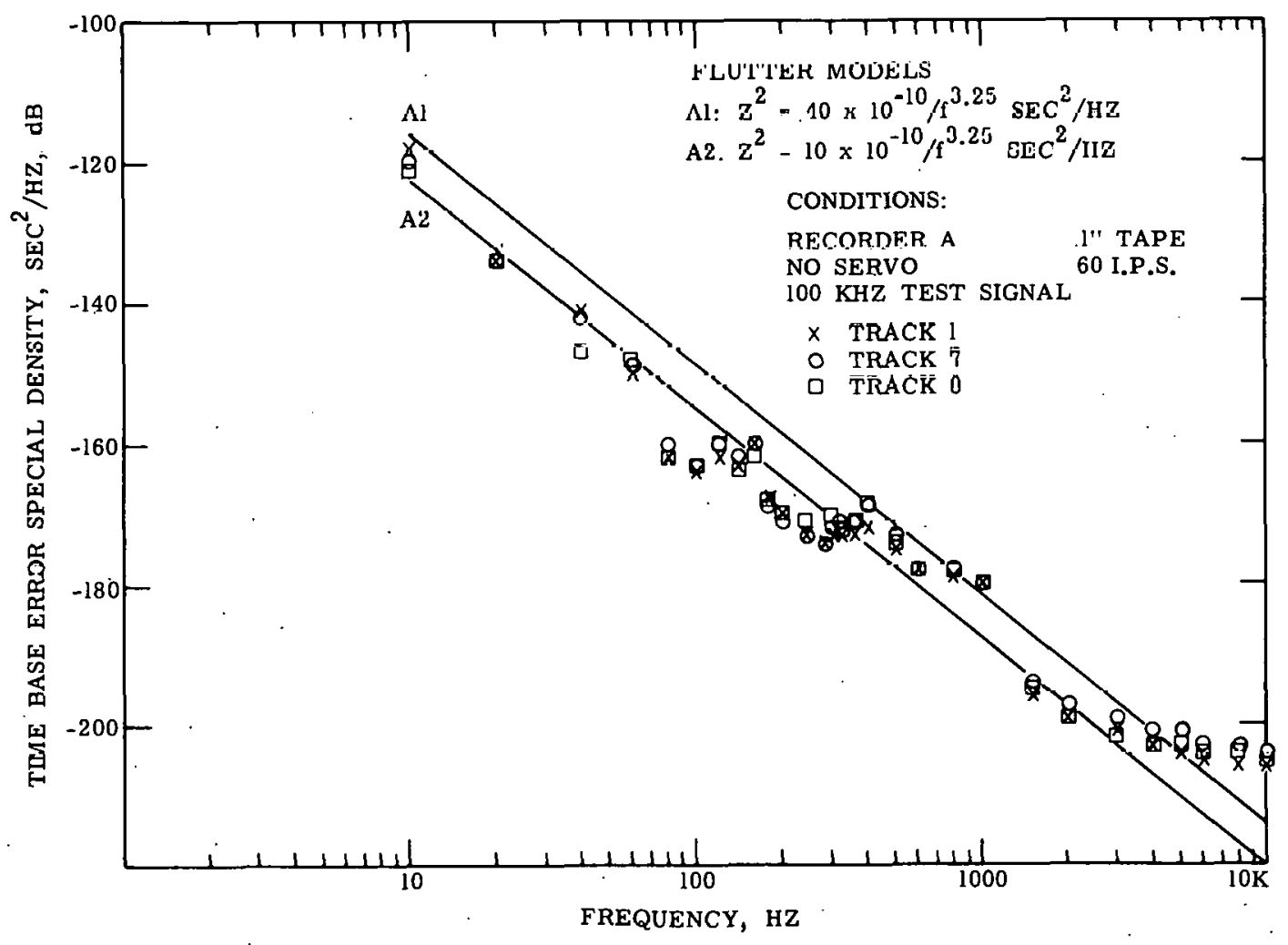

Figure 2.4-2 Tape link flutter data 


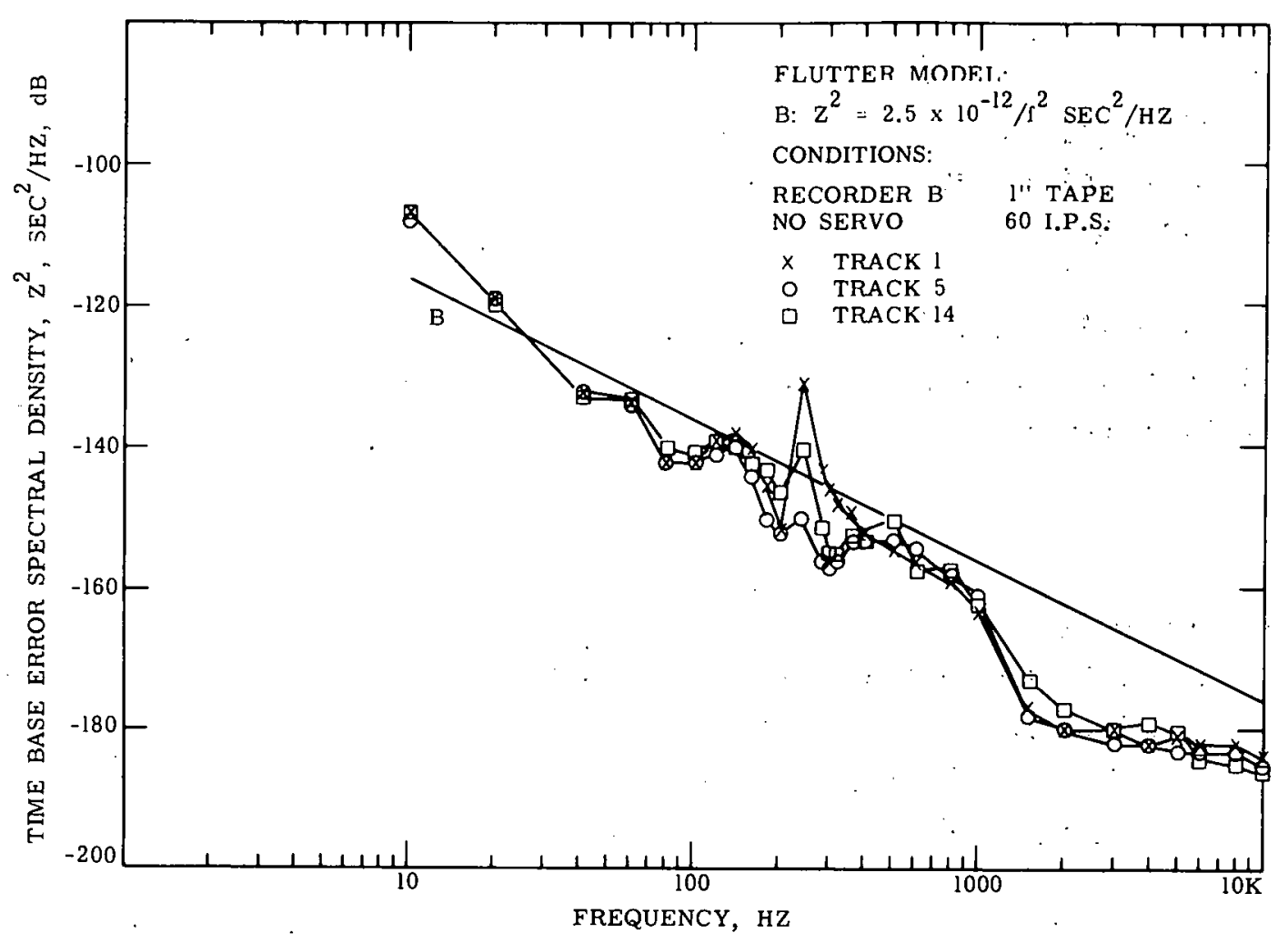

Figure 2.4-3 Tape 1ink flutter data

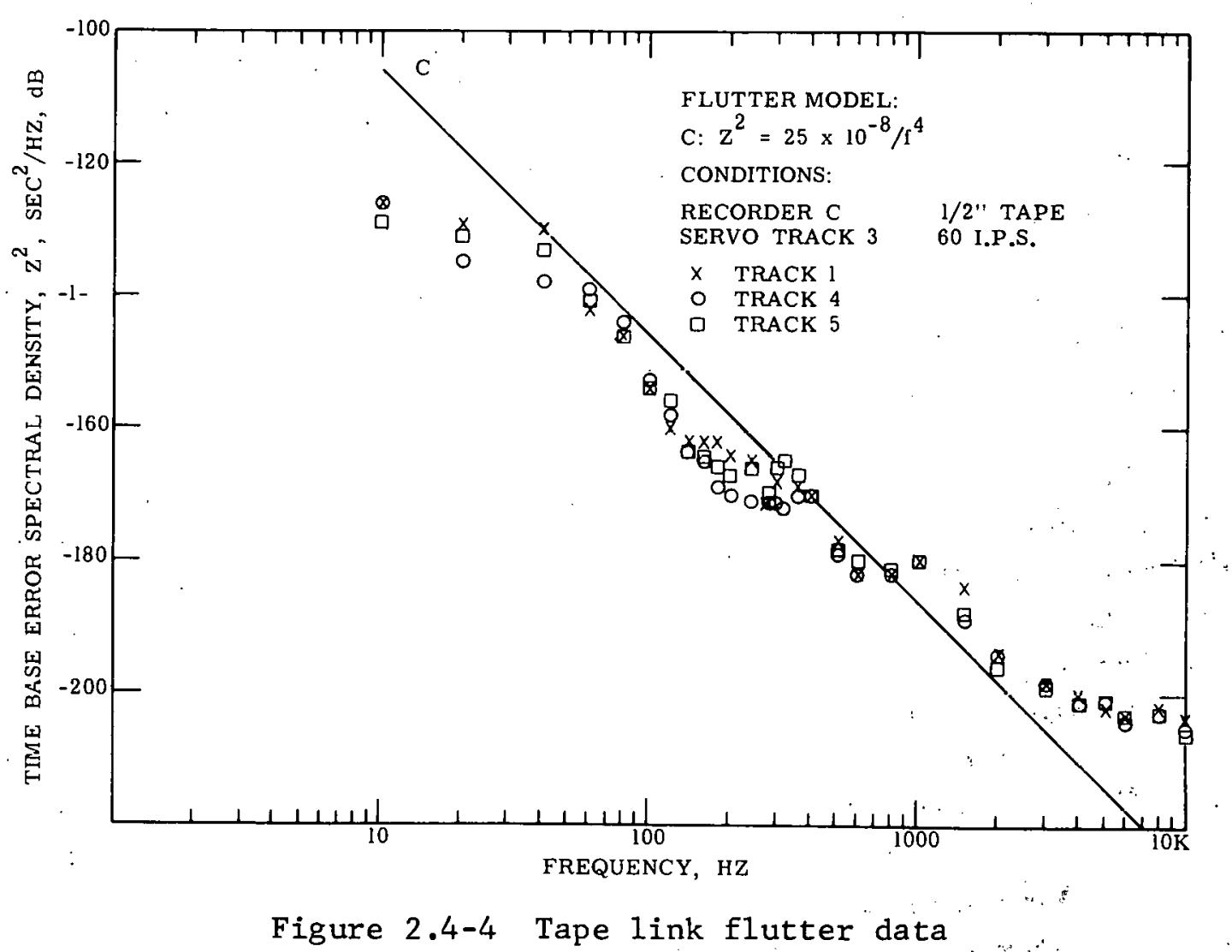


DSB telemetry work, the spectrum below $40 \mathrm{~Hz}$ will be tracked by the loop, and the spectrum above $400 \mathrm{~Hz}$ will not cause appreciable error in the predictions.

The line labeled A-2 is taken from Figure 1 of Reference 2. It makes a good envelope for the data on Recorder A out to $300 \mathrm{~Hz}$, and will be used further as Flutter Model A-2. Its equation is:

$$
z^{2}=10^{-9} f^{-3.25} \text { second }{ }^{2} / \text { Hertz }
$$

The line labeled A-1 is simply A-2 moved up to cover the points at 400 $\mathrm{Hz}$ and above, to indlcate that $\mathrm{A}-2$ is within $6 \mathrm{~dB}$. of the higher points up to $1 \mathrm{kHz}$. The contribution of these points will be negligible, however, and they will be ignored. It is interesting to note the agreement among the spectra from three separate tracks on the plot for Recorder A.

On recorder $B$ again the tracks exhibit close agreement except in the neighborhood of $240 \mathrm{~Hz}$, where there is a spread of $19 \mathrm{~dB}$. The two tracks which were worst in this region were ignored in choosing Flutter Mode1 B, which is plotted from:

$$
\mathrm{z}^{2}=2.5 \times 10^{-12} \mathrm{f}^{-2} \text { second }{ }^{2} / \text { Hertz }(2.4-11)
$$

The data on Recorder C lies below a line for Model C given by:

$$
\mathrm{z}^{2}=25 \times 10^{-8} \mathrm{f}^{-4} \quad \text { second }^{2} / \text { Hertz } \quad(2.4-12)
$$

It is interesting to note that track 4 is the best of the three, although it is on the opposite head stack from the servo control signa1. which was on track 3 .

Thiese flutter models were subject to criticism, of course, because they lie above most of the effective data points. A line 6dR lower would lie below most of the points. Offsetting this apparent conscrvatiam is the Gaussian random assumption, which is used in obtaining spectral density from a point measurement. If a measured component were sinusoidal, its rms value would be closer to the 2-sigma reading than has been assumed. The random spectrum inferred from an unsuspected sinusoid depends upon the analyzer bandwidth, while the indicated level does not.

An example of the inaccuracy due to sinusoidal components can be gotten by examining the measurement for track 14, Recorder B, at $240 \mathrm{~Hz}$, 
which gave a peak-peak reading of $0.03 \%$. If this is due to sinusoidal flutter, it will phase-modulate a $100 \mathrm{kHz}$ carrier 0.044 radians rms. A phase lock loop with natural frequency in the neighborhood of $240 \mathrm{~Hz}$ might exhibit 0.7 times this as phase error. The same PLL will exhibit a phase error of only 0.040 radians rms due to the whole of Flutter Model B.

Predictions based on these flutter models may be risky, but they are all we have with the presently available information. In the future it is advisable to obtain cumulative phase error data, using particular phase lock loops to measure untracked TBE.

A11 of the flutter models are collected in one plot in Figure $2.4-5$.

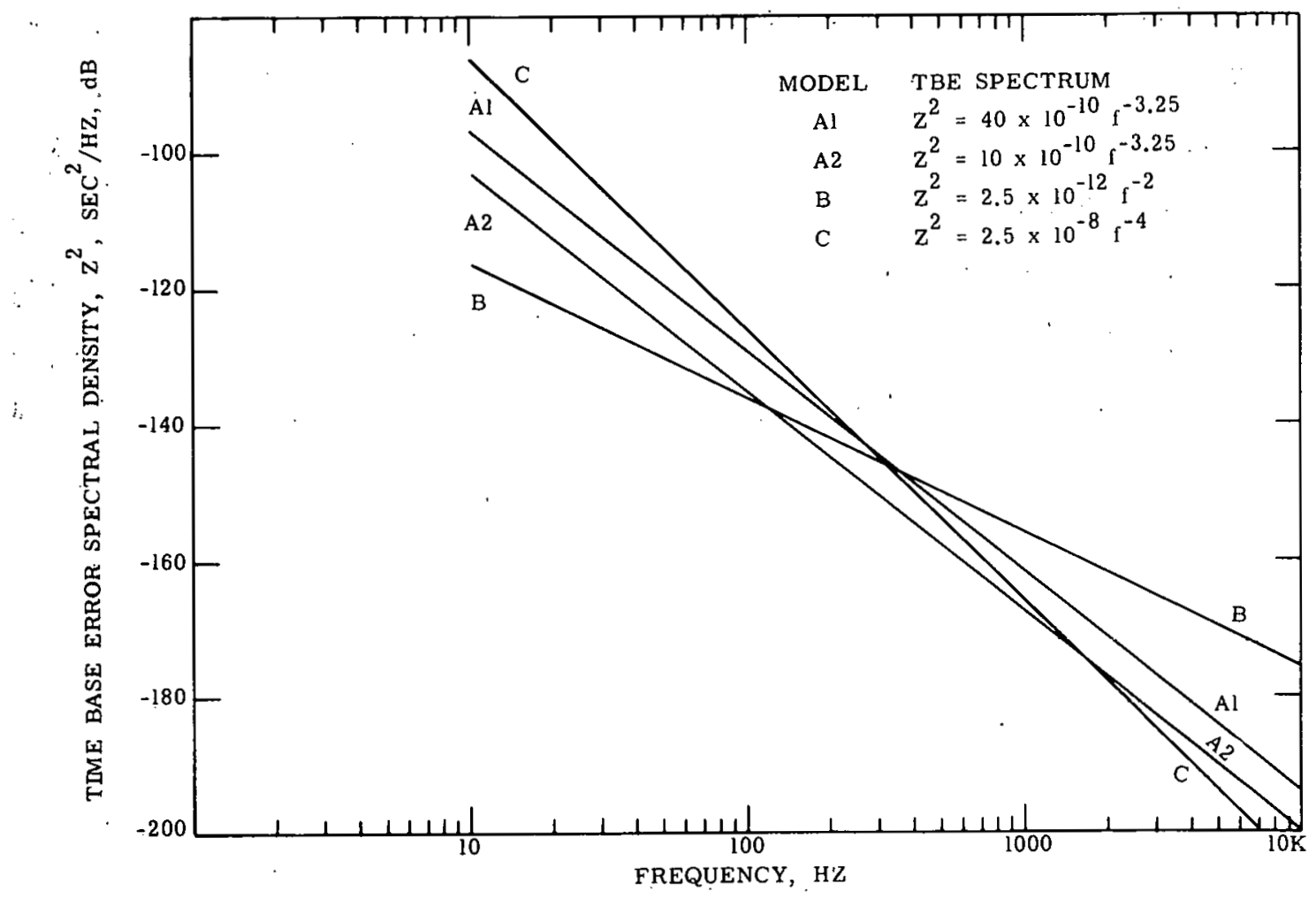

Figure 2.4-5 Tape link flutter models 


\section{Symbols Used in Section 2.4}

A Amplitude of a sinusoidal tape speed variation, per unit

Bandwidth (resolution) of. flutter spectrum analyzer,

Frequency of a recorded sinusoida1, Hertz

$2.4-1$

f Frequency (in general), Hertz

Frequency of a reproduced sinusoid, Hertz

$2.4-1$

$\dot{t}_{\mathrm{f}}$

Frequency of a sinusoidal tape speed variation, Hertz

$2.4-3$

m Nomber of standard deviatiuns, relaring to peak limits on flutter meter settings

$2.4-7$

$\mathbf{R}$

Flutter meter reading, percent peak-to-peak

$2.4-7$

$\mathrm{X}$

Instantaneous tape speed error, per unit

$2.4-1$

$x^{2} \quad$ Power spectrum of $X(t)$, (per unit) ${ }^{2} /$ Hertz

$2.4-1$

$\mathrm{Z} \quad$ Tape link instantaneous time base error, seconds

$2.4-4$

$z^{2}$

Power spectrum of $z(t)$, second $2 /$ Hertz.

$2.4-6$

$\omega_{0}$

$2 \pi \mathrm{f}_{\mathrm{o}}$, radian/second

2.4-3

$\omega_{\mathrm{f}}$

$2 \pi f_{f}$, radian/second

$2.4-3$

$\emptyset$

Pháse error of a reproduced sinusoid, radians

2.4-3 


\subsection{Radio Link Transfer Characteristics}

The baseband phase shift for various telemetry transmitter-receiver combinations has been measured at NASA/MSFC by W. H. Tranter and G. D. Weathers of the Sperry Rand Corporation (Reference 9) and at Sandia Laboratories by C. S. Johnson and R. M. Caster (Reference 8). These measurements were initiated as a result of this author's earlier comments on the significance of fixed phase errors in harmonic subcarrier DSB demodulation. These measurements have shown that RF 1ink phase errors can be reasonably small for basebands below $100 \mathrm{kHz}$, using 500 $\mathrm{kHz}$ IF bandwidth and essentially no video filtering. When the receiver video bandwidth is below, say, $250 \mathrm{kHz}$, appreciable phase error results and should be compensated by equalizing filters, or by phase adjustments in the demodulators if the operating practices permit this. It appears better to correct each receiver individually, to permit receiver switching into each demodulation station, particularly when data processing is to be done at a tape playback station.

Figure 2.5-1 shows the phase error due to the radio link receiver for the harmonic subcarrier method in which the demodulation phase reference is derived from a phase synchronization pilot signal at $64 \mathrm{kHz}$. The error is the difference between the measured phase shift at each baseband frequency and the calculated phase shift for an exactly linear phase shift line passing through the measured value at $64 \mathrm{kHz}$. Curves 1, 2, and 3 are taken from such calculations reported in Reference 9. Curves 4 and 5 are calculated from unpublished data furnished by C. S. Johnson of Sandia Laboratories, Albuquerque.

The phase error of curve 3 , it compared to curve 1 , can be attribured mainly to the 500 IF filter. Curve 4 was taken with a different transmitter than curve 1 . It has been compared with data taken with a repeller-modulated Klystron signal generator and found to be essentially identica1. Therefore the error of curve 4 does not appear to be due to the transmitter and is attributed mainly to the $250 \mathrm{kHz}$ video filter in the receiver. Adding the errors due to the $500 \mathrm{kHz}$ IF and the $250 \mathrm{kHz}$ video gives circled points, which agree moderately well with the measured data of curve 5 for these same conditions.

It may be advisable to use some video filtering to reduce the noise above the baseband which will load various baseband circuits in the system. Therefore the phase error due to the $400 \mathrm{kHz}$ video filter will be 


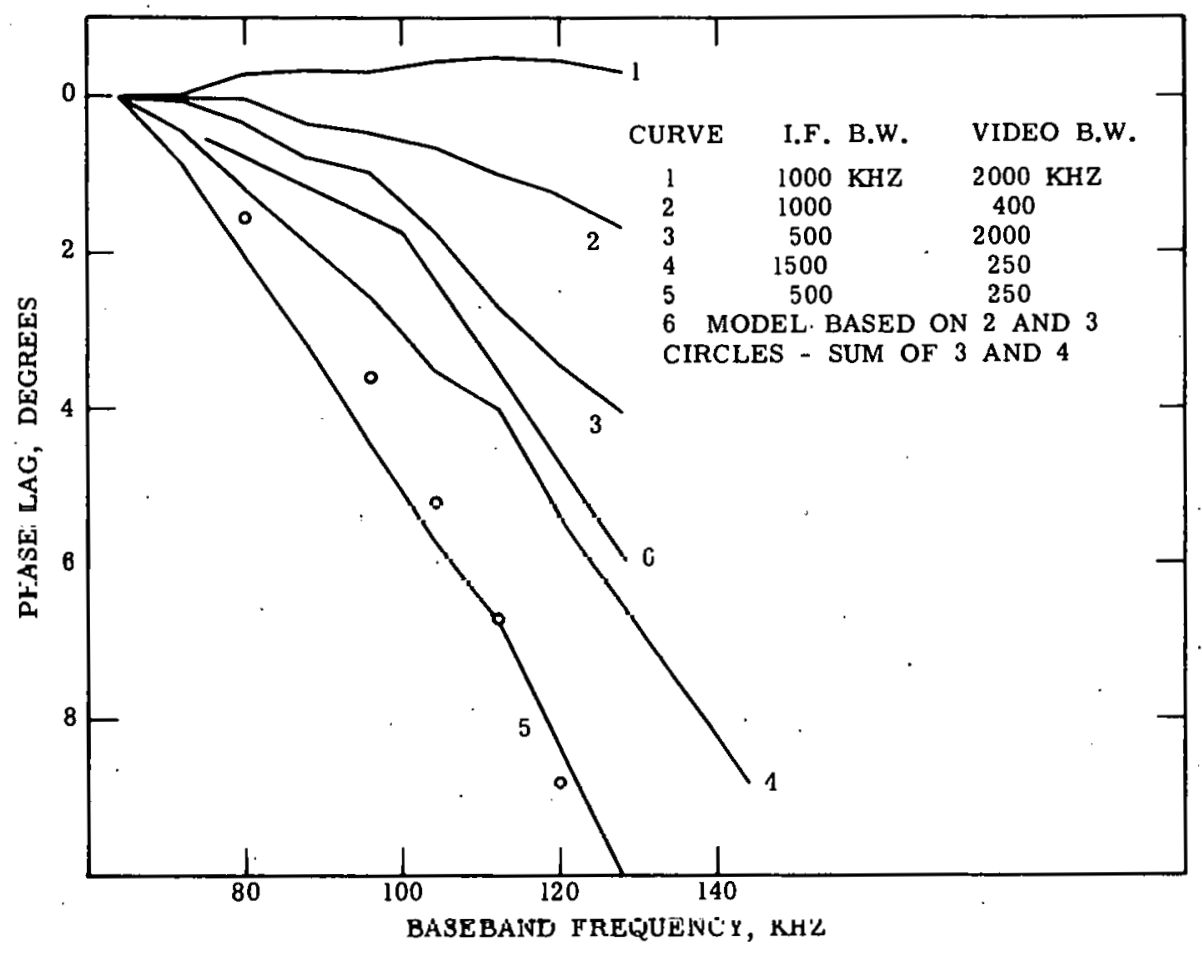

Figure 2.5-1 Radio link baseband phase error

added to $b 00 \mathrm{kHz}$ IF error to obtain the error model to he user here. Error due to the transmitter will be ignored, based on the two transmitters represented in the data of Figure 2.4-1, with the caution that other transmitters can certainly create additional errors and should be tested or specified to avoid trouble. The model for the radio link phase error is curve 6 .

Low frequency phase shift should be mentioned. Calculations using common values of low frequency response for trmp FM transmitters and telemetry receivers show no appreciable phase shift at any frequencies of interest 1 . the low baseband.

Voltage gain versus frequency data was also taken on the transmitter/receiver combinations whose phase error is plotted as curves 4 and 5 in Figure 2.5-1, and are shown in Figure 2.5-2. Curve 1 is the frequency response of the link with a $500 \mathrm{kHz}$ IF filter, curve 2 for a $1500 \mathrm{kHz}$ filter. The video filter bandwidth was $250 \mathrm{kHz}$ for both curves. The gain scale on this figure, and on Figure 2.5-3, causes the limited resolution of the measurements (around 0.5 to $1.0 \%$ ) to show up as sudden jumps which are probably not characteristic of the links. Looking at 
the gross features, however, it appears that the $500 \mathrm{kHz}$ IF-filter causes a drop of about $6 \%$ in baseband gain at $100 \mathrm{kHz}$, and a lesser but significant difference in gain at other parts of the baseband. In addition, there is a noticeable drop between the low frequency value ( $4 \mathrm{kHz})$ and the portion around $20 \mathrm{kHz}$ with both filters in Figure 2.5-2. With

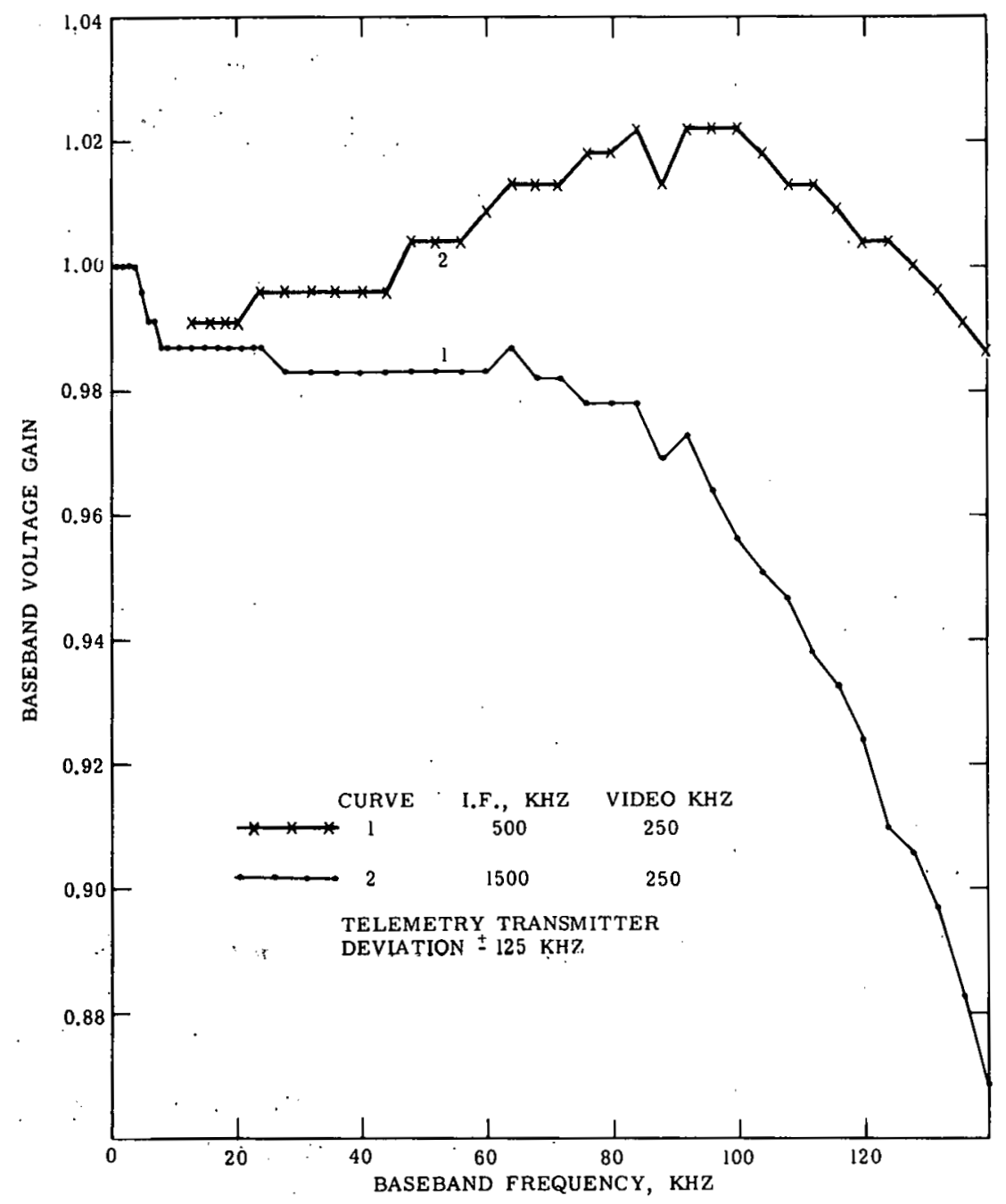

Figure 2.5-2

Radio link baseband gain variation

the wide filter the gain then rises to about $2 \%$ above the low frequency value at $100 \mathrm{kHz}$. To get some idea of the contribution of the transmitter to gain variation, the data of Figure 2.5-3 is plotted for the same receiver operating from a signal generator having a repellormodulated klystron. In this case the gain rises rather rapidly about $5 \%$ from $4 \mathrm{kHz}$ to $20 \mathrm{kHz}$, varying about 3\% peak-to-peak from 20 to 100 $\mathrm{kHz}$, with the $1500 \mathrm{kHz}$ IF filter. Inserting the $500 \mathrm{kHz}$ IF filter makes about the same amount of difference as in Figure 2.5-2, in this case 


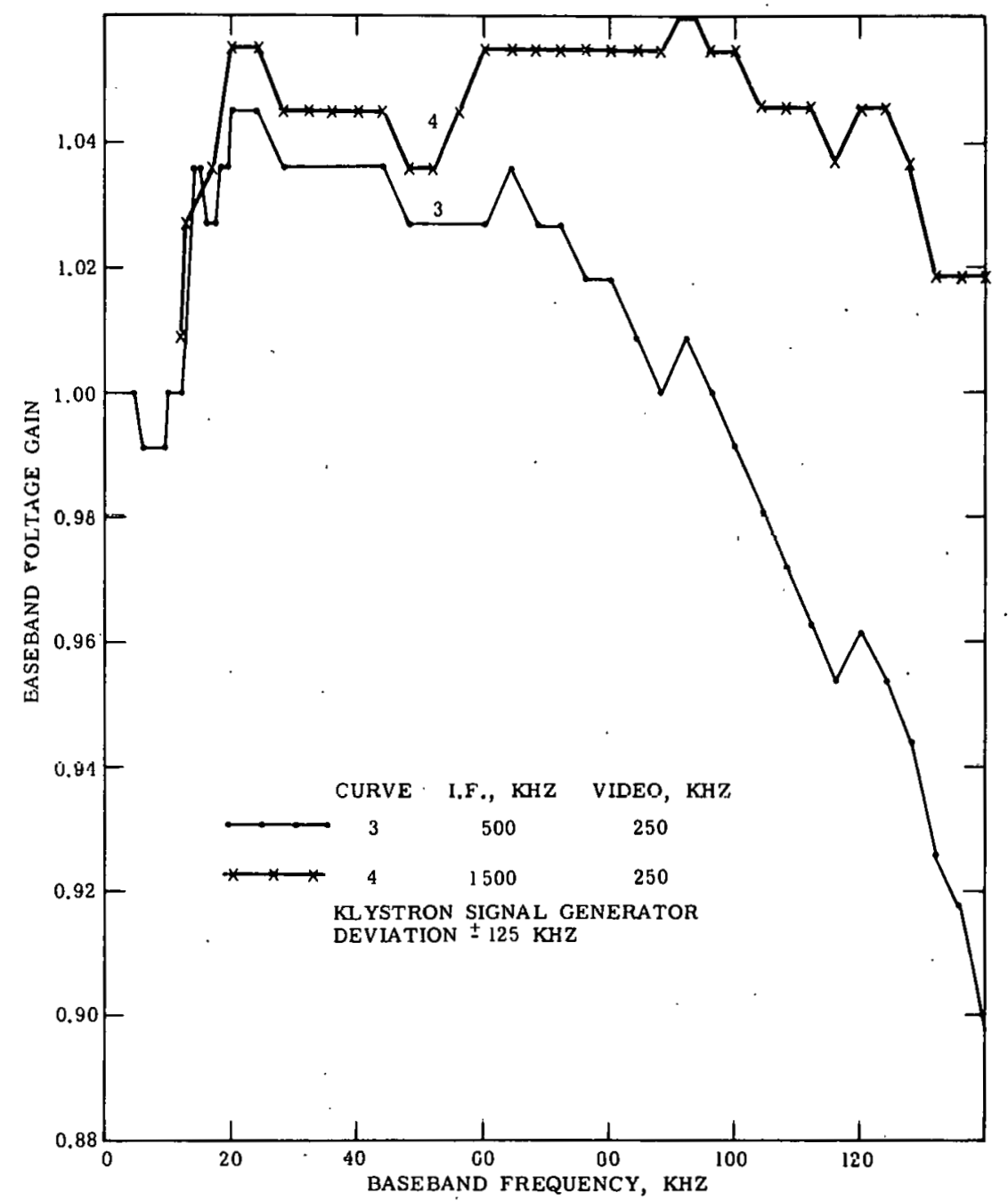

Figure 2.5-3

Radio link baseband gain variation

making the value at $96 \mathrm{kHz}$ the same as the value at $4 \mathrm{kHz}$; with a rise of about $4 \%$ in between these frequencies.

The gain variations will cause similar variations among the gains of the various subcarrier channels if not compensated with equalization or adjustment, or automatically. corrected with channel level control. If operating without channel level control, it is advisable to equalize the transmitters and receivers individually to assure interchangeability among various units. To accomplish this conveniently requires special test equipment, unless errors of several percent can be tolerated. 


\subsection{Tape Link Transfer Characteristics}

\section{Postdetection Tape Link Characteristics}

Postdetection tape links running at 60 inches per second show variations of several $\mathrm{dB}$ in amplitude transfer ratio across the baseband from $4 \mathrm{kHz}$ to $100 \mathrm{kHz}$. The amount of variation depends upon the mode of operation and the procedure used in equalization. A particular record/reproduce equipment combination may be equalized, but subsequent interchange of equipment apparently causes significant changes. Operation modes are as follows:

1. Record and playback on same machine

2. Record and playback on same model, different serial number

3. Record and playback at different speeds

4. Record and playback on different models

5. Equalize with one tape type, operate with a different tape.

Figure 2.6-1 illustrates measurements made at 60 inches per second, under various conditions. Curves 1 and 2 are for record/reproduce on the same machine in each case. The machines are from two different manufacturers. Curves 3, 4, 5, and 6 are for record and reproduce on different models in each case. Curves 3 and 4 are for different tracks, using the same equipment combination. Curves 5 and 6 are for different tracks, again using the same equipment combination. All of the curves have been normalized to the gain at $4 \mathrm{kHz}$, contemplating the use of a baseband level control pilot at that frequency without use of channel level control. The gain across the baseband exceeds $6 \mathrm{~dB}$ for some combinations, so that for most applications some sort of calibrations would be necessary in order to operate without channel level control.

The data for curves $3,4,5$, and 6 were taken from a paper by G. H. Schulze, Pan American World Airways/AFETR (Reference 10)', which discusses in some detail the difficulties in obtaining flat amplitude response with magnetic tape links under range operating conditions.

There is little data on the phase shift across the baseband of postdetection magnetic tape links. Figure 2.6-2 shows the measured lowfrequency phase characteristic of two different tape machines running at 60 inches per second. In each case record and reproduce used the same machine. The data are from E. B. Campbell and W. R. Herbert of 


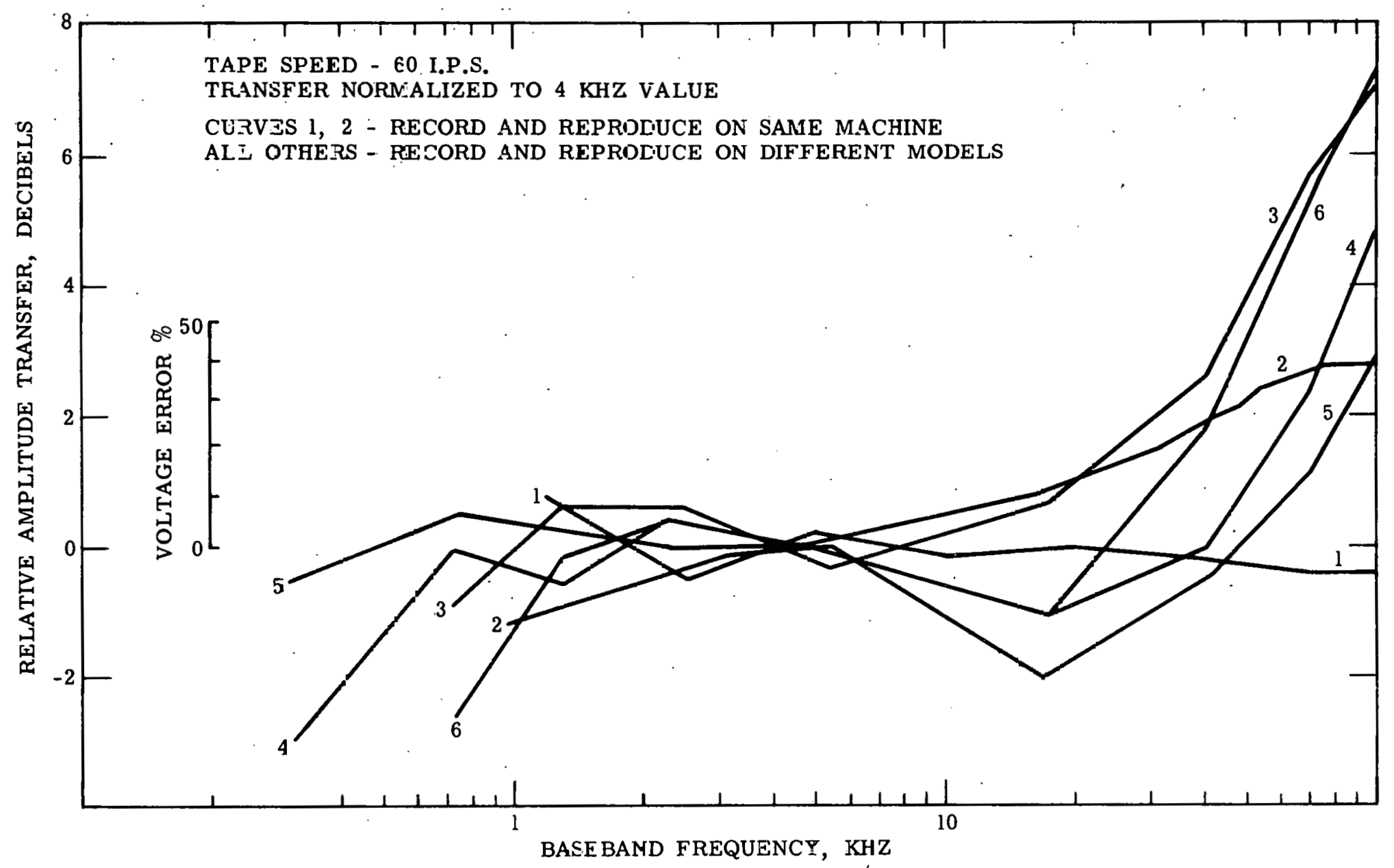

Figure 2.6-1 Amplitude trans Fer ratio, postdetection tape links 


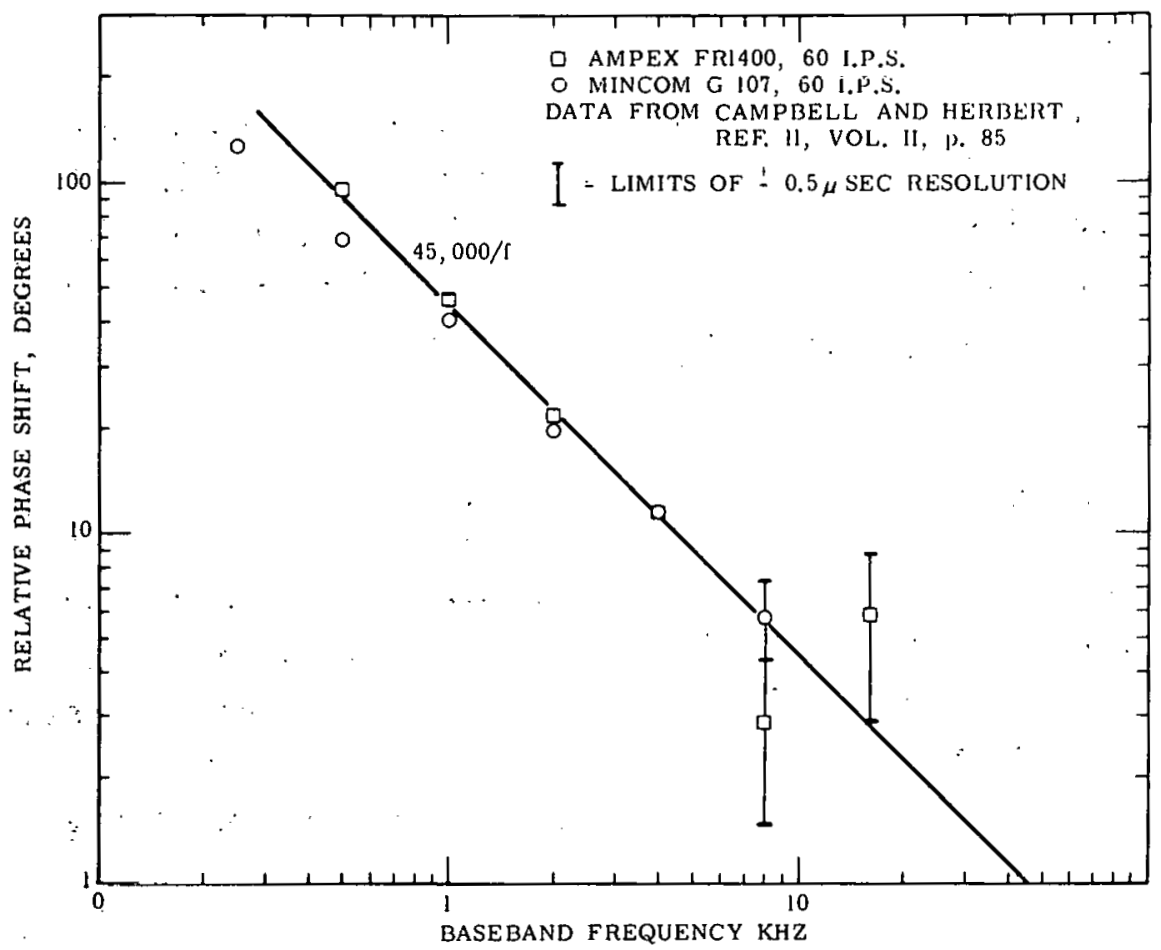

Figure 2.6-2 Baseband phase characteristic, postdetection tape links

Electro-Mechanical Research, Inc., Reference 11. The reference a1so gives amplitude variation across the baseband for the two machines. The scatter of the two points at 8 and $16 \mathrm{kHz}$ in Figure $2.6-2$ is thought to be due to the limited resolution ( 1 microsecond) of the variable delay line used in the measurements.

$\because$ The phase shift at $4 \mathrm{kHz}$ is about 11.5 degrees, which we shall see is not tolerable when the $4 \mathrm{kHz}$ pilot is used for phase reset in conjunction with a phase synchronization pilot at $64 \mathrm{kHz}$ in harmonic subcarrier operation. Equalization of this phase shift can be accomplished, but the variation due record and playback on different machines is not known.

The phase shift at $1.5 \mathrm{kHz}$ is around 30 degrees, which enough to prevent the operation of the phase reset function as normally used with independent subcarriers unless compensated in some way." In this case it appears possible to modify the phase reset function to tolerate the amount of phase shift. Reference 12 indicates the need for adjusting the independent subcarrier demodulation station for postdetection tape links with use of a test tape made on the particular machines. At first sight it would appear the various functions associated with independent subcarriers would accommodate the variations due to the tape link. 
Discussions with one of the authors of that paper indicated that the phase reset function was effected by the postdetection tape link. The baseband and channel level controls should standardize the amplitude of the pilot signals without regard to tape link transfer, so the only obvious remaining cause is the tape link phase shift.

\section{Predetection Tape Link Transfer}

The predetection tape link transfer will contain a phase and amplitude factor due to the receiver IF filter, as well as to the transmitter. These are already included in the radio link transfer discussed in Section 2.5, so the present section will be concerned only with additional components due to recording, reproduction, carrier filtering, demodulation and baseband lowpass filtering.

If the recording is done at $120 \mathrm{i} . \mathrm{p} . \mathrm{s}$. with a $450 \mathrm{kHz}$ center frequency, it appears that the record/reproduce functions will have negligible effect on the amplitude and phase transfer as measured at baseband. This includes the characteristics of the down-conversion from the receiver IF to the recording band.

On playback, it is assumed that a direct demodulator will be used, rather than up-conversion to the receiver demodulator. As discussed In Section 2.3, it is advisable to use a bandpass filter at the input to the demodulator. The bandpass filter affects the baseband phase characteristic, as was shown empirically in Section 2.5. If we consider low-index frequency modulation (first-order sidehands only) with a sinusoidal signal, we find that the odd-function departures from linearity of the filter phase characteristic cause equal phase departures from linearity in the demodulated signal. Even-function phase linearity is less prevalent than odd in symmetrical filter designs, and in addition causes negligible baseband phase error for small IF phase errors. Evenfunction IF phase departures do cause PM to AM conversion.

The important odd-function IF phase errors are also responsible for most of the baseband distortion due to the IF filters. In Sections 2.2 and 2.3 we find that the predetection tape link distortion lies well below the radio link distortion due to the IF filter. We conclude from this that the phase shift at baseband due to the predetection playback bandpass filter can be kept appreciably less than that due to the 
receiver and it will be ignored. This depends, of course, on the choice of the filter, which may be made wider than the receiver IF filter. In addition, bandpass filters used on telemetry subcarrier discriminators are generally of linear-phase designs.

The demodulator need not introduce appreciable departures from linear-phase transfer, assuming that a pulse-averaging demodulator is used. The demodulator output lowpass filter is a source of phase shift which may be significant. Video filtering is more likely to be needed on the predetection demodulator output than on the receiver output in order to remove the carrier (or twice carrier) component caused by the pulse generator in the demodulator, and to remove out-of-band noise due to the use of a wide bandpass filter ahead of the demodulator. The characteristics of the needed filter have not been determined at this time.

The amplitude transfer due to the various predetection tape link components has not been determined, although such a test can be readily carried out. There may be some variation due to the lowpass filter, and due to the equivalent filtering effect of the pulse shape used in the playback demodulator. If operation without channel level control is attempted, the variation due to these components may need calibration or equalization, taking into account the phase variation introduced by such equalization. This problem has not been covered in the present work. 


\subsection{ERROR TRANSFER RELATIONSHIPS}

\subsection{Demodulator Transfer}

In this section the term demodulator refers to a combination of multiplier and lowpass filter, not including the various level controls and reference signal sources. There are two inputs, one consisting of signal and noise, the other consisting of the demodulation reference. The signal, $e_{2}$, is represented by:

$$
e_{2}=E_{2} \sin \left(\omega_{2} t+\theta_{2}\right)
$$

where the subcarrier amplitude, $E_{2}$, may be either positive or negative, depending on the polarity of the data input signal to the channel modulator. The reference signal, $e_{5}$, is represented by:

$$
e_{5}=E_{5} \sin \left(\omega_{5} t+\theta_{5}\right) \text {. }
$$

Generally there will be a phase error between the subcarrier and reference inputs, equal to $\theta_{2}-\theta_{5}$, but under normal conditions their frequencies will be the same. For a steady subcarrier signal, the resulting dc signal output, $e_{6}$, will be:

$$
\mathrm{e}_{6}=\frac{\mathrm{E}_{2}}{\sqrt{2}} \cos \left(\theta_{2}-\theta_{5}\right)=\sqrt{\mathrm{P}_{2}} \cos \left(\theta_{2}-\theta_{5}\right)
$$

where the $\sqrt{2}$ is inserted for convenience to make the mean square signal output equal to the mean square signal input, and $\mathrm{P}_{2}$ is the mean square subcarrier voltage corresponding to $e_{6}$.

We are also concerned with the transfer of input random noise to the output. A sinusoidal noise component will cause an output, $e_{n}$ given by:

$$
e_{n}=\frac{E_{n}}{\sqrt{2}} \cos \left[\left(\omega_{n}-\omega_{j}\right) t+\left(\dot{\theta}_{n}-\theta_{5}\right)\right]
$$

where $E_{n}$ is the amplitude of the sinusoidal noise component at frequency $\omega_{n}$. The peak output is $E_{n} / \sqrt{2}$, indicating that the mean square transfer ratio is one-half for signals not coherent with the local reference. When there is a noise power density, $\mathrm{p}_{2}$, at the demodulator input, the output noise density, $\mathrm{P}_{6}$, at a frequency $\omega$, is due to the sum of the densities at frequencies $\left(\omega_{5}-\omega\right)$ and $\left(\omega_{5}+\omega\right)$. When, as will be assumed, $\mathrm{P}_{2}$ is constant across the input acceptance band of the demodulator, the output mean square density will be twice that due to the upper component alone. Taking into account the mean square transfer ratio of 0.5 , the resultant output noise density is:

$$
\mathrm{p}_{6}=\mathrm{p}_{2}
$$


This is an easy rule to remember, resulting from the choice of constant in Equation 3.1-3. The total output mean square noise is:

$$
\mathrm{N}_{6}^{2}=\mathrm{P}_{6}{ }^{\mathrm{B}} 6=\mathrm{P}_{2}{ }^{\mathrm{B}} 6
$$

where $B_{6}$ is the noise bandwidth of the lowpass output filter.

We need a convention for expressing the noise output in terms of the signal rating of the channel. In communications analysis, the rms full-load sine wave is usually used as a reference level. In telemetry it has been conventional to use "percent of full scale" as a measure of error, and this will be done here. We define $\mathrm{E}_{6}$ as the nominal dc output rating of the channel, knowing that the hardware may be designed to permit excursions in excess of $\mathrm{E}_{6}$. The full scale range of the channel is then $2 E_{6}$, by definition. The output noise in fraction of full scale is then:

$$
\mathrm{R}_{6}=\frac{\mathrm{N}_{6}}{2 \mathrm{E}_{6}}=\frac{\sqrt{\mathrm{P}_{2} \mathrm{~B}_{6}}}{2 \mathrm{E}_{6}}=\frac{1}{2} \sqrt{\frac{\mathrm{P}_{2} \mathrm{~B}_{6}}{\mathrm{P}_{2}}}
$$

The signal level, $E_{6}$, is related to the value of transmitter frequency deviation, $f_{d}$, due the channel input, and the noise density can be expressed in terms of equivalent modulation of the received radio signal, even though the noises are created in and after the receiver. In this way it is not necessary to specify a variety of gain factors which are variable.

In addition to the additive noise on the demodulator output, there are errors due to phase errors between the subcarrier and reference signals, caused, by phase noise in the reference generation process and by errors in tracking the time base error in magnetic tape link operation. The phase error will be composed of a steady component, $\theta_{a}$ and a fluctuating component, $\theta_{b}$. The steady component will cause a fixed error which will be corrected by the channel level control or which may be corrected by calibration. The output due to the steady component will be:

$$
e_{6}=\frac{E_{2}}{\sqrt{2}} \cos \theta_{a} \text {. }
$$

The fluctuating error due to $\theta_{b}$ will be:

$$
\theta_{b}=\frac{E_{2}}{\sqrt{2}} \cos \left(\theta_{a}+\theta_{b}\right)-\frac{E_{2}}{\sqrt{2}} \cos \theta_{a} .
$$


The output instantaneous noise as a fraction of $e_{6}$ is:

$$
\begin{aligned}
\frac{e_{b}}{e_{6}} & =\frac{\cos \left(\theta_{a}+\theta_{b}\right)}{\cos \theta_{a}}-1 \\
& \approx-\frac{1}{2} \theta_{b}^{2}-\theta_{b} \tan \theta_{a}
\end{aligned}
$$

If the fluctuating phase error is Gaussian with rms value $\phi_{b}$, the rms value of the first term in Equation 10 is $\phi_{b} 2 / \sqrt{2}$. The rms value of the second term is $\phi_{b}$ tan $\theta_{a}$. The two contributions, although functionally dependent, add powerwise. When $e_{6}$ is equal to $E_{6}$, the nominal peak channel output, the error is maximum, and its per-unit value in terms of full scale is half the value given by Equation 3.1-10. The rms error in terms of full scale is given by:

$$
\begin{aligned}
R_{6} & =\frac{r m s e_{b}}{2 E_{G}} \\
& =\frac{1}{2}\left(\phi_{b}^{4} / 2+\phi_{b}^{2} \tan ^{2} \theta_{a}\right)^{1 / 2}
\end{aligned}
$$

The rms data output error in terms of full scale channel rating is plotted in Figure 3.1-1, as a function of fixed phase error $\theta_{a}$ and rms fluctuating error $\phi_{b}$.

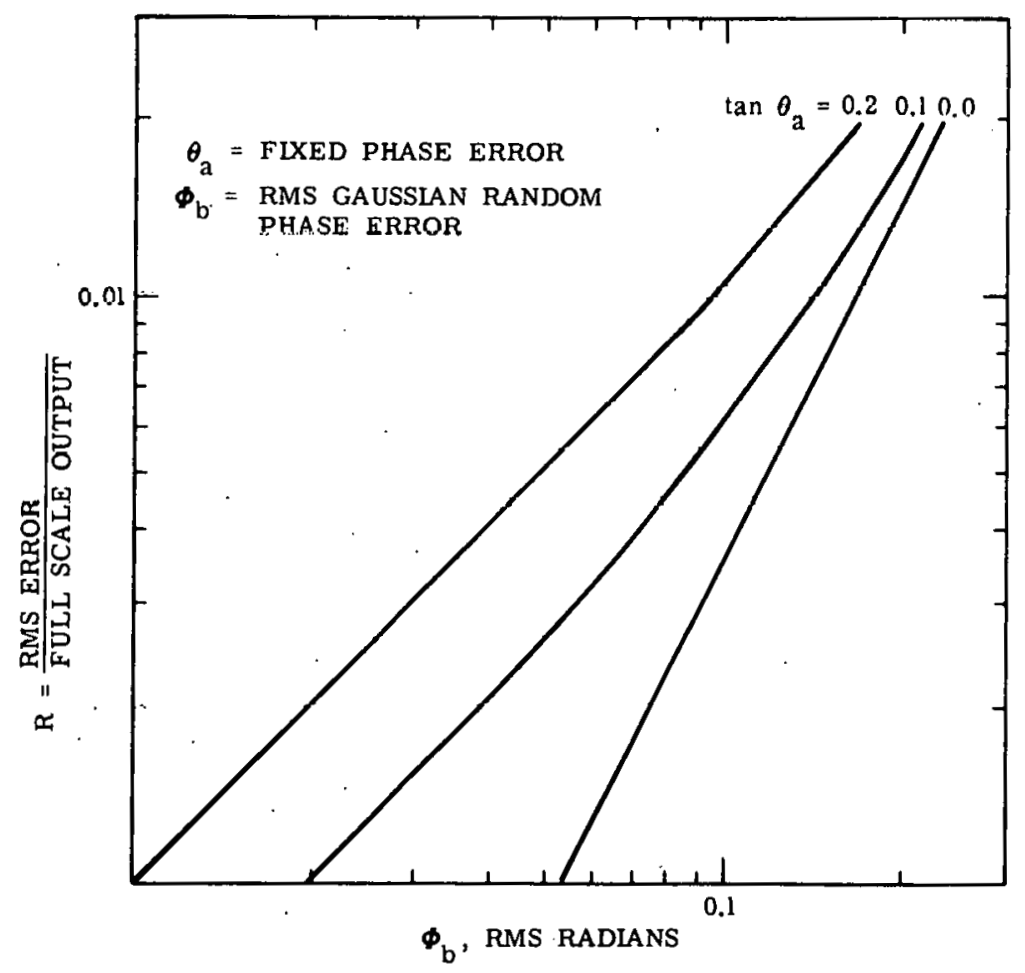

Figure 3.1-1

Demodulator output error due to fluctuating phase error. 
Symbols used in Section 3.1

${ }_{6} 6$ Demodulator output lowpass filter noise bandwidth, Hertz

$\mathrm{E}_{2} \quad$ Peak amplitude of sinusoidal subcarrier signal input to demodulator, volts. $E_{2}$ is variable, proportional to channel input.

Instantaneous subcarrier signal voltage, volts

Instantaneous demodulator reference signal voltage, volts

Peak value of $e_{5}(t)$

$3.1-2$

Demodulator output data voltage, volts

$3.1-3$

Instantaneous sinusoidal noise voltage, volts

$3.1-4$

Peak value of $e_{n}(t)$

$3.1-4$

Nominal demodulator output voltage rating, volts. $\mathrm{E}_{6}$ is one-half the full scale (peak-to-peak) rating, and corresponds to nominal subcarrier input to the demodulator

$e_{b}$ Instantaneous demodulator output error due to fluctuating phase error between subcarrier and reference inputs, volts

$\mathrm{N}_{6} \quad$ rms demodulator noise output from lowpass data filter, due to baseband noise at signal input to demodulator, volts

$\mathrm{P}_{2} \quad$ Mean square subcarrier input to demodulator, volts ${ }^{2}$, or expressed as mean square carrier deviation, Hertz ${ }^{2}$

$\mathrm{p}_{2} \quad$ Noise pqwer density at demodulator input at frequency $f_{2}$ volt $2 /$ Hertz, or expressed as equivalent carrier deviation Hertz $2 /$ Hertz

Noise power density at demodulator output due to density $\mathrm{p}_{2}$ at input, volt $2 /$ Hertz

$\mathrm{R}_{6}$ rms error on demodulator output as a fraction of full scale output

Subcarrier frequency, $2 \pi \mathrm{f}_{2}$, radian/second

$3.1-1$

Demodulator reference frequency, radian/second

$3.1-2$

Sinusoidal noise component frequency, radian/second

$3.1-4$

rus value of $\theta_{\mathrm{b}}(L)$, radians

$3.1-11$

Steady phase error between subcarrier and reference inputs to demodulator, radians

Fluctuating phase error between subcarrier and reference inputs to demodulator, radians

Phase of subcarrier input to demodulator, radians 


\subsection{Tinear Phase Lock Loop Error Trancfer}

Figure 3.2-1 defines the type of phase lock loop (PLL) being discussed. Its open-loop transfer function, $A_{1}(s)$, illustrated in Figure 3.2-2 is:

$$
A_{1}=\left(\frac{\omega_{a}}{s+\omega_{a}}\right)\left(\frac{s+\omega_{b}}{\omega_{b}}\right)\left(\frac{\omega_{c}}{s}\right)
$$

The output/input transfer ratio, the closed loop transfer function, is:

$$
A_{2}=A_{1} /\left(1+A_{1}\right)
$$

The error/input transfer ratio is:

$$
A_{3}=1 /\left(1+A_{1}\right)
$$

Figure 3.2-2 defines several characteristic parameters of the 1oop. The frequency $\omega_{c}=2 \pi \mathrm{f}_{c}$ is the product of the phase detector gain $\mathrm{K}_{1}$, the amplifier gain $\mathrm{K}_{2}$, and the VCO deviation gain $\mathrm{K}_{3}$. It is the frequency at which the upper $-6 \mathrm{~dB} /$ octave asymptote crosses the unity-gain axis. The frequency $\omega_{a}=2 \pi \mathrm{f}_{\mathrm{a}}$ is the low-frequency corner in the loop filter transfer. The frequency $\omega_{b}=2 \pi \mathrm{f}_{\mathrm{b}}$ is the high-frequency corner. The natural frequency $\omega_{n}=2 \pi f_{n}$ is where the extension of the $-12 \mathrm{~dB} /$ octave portion crosses the axis. Its value is given by:

$$
\omega_{\mathrm{n}}=\sqrt{\omega_{\mathrm{a}} \omega_{\mathrm{c}}}
$$

The damping factor, $\zeta$, depends primarily upon the open-loop gain at the frequency of the upper frequency corner. For the usual loop, the gain at the corner of the asymptotes is about $4 \zeta^{2}$. The damping factor is given by:

$$
\zeta=\frac{1}{2}\left(\frac{\omega_{\mathrm{n}}}{\omega_{\mathrm{c}}}+\frac{\omega_{\mathrm{n}}}{\omega_{\mathrm{c}}}\right) .
$$

For useful loop parameters the first term in Equation 3.2-5 is much 1arger than the second. For discussion of numerical values in what follows, a damping factor of 0.707 will be used.

Two PLL characteristics of interest are the noise bandwidth, $\mathrm{B}_{\mathrm{L}}$, and the error transfer characteristics. The former bears on the DSB demodulation phase errors due to noise, and the latter on the phase 

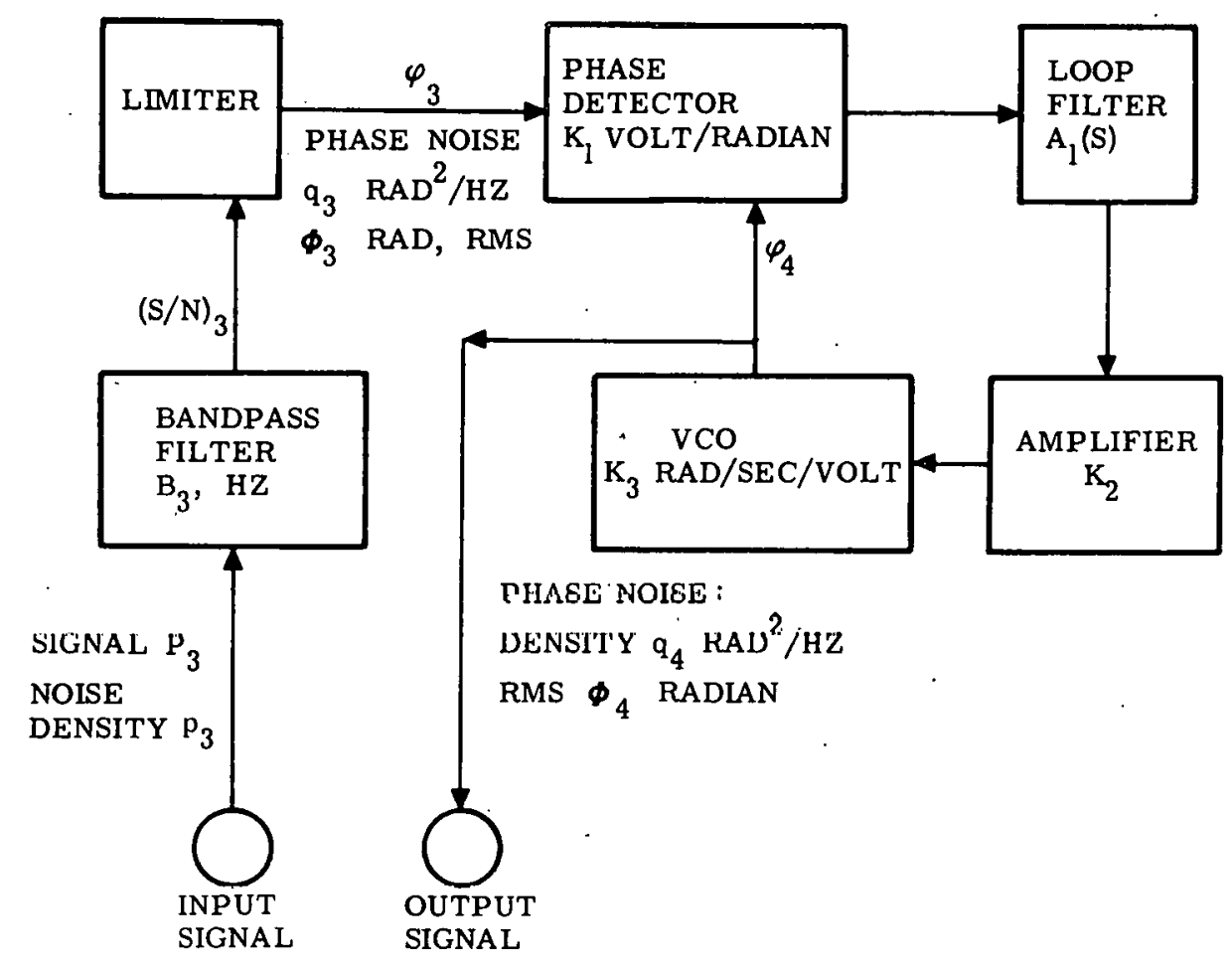

Figure 3.2-1 Phase lock loop block diagram

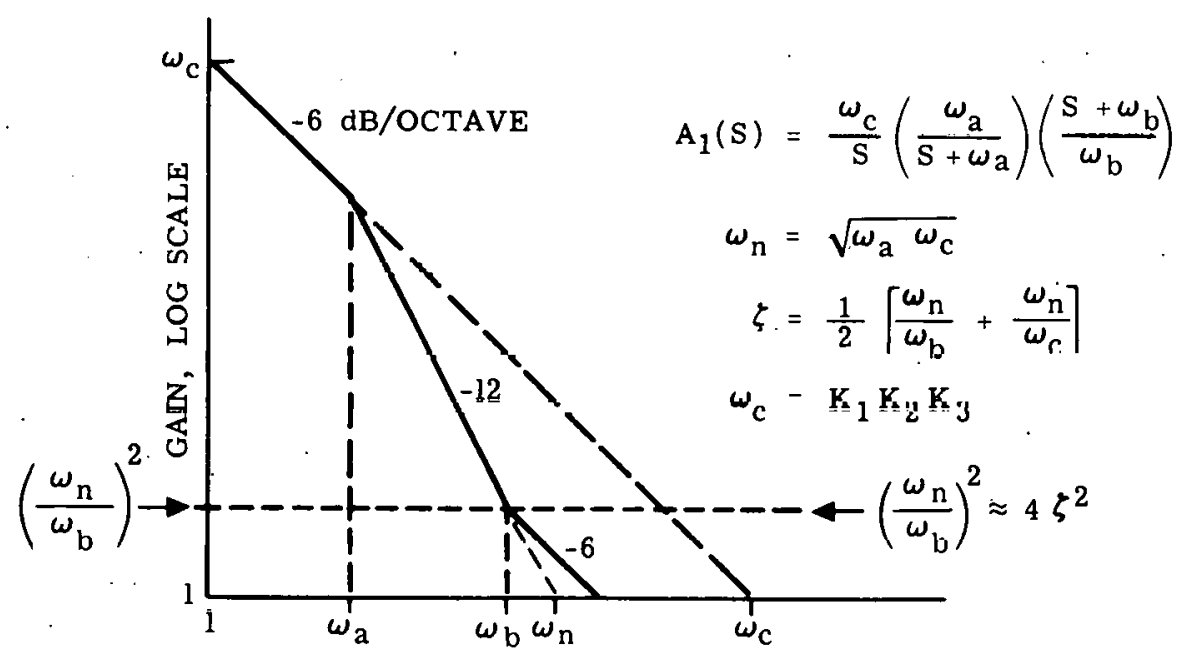

Figure 3.2-2 Open-1oop transfer function magnitude 
errors due to magnetic tape link flutter. It is desirable for the loop to ignore the noise and track the flutter.

With 0.707 damping factor the $-3 \mathrm{~dB}$ bandwidth of the closed-1oop transfer occurs at about twice the loop natural frequency. The noise bandwidth for tracking flat random noise is about $\pi / 2$ times the $-3 \mathrm{~dB}$ bandwidth. Therefore the noise bandwidth is about 3.14 times the natural frequency (both in the same units). When the input bandpass filter bandwidth is less than twice the loop noise bandwidth, $B_{L}$, the resulting phase noise filtering is determined primarily by the input filter rather than by the 1oop.

For real frequencies $(s=j \omega)$, Equation 3.2-3 gives the error transfer magnitude (error/input) as:

$$
\left|A_{3}\right|=\sqrt{\frac{\omega^{4}+\omega_{a}^{2} \omega^{2}}{\omega^{4}+\left(4 \zeta^{2}-2\right) \omega_{n}^{2} \omega^{2}+\omega_{n}^{4}}}
$$

When the damping factor is 0.707 , this simplifies to:

$$
\left|A_{3}\right|=\left(\frac{\omega}{\omega_{n}}\right)^{2} \sqrt{\frac{1+\left(\omega_{a} / \omega\right)^{2}}{1+\left(\omega / \omega_{n}\right)^{4}}}=\sqrt{\frac{1+\left(\omega_{a} / \omega\right)^{2}}{1+\left(\omega_{n} / \omega\right)^{4}}}
$$

For frequencies between $f_{a}$ and $f_{n}$ the first form of Equation 3.2-7 shows that the error ratio is approximately $\left(f / f_{n}\right)^{2}$. For frequencies above $f_{n}$, the second form shows that the error ratio is approximately unity. The maximum error in these two approximations in the neighborhood of $f_{n}$ is about $3 \mathrm{~dB}$, with the actual error being less than the approximation. 'The usefulness of these approximations in calculating. phase errors due to tape link flutter was demonstrated by M. H. Nichols. (Reference 2).

\section{Phase Errors Due to Random Noise}

Referring to the PLL of Figure 3.2-1, consider a sinusoidal signal with mean square level $\mathrm{P}_{3}$ entering the bandpass filter, accompanied by random noise with a mean square spectral density $\mathrm{P}_{3}$. If $\mathrm{P}_{3}$ is expressed in volt ${ }^{2}$ then $p_{3}$ will be in volt ${ }^{2} /$ Hertz. If the signal is obtained from an FM receiver, it will be convenient to substitute Hertz ${ }^{2}$ (of equivalent carrier deviation) for volt ${ }^{2}$ in both quantities. 
The signal-to-noise power ratio at the limiter input will be:

$$
(\mathrm{S} / \mathrm{N})_{3}^{2}=\mathrm{P}_{3} / \mathrm{P}_{3} \mathrm{~B}_{3}
$$

where $B_{3}$ is the input filter noise bandwidth in Hertz. The limiter output will be a square wave, phase modulated as a result of the input noise. When the signal-to-noise ratio is above, say, $10 \mathrm{~dB}$, there is approximately linear translation of the input noise into phase noise, with occasional bursts of decidedly nonlinear translation due to noise peaks which approach the signal level. The translation is such that nolse above the signal frequency is translated into the neighborhood of zero frequency without inversion of spectrum, and noise below the signal frequency is translated with inversion and lays on top of the noise due to the upper band.

A sinusoidal noise component of mean square value $P$ will cause a peak phase modulation of $\left(\mathrm{P} / \mathrm{P}_{3}\right)^{1 / 2}$ radians, or a mean square value of $\mathrm{P} / 2 \mathrm{P}_{3}$. The random noise is translated with the same gain, namely $1 / 2 \mathrm{P}_{3}$. If the noise density at the limiter input is symmetrical about the signal frequency, the one-sided output noise density will be twice the mean square level due to the gain, because of the contributions from above and below the signal. Therefore the output phase noise density, in $\operatorname{radian}^{2} /$ Hertz, will be twice the input density multiplied by $1 / 2 \mathrm{P}_{3}$, as follows:

$$
\mathrm{q}_{3}=\frac{\mathrm{P}_{3}}{\mathrm{P}_{3}}=\frac{1}{\mathrm{~B}_{3}(\mathrm{~S} / \mathrm{N})_{3}^{2}} \quad \operatorname{Radian}^{2} / \mathrm{H}_{2}
$$

The total mean square phase noise on the 1 imiter output is $\mathrm{q}_{3} \mathrm{~B}_{3} / 2$, because its lowpass spectral density has a noise bandwidth of $B_{3} / 2$. If the PLL noise bandwidth is much greater than $B_{3} / 2$, it will effective1y track the phase noise, giving an output mean square phase noise:

$$
\phi_{3}^{2}=\frac{\mathrm{B}_{3} \mathrm{P}_{3}}{2 \mathrm{P}_{3}}=\frac{1}{2(\mathrm{~S} / \mathrm{N})_{3}^{2}} \text { Radian }{ }^{2}
$$

When the loop noise bandwidth is much less than $B_{3} / 2$, the PLL output phase noise, mean square, is:

$$
\begin{aligned}
\phi_{4}^{2} & =\mathrm{q}_{3} \mathrm{~B}_{\mathrm{L}}=\mathrm{p}_{3} \mathrm{~B}_{\mathrm{L}} / \mathrm{P}_{3} \\
& =\frac{\mathrm{B}_{\mathrm{L}}}{(\mathrm{s} / \mathrm{N})_{3}^{2} \mathrm{~B}_{3}}
\end{aligned}
$$


where the lonp handwitith, $\mathrm{R}_{\mathrm{L}}$, is given by:

$$
B_{L}=\int_{0}^{\infty}\left|A_{2}\right|^{2} d f
$$

and $A_{2}$ is the closed-1oop output-to-input transfer ratio.

For the loop of Figure 3.2-2, with a 0.707 damping factor, the noise bandwidth is about 3.14 times $f_{n}$. The variation of noise bandwidth with loop parameters is thoroughly discussed in Reference 13.

If the PLL output is used as the demodulator-reference without frequency synthesis, the value of phase noise, $\phi_{5}$, at the demodulator is the same as $\phi_{4}$ given by equation 3.2-11. If the PLL output at frequency, $\mathrm{E}_{3}$, is used to synthesize a demodulation reference at channel frequency, $\mathrm{f}_{2}$, the phase noise at $\mathrm{f}_{2}$ will be:

$$
\phi_{5}=\left(\mathrm{f}_{2} / \mathrm{f}_{3}\right) \phi_{4}=\left(\mathrm{f}_{2} / \mathrm{f}_{3}\right) \sqrt{\mathrm{P}_{3} \mathrm{~B}_{\mathrm{L}} / \mathrm{P}_{3}}
$$

With independent subcarriers the PLL tracks the modulated subcarrier at frequency $f_{2}$, so that $f_{2}$ is substituted for the pilot frequency $\mathrm{f}_{3}$. The noise density $\mathrm{P}_{2}$ at the subcarrier frequency is the value to be used for $\mathrm{p}_{3}$. When the subcarrier is steady (with dc data into the channel modulator) the mean square subcarrier level, $\mathrm{P}_{2}$, is used for $\mathrm{P}_{3}$.

With independent subcarriers there is usually a channel pilot signal added to the data signal, so that even with de data the value of $\mathrm{P}_{2}$ is modulated. In this case there is no known general solution for the value of phase nolse. When the pilot peak voltage is small compared to the data voltage, and the noise in demodulator input filter bandwidth $B_{2}$ is small compared to $P_{3}$, equation $3 \cdot 2-11$ is useful. As the data voltage is lowered, or variable, the value of rms phase noise is found by experiment. An actual phase noise measurement is undoubtedly cheaper, if less impressive, than a digital computer solution.

\section{Phase Errors Due to Tape Link Flutter}

Equation 3.2-7 gives the PLL tracking error resulting from a sinusoidal phase variation of the input signal. When a signal is phasemodulated by tape speed variations, the power spectrum of the tape link 
time base error (TBE) is multiplied by the PLL error transfer ratio squared, and integrated to obtain the mean square untracked TBE. The rms untracked TBE multiplied by the signal frequency gives the rms phase error between the PLL oscillator output phase and the signal input phase. Reference 2 demonstrates an example of this calculation in the case where the TBE power spectrum is approximated by:

$$
\mathrm{z}^{2}=10^{-7} / 4 \mathrm{f}^{4} \quad \text { second } 2 / \mathrm{Hz}
$$

in which case the calculation is easy. In the following development this procedure is generalized to produce a chart giving untracked TBE for any spectrum of the form:

$$
\mathrm{z}^{2}=\mathrm{k}_{\mathrm{z}}^{2} \mathrm{f}^{\mathrm{n}}
$$

when $\mathfrak{n}$ lies in the range between -1.5 and $-4: 0$.

For frequencies below $f_{\mathfrak{n}}$ the error transfer ratio from Equation 3.2-7 is approcimately $\left(f / f_{n}\right)^{2}$ and the untracked mean square TBE is:

$$
\begin{aligned}
T^{2} & =\int_{f}^{f_{n}}\left(f / f_{n}\right)^{4} k_{z}{ }^{2} f^{n} d f \\
& =\frac{k_{z}{ }^{2}}{n+5} f_{n}^{n+1}\left[1-\left(\frac{f}{f_{n}}\right)^{n+5}\right] \approx \frac{k_{z}^{2}}{n+5} f_{n}^{n+1}
\end{aligned}
$$

For a variety of exponents $(n)$ and reasonable integration limits ( $f$ ) the second term in Equation 3.2-16 may be neglected as shown.

For frequencies above $f_{n}$ the error transfer ratio is unity, and the resulting mean square untracked TBE is:

$$
\begin{aligned}
T^{2} & =\int_{f_{n}}^{f} k_{z}{ }^{2} f^{n} d f \\
& =\frac{-k_{z}^{2}}{n+1} f_{n}{ }^{n+1}\left[1-\left(\frac{f}{f_{n}}\right)^{n+1}\right] \approx \frac{-k_{z}^{2}}{n+1} f_{n}^{n+1}
\end{aligned}
$$

where again the second term may be neglected for reasonable values of the upper limit of integration, $f$, and for $n+1$ somewhat less than zero. Summing the contributions from below and above $f_{n}$ gives the following simple relationship, which is plotted as $T / K_{z}$ in Figure 3.2-3:

$$
T^{2}=\frac{-4 k_{z}^{2}}{(n+5)(n+1)} f_{n}^{n+1} \text { second }{ }^{2}
$$




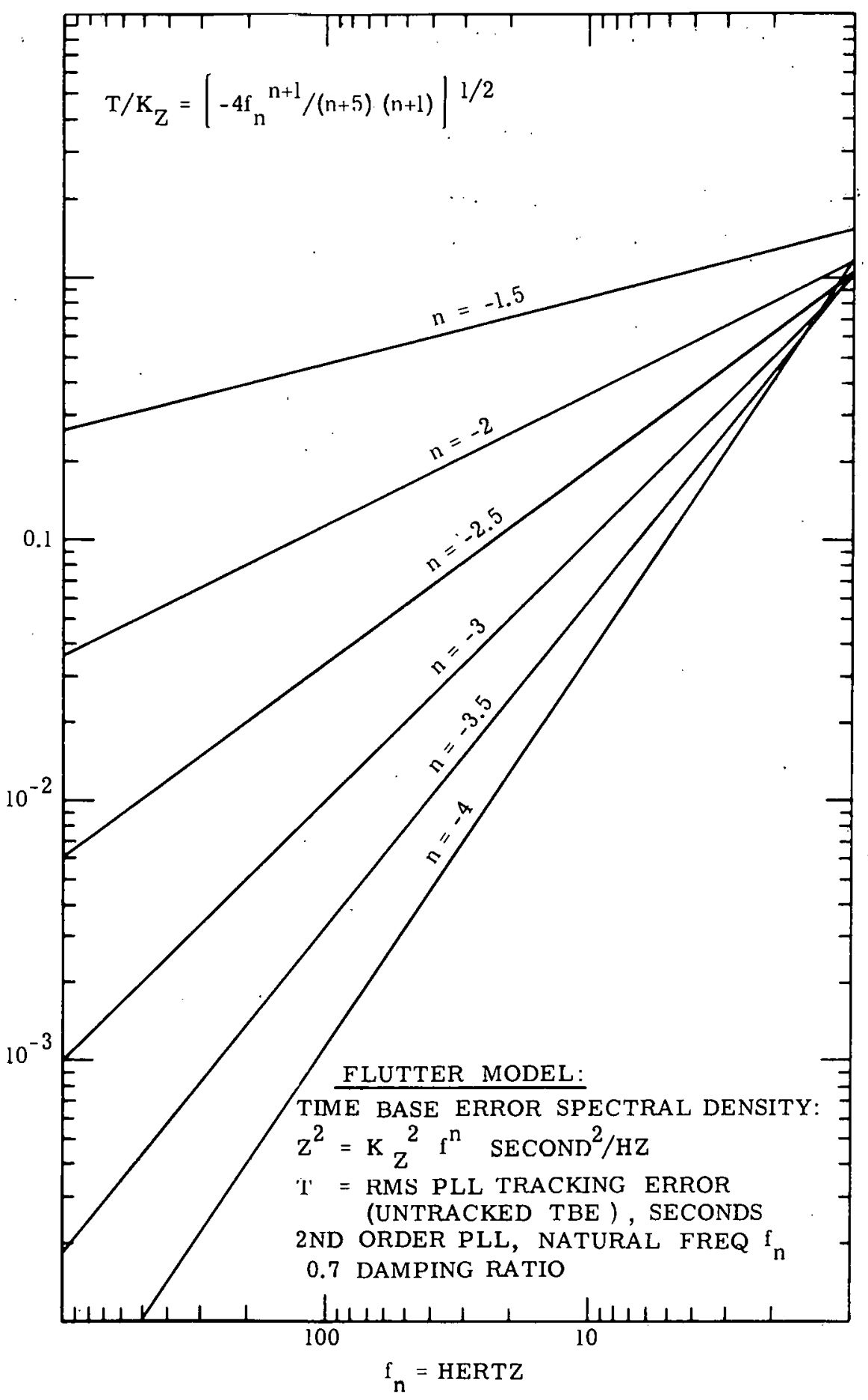

Figure 3.2-3 Phase lock loop tracking error due to flutter 
For a pilot signal at a frequency $f_{3}$, the rms phase error in tracking flutter is:

$$
\phi_{4}=2 \pi \mathrm{f}_{3} \mathrm{~T} \quad \text { rms radians (3.2-19) }
$$

A demodulator reference signal synthesized from the pilot signal at $f_{3}$ will have an rms phase error, $\phi_{5}$, given by:

$$
\begin{aligned}
\phi_{5} & =\left(f_{2} / f_{3}\right) \phi_{4}=2 \pi f_{2} T \\
& -2 \pi f_{2} k_{z}\left[\frac{-f_{1} f_{n 1}^{n+1}}{(n+5)(n+1)}\right]^{1 / 2}
\end{aligned}
$$

where the quantity in brackets is gotten from Figure 3.2-3. 
Symbols Used in Section 3.2

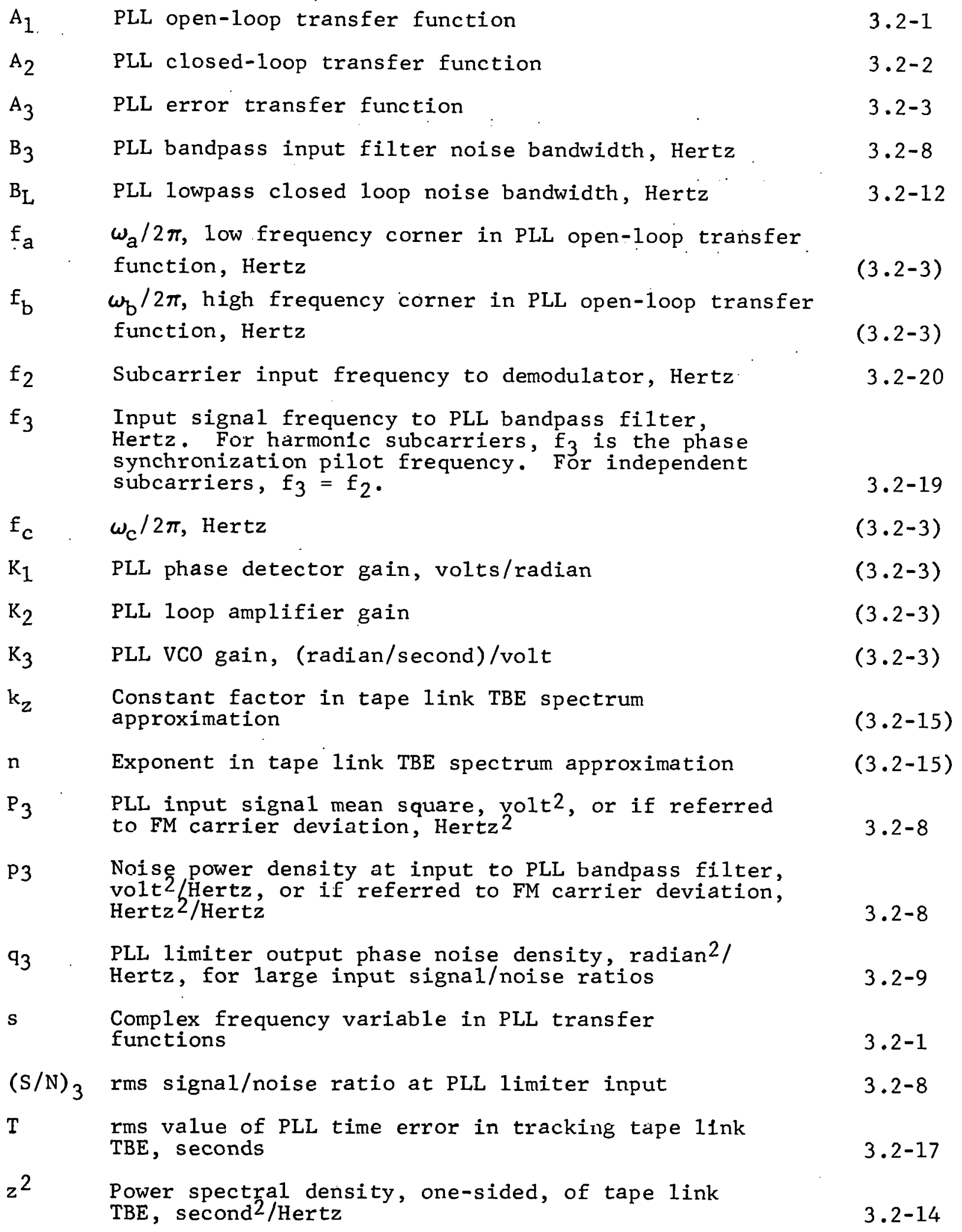




$\begin{array}{lll}\omega_{\mathrm{a}} & 2 \pi \mathrm{f}_{\mathrm{a}}, \text { radians/second } & 3.2-1 \\ \omega_{\mathrm{b}} & 2 \pi \mathrm{f}_{\mathrm{b}}, \text { radians/second } & 3.2-1 \\ \omega_{\mathrm{c}} & \mathrm{K}_{1} \mathrm{~K}_{2} \mathrm{~K}_{3} \text { radians/second } & 3.2-1 \\ \omega_{\mathrm{n}} & \text { PLL natural frequency, radians/second } \\ \phi_{3} & \begin{array}{l}\text { PLL limiter output rms phase noise, radians, for } \\ \text { large input signal/noise ratio }\end{array} \\ \phi_{4} & \begin{array}{l}\text { PLL output rms phase noise, radians, for large } \\ \text { limiter input signal/noise ratio }\end{array} \\ \phi_{5} & \begin{array}{l}\text { Demodulator reference input phase noise, radians, } \\ \text { for harmonic oubcarricro }\end{array} \\ \zeta & \text { PLL damping factor }\end{array}$




\subsection{Baseband Leve1 Control Noise Transfer}

The pilot signal for the baseband level control (BLC) is accompanied by noise from a variety of sources. The BLC 1oop attempts to keep the output of the BLC pilot demodulator at a constant level, and in so doing tracks that portion of the output which falls within the loop noise bandwidth. The resulting gain modulation has a per unit value equal to the ratio of noise to de signal output from the pilot demodulator, considering only that noise falling within the acceptance band of the loop, which we will call $\mathrm{B}_{\mathrm{b}}$, the one-sided noise bandwidth of the BLC 1oop.

We assume that the pilot demodulator has the same transfer function as the subcarrier demodulators, except that it is not phase sensitive. This phase sensitivity is of no consequence because the pilot amplitude is only of one polarity. When the pilot signal-to-noise ratio is much larger than unity (as is necessary for useful operation), the noise density transfer is linear and of the same value as for the subcarrier demodulator. The pilot signal in the baseband is:

$$
e_{3}=E_{3} \sin \omega_{3} t
$$

The demodulated pilot signal dc value, $e_{b}$, is:

$$
e_{b}=E_{3} / \sqrt{2}
$$

For a flat mean square noise density, $P_{3}$, the one-sided output noise density from the pilot demodulator, $\mathrm{p}_{\mathrm{b}}$, is:

$$
\mathrm{p}_{\mathrm{b}}=\mathrm{p}_{3}
$$

The mean square noise tracked by the BLC 10op is:

$$
\mathrm{N}_{\mathrm{b}}^{2}=\mathrm{P}_{\mathrm{b}} \mathrm{B}_{\mathrm{b}}=\mathrm{p}_{3} \mathrm{~B}_{\mathrm{b}}
$$

The per-unit gain modulation is $\mathrm{N}_{\mathrm{b}}$ divided by the $\mathrm{dc}$ level, $\mathrm{e}_{\mathrm{b}}$, of the pilot demodulator output:

$$
\frac{\mathrm{N}_{\mathrm{b}}}{\mathrm{e}}=\frac{\sqrt{\mathrm{P}_{3} \mathrm{~B}_{\mathrm{b}}}}{\mathrm{E}_{3} / \sqrt{2}}=\sqrt{\frac{\mathrm{P}_{3} \mathrm{~B}_{\mathrm{b}}}{\mathrm{P}_{3}}}
$$

where $\mathrm{P}_{3}$ is the mean square baseband pilot signal level in the same units as $\mathrm{P}_{3} \mathrm{~B}_{\mathrm{b}}$. 
The gain modulation modulates all of the subcarrier signals which pass through the BLC, and the same fractional modulation appears on the outputs of all the subcarrier demodulators. The noise on the subcarrier demodulator output, with signal output level, $e_{6}$, is:

$$
\mathrm{N}_{6}=\mathrm{e}_{6} \sqrt{\frac{\mathrm{P}_{3} \mathrm{~B}_{\mathrm{b}}}{\mathrm{P}_{3}}}
$$

The ratio, $R_{6}$, of noise, $N_{6}$, to full-scale signal range, $2 E_{6}$, is:

$$
R_{6}=\frac{N_{6}}{2 E_{6}}=\frac{\mathrm{e}_{6}}{2 \mathrm{E}_{6}} \sqrt{\frac{\mathrm{P}_{3} \mathrm{~B}_{\mathrm{b}}}{\mathrm{P}_{3}}}
$$


Symbols Used in Section 3.3

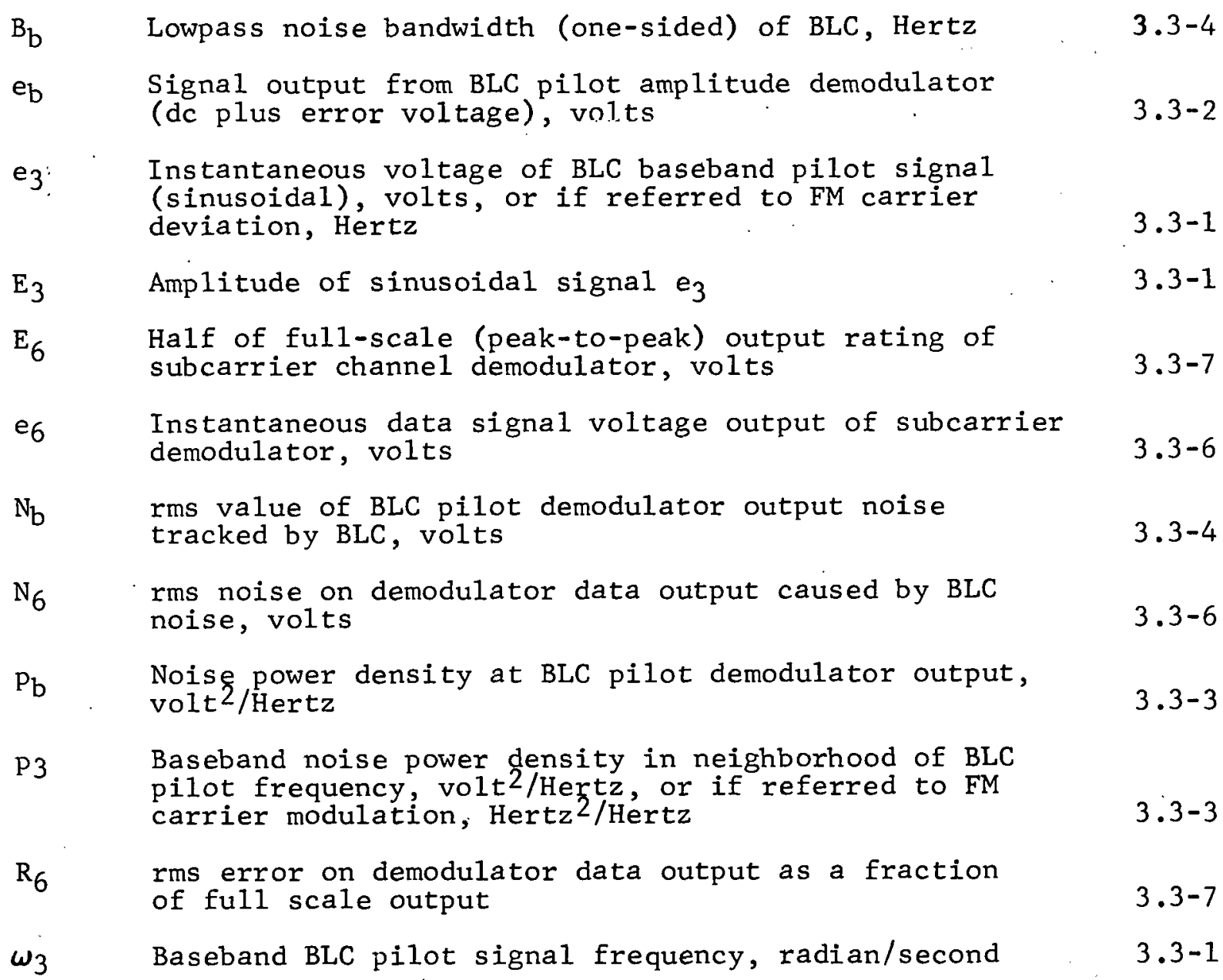




\subsection{Subcarrier Synchronization}

\section{Harmonic Subcarrier Synchronization}

Demodulation reference subcarrier signals are synthesized from an oscillator phase-locked to the phase synchronization pilot whose frequency is $\mathrm{F}_{3}$. The reference subcarrier frequencies and $\mathrm{F}_{3}$ are integer multiples of a base frequency, $F_{b}$. At a time $t_{0}$ all of the synthesized reference signals have a specified phase relationship, which for discussion will be taken as all references having a positive-going zero crossing. The base frequency signal is also generated in the synthesizer, and has a positive-going zero crossing at $t_{0}$. The synch pilot frequency is $n_{b}$, where $\mathrm{n}$ is an integer. A phase reset pilot signal is also received, which for discussion we assume also has a frequency $F_{b}$. The basefrequency signal derived from the synch pilot may initially have any of $n$ different phases with respect to the reset pilot, separated by $360 / \mathrm{n}$ degrees.

In Figure 3.4-1, to is identified with one cycle of the phase synch pilot, and the resulting base frequency timing shown on the second line. Also shown dotted on the second line is the timing of the base frequency signal in another possib le initial state of the synthesizer. The third line shows the resulting demodulator reference timing for these two states, for comparison with an incoming subcarrier signal on the fourth line. Obviously the second state is undesirable. This undesired state is eliminated by comparing the base-frequency signal and reset pilot phases and jogging the synthesizer until the desired state is obtained.

The reset pilot, as illustrated on the fifth line, is subjected to noises and phase-shifts in transmission, filtering, etc., so that exact phase coincidence is not obtained. The allowable phase tolerance on the reset pilot will now be examined.

If error-free phase comparison could be made, it would be possible to identify the correct cycle of synch pilot frequency with $t_{0}$ provided that the timing error on the reset pilot were slightly less than $t_{1}$, which is half a period of the synch pilot. Allowing for some error in these circuits, we see that the allowable time error, $t_{4}$, in the reset pilot is 
something less than half of $t_{3}$, the synch pilot period. The allowable phase error is given by:

$$
\begin{array}{ll}
\alpha=360 t_{4} F_{b} & \text { Degrees } \\
\alpha<360 \frac{t_{3}}{2} F_{b}=\frac{180}{n} & \text { Degrees }
\end{array}
$$

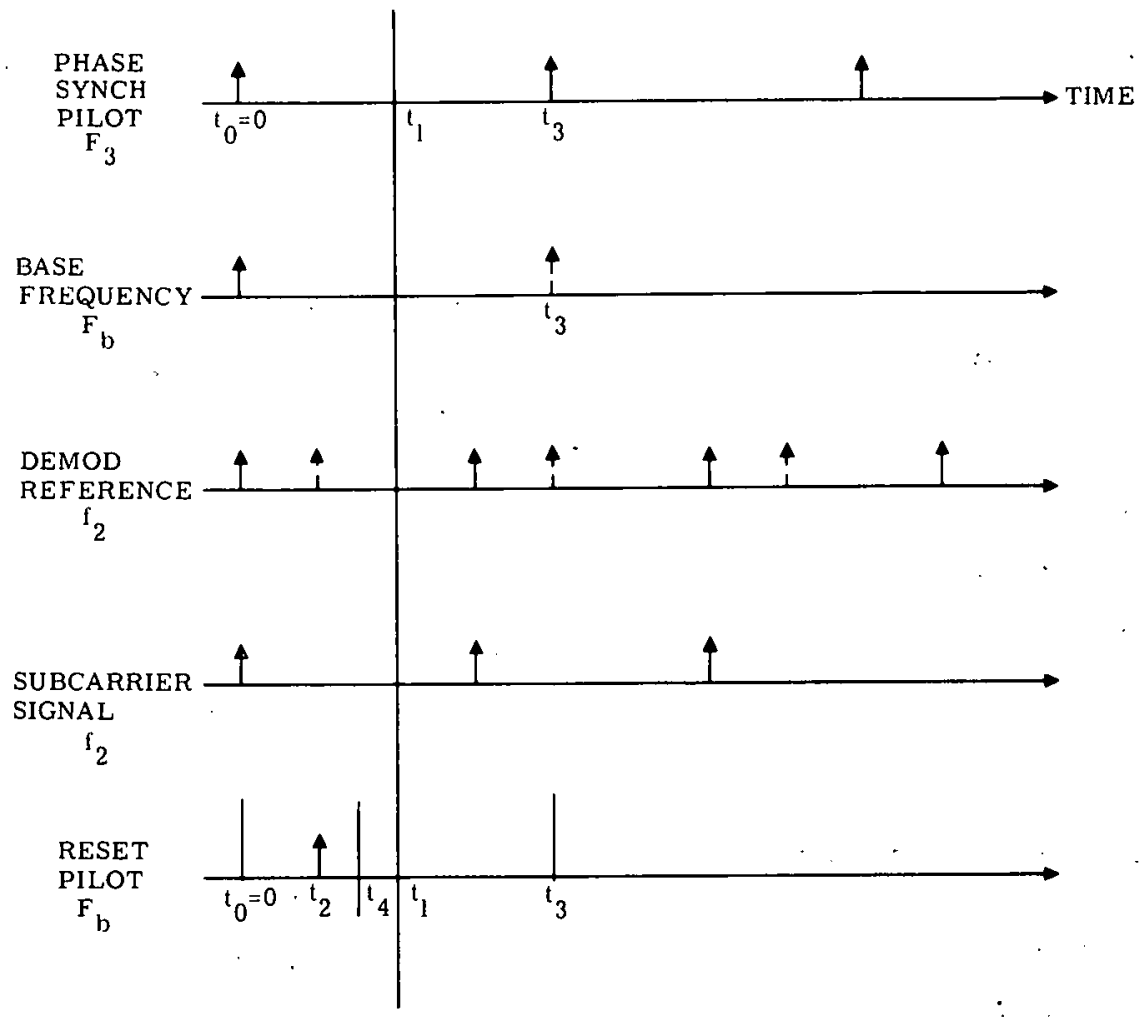

Figure 3.4-1 Harmonic subcarrier synchronization timing

It has been suggested that the factor $n$ be restricted to integer powers of two, in which case the reset pilot frequency may be any odd multiple of the base frequency (in principle). It can be shown that if the reset pilot frequency is $\mathrm{mF}_{b}$ the allowable time error on the reset pilot becomes $44 / \mathrm{m}$, resulting in the same value for allowable phase - error as given in Equation 3.4-2.

The phase error will be the sum of steady phase shifts and fluctuating phase errors. The allowable value of $t_{4}$ must also take into account errors in handling the synchronization pilot (steady and 
fluctuating) and in synthesizing the base frequency signal, as well as errors in the phase comparison and decision circuits used in the , ambiguity-resolution function.

For the present work a synch pilot at $64 \mathrm{kHz}$ will be assumed, and a reset pilot at a base frequency of $4 \mathrm{kHz}$, giving $\mathrm{n}$ a value of 16 . The resulting value of $180 / \mathrm{n}$ is 11.25 degrees (corresponding to 7.8 microseconds) of the $4 \mathrm{kHz}$ signal phase. When all the contributions to phase error are eventually sized up, the total tolerance above is not expected to be easy to meet.

\section{Independent Subcarrier Synchronization}

The demodumlation reference signal for each channel is derived from the modulated subcarrier signal for each channel. The signal, at nominal frequency $f_{2}$, is modulated in amplitude in proportion to the sum of the data signal input and the channel pilot. The polarity of the amplitude can be negative as well as positive. The amplitude and polarity variations make the PLL task more difficult than would be the case for a steady sinusoidal signal.

The subcarrier signal (and noise) is separated from the baseband signal by a filter of bandwidth $\mathrm{B}_{2}$, whose output enters a limiter followed by a frequency doubler. The phase lock loop operates on the square-wave output of the doubler, and has a phase detector whose lowfrequency output is a linear function of phase error (as contrasted to a. multiplier with sinusoidal inputs assumed by most analysts). Other models and mechanizations are possible, but this is the model used in this discussion.

The phase detector operates at the double frequency, $2 \mathrm{f}_{2}$. The phase noise at $2 f_{2}$ is twice that at the limiter output, whether due to noise or to tape time base error. A phase-shifted version of the PLL output at $2 f_{2}$ is scaled in frequency to $f_{2}$ and used as the demodulator reference. Its phase noise is one-half the PLL phase noise, but not the same as the limiter output phase noise because of the PLL characteristics. When the PLL is operating in its linear range (no saturation or excessive phase detector error) it can be analyzed as a servo with the same loop parameters operating at frequency $f_{2}$ as is done in Section 3.2 . 
We will proceed to estimate the conditions which will cause loss of lock in the phase lock loop, which we will call "unlock" for short. A general analysis for the model including the channel pilot is not available, so unlock conditions will be estimated on the basis of published data on other models. The work of R. W. Sanneman and J. R. Rowbotham, particularly Figure 12 of Reference 14, gives the mean time to unlock in terms of the rms phase variation of the PLL vco and the natural frequency of a loop having a damping factor equal to 0.707 . Using the relationship of Equation 3.2-10, F. M. Gardner (Reference 13, Figure 3-2) has noticed an exponential relationship between mean time to unlock from Reference 14 and input signal-to-noise ratio referred to a bandwidth $2 \mathrm{~B}_{\mathrm{L}}$, where $\mathrm{B}_{\mathrm{L}}$ is the one-sided niose bandwidth of the loop. We will extrapolate this relationship to values of mean time which are more tolerable in practice than those of Reference 14. Figure 3.4-2 shows the extrapolation of Gardner's relationship, with the solid portion of the curve showing the range of the Sanneman and Rowbotham data. It is comforting to notice that the curve is very steep, so that a large error in mean time to unlock corresponds to a relatively small error in input signal-to-noise ratio and rms phase noise.

In the work of this report, we use a loop natural frequency of $150 \mathrm{~Hz}$, which for a value of $\mathrm{T}_{\mathrm{a}} \omega_{\mathrm{n}}$ of $10^{5}$ gives a mean time to unlock, $\mathrm{T}_{\mathrm{a}}$ of 106 seconds. The corresponding value of PLL rms phase noise is 0.378 radians. This must be evaluated at the phase detector input at $2 f_{2}$, so the corresponding value at $f_{2}$ is half of this, or 0.189 radians. Calculations of phase error due to radio link noise and distortion are carried out in Table 3.4-1 for the $96 \mathrm{kHz}$ subcarrier channel. When the test channel signal is the nominal rated dc value, and the other channels are loaded with the radom data model, the rms phase noise at $f_{2}$ is 0.0295 radians. When the dc channel input is lowered to 0.156 of the rated input, the calculated phase noise increases to the value 0.189 above, and unlocks are estimated with $\mathrm{T}_{\mathrm{a}}$ equal to 106 seconds.

This estimate ignores the effect of the channel pilot signal (with peak value 0.2 of the rated $\mathrm{dc}$ ) on the unlocks, and the effect of the limiter input signal-to-noise ratio on the loop signal-to-noise ratio. Therefore the value of 0.156 rated dc may be in error a $d B$ or so. A1so ignored is the increase in subcarrier due to transmitter modulation control which occurs when the test channel input is decreased. 


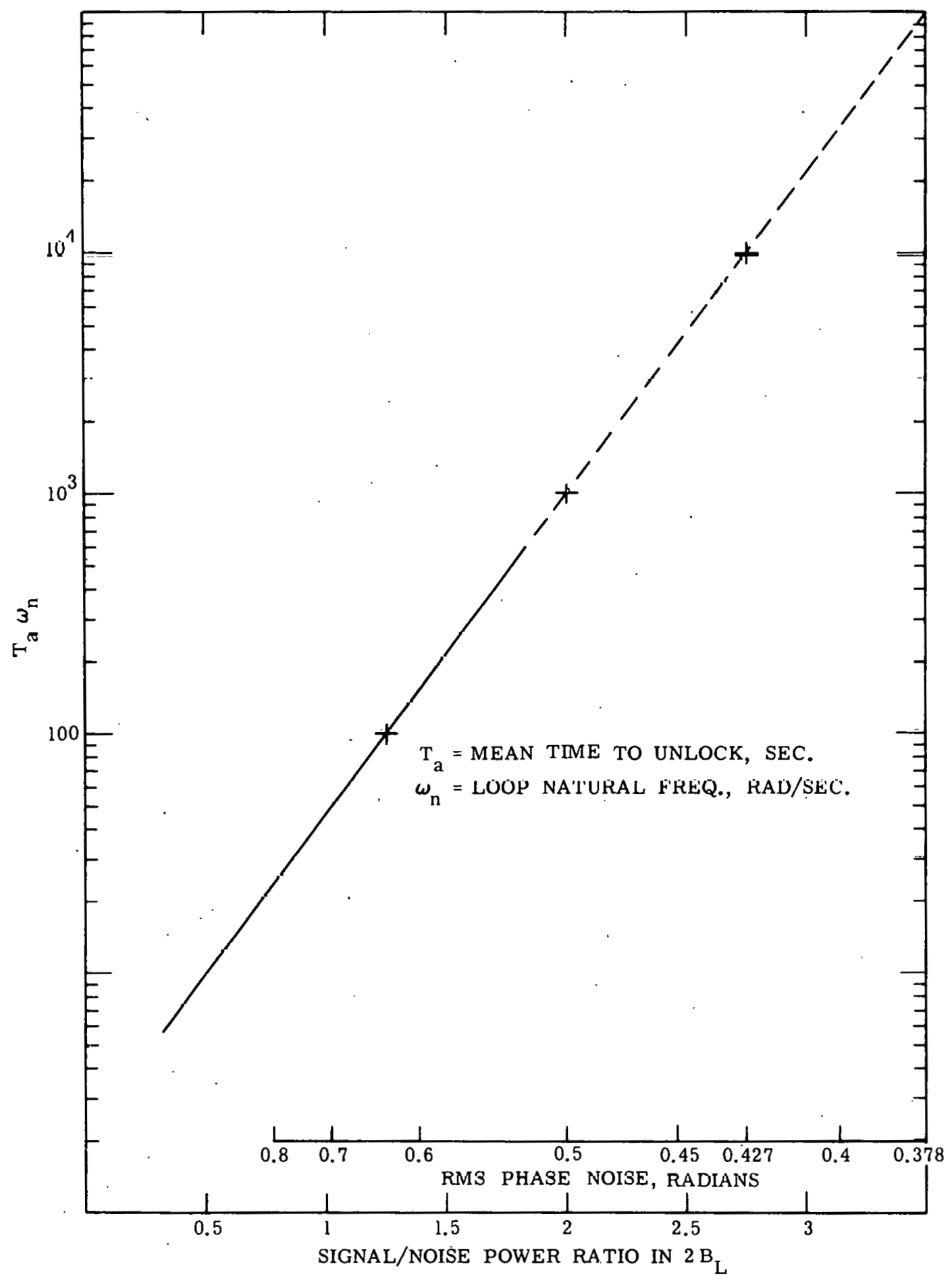

Figure 3.4-2 Mean time to unlock for PLL 
Tab1e $3.4-1$

Calculation: PLL dropout due to radio link noise independent subcarrier

Test Channel Frequency

Test Channel Nomina1 dc output

$$
\begin{aligned}
\mathrm{f}_{2} & =96 \mathrm{kHz} \\
\mathrm{E}_{6} & =5 \text { volts } \\
\sqrt{\mathrm{P}_{2}} & =25.6 \mathrm{kHz} \\
(\mathrm{S} / \mathrm{N})_{1} & =12 \mathrm{~dB} \\
\mathrm{f}_{\mathrm{n}} & =150 \mathrm{~Hz} \\
\mathrm{~B}_{\mathrm{L}} & =471 \mathrm{~Hz}
\end{aligned}
$$

Test Channe1 Nomina1 Carrier Deviation$$
\text { IF Carrier/Noise Ratio }
$$

PLL Natural Frequency, $\omega_{n} / 2 \pi$

Loop Noise Bandwidth $=\pi f_{n}$

PLL phase error due to radio link noise and distortion

$$
\begin{aligned}
& \text { Baseband noise at } \mathrm{f}_{2} \quad 1090 \quad \mathrm{~Hz}^{2} / \mathrm{Hz} \\
& \text { Baseband distortion at } \mathrm{f}_{2} \quad 120 \quad \mathrm{~Hz}^{2} / \mathrm{Hz} \\
& \text { Total } \mathrm{P}_{2}=1210 \mathrm{~Hz} / \mathrm{Hz} \\
& \text { Phase error at } \mathrm{f}_{2} \text { : } \\
& \phi_{4}^{2}=\mathrm{P}_{2} \mathrm{~B}_{\mathrm{L}} / \mathrm{P}_{2} \text {. (From equation 3.2-11) } \\
& =(1210)(471) /(25,600)^{2}=870 \times 10^{-6} \text { Radian }^{2} \\
& \phi_{4}=0.0295 \text { Radian, rms } \\
& 2 \phi_{4}=0.0590 \text { Radian, rms }
\end{aligned}
$$

For $\mathrm{T}_{\mathrm{av}} \omega_{\mathrm{n}}=10^{5}\left(\mathrm{~T}_{\mathrm{av}}=106\right.$ seconds $)$ the value of phase error at $2 \mathrm{f}_{2}$ (from Figure $3.4-2$ ) is

$$
2 \phi_{4}=0.378, \phi_{4}=0.189
$$

DC data level corresponding to $\mathrm{T}_{\mathrm{av}}=106$ seconds

$$
e_{6}=E_{6}(0.0590) /(0.378)=0.156 E_{6}=0.78 \text { volts }
$$

This calculation neglects the effect of the CLC pilot and the deviation increase due to TMC as $e_{6}$ is lowered from the nominal $E_{6}$. It also ignores threshold effects due to $(S / N)_{2}$. 
The important thing about this estimate is that it gives a value in the interesting range of operating conditions without considering any of the following contributions to unlock:

a. Saturation in any of the loop electronics other than the phase detector

b. Static phase errors in the loop

c. Additional phase errors due to tape link flutter

It is interesting to notice that unlock errors are not displayed in the data of the tests of Reference 1 , but did appear in the data of Reference 12. The conditions of the latter tests are not as well documented, so the reasons for the differences are not known.

We do not have an analysis for PLL unlock due to tape flutter. There is some level of phase error in the PLL tracking a flutter-modulated signal which will cause unlock to occur, and this level will be taken intuitively as 0.38 radians, the value of phase noise used for PLL error due to flat random noise on the subcarrier. These are not equivalent phenomena, however, because one (flutter) is the untracked modulation, whereas the other quantity is the noise which is tracked. The intuitive value applies to the frequency $2 f_{2}$, and corresponds to 0.19 radians rms at the input frequency $f_{2}$. In addition, any phase error due to flutter adds to the loop error due to noise and restricts the range of signa1to-noise ratios tolerable without unlock.

Another function necessary in phase synchronization with independent subcarriers is the phase reset, or ambiguity resolution. The output at $\mathrm{f}_{2}$ : may lock in either of two states, 180 degrees apart. The undesired state results in inverted voltage output from the demodulator. The ambiguity is resolved by comparing the phase of the channel pilot with that of the baseband pilot having the same frequency. The level of both of these pilots is assumed to be controlled by the channel and baseband level controls. In the present equipment at Sandia, this is accomplished by subtracting the two pilot signals and fogging the state of the frequency scaler whenever the difference exceeds a preset level. It does not appear to have been mentioned previously that this difference is sensitive to phase errors. These errors may be adjusted to a small value in the multiplex/demultiplex equipment. The communication link in between introduces additional errors. The only one that appears significant will be due to postdetection recording tape links. A phase 
shift of 30 degrees at the baseband pilot frequency will cause the difference between the two pilot signals to be about half the voltage of each pilot, restricting the useable operating range of the phase reset function, which is understood to be normally set' to trigger at the $-6 \mathrm{~dB}$ level. It appears that a higher threshold setting would accommodate some baseband phase shift. It is also possible to equalize the phase shift of the tape recorder links. 
Symbols Used in Section 3.4 $\mathrm{B}_{2} \quad \begin{aligned} & \text { Noise bandwidth of subcarrier demodulator bandpass } \\ & \text { input filter, Hertz }\end{aligned}$

B PLL lowpass closed-loop noise bandwidth, one-sided, Hertz

$\mathrm{F}_{3} \quad$ Frequency of phase synchronization baseband pilot, Hertz

Fig. $3 \cdot 4-1$

$F_{b} \quad$ Base frequency in harmonic subcarrier method, Hertz. The frequencies of the phase synch pilot, phase rèset pilot, ánd àll subcărrierś àre integer multiples of $F_{b}$.

Fig. $3.4-1$

$\mathrm{f}_{2} \quad$ Frequency of subcarrier signal input to demodulator, Hertz

Fig. 3 4-1

m An integer. In the harmonic subcarrier method, the phase reset pilot frequency is $\mathrm{mF}_{b}$

$\mathrm{n}$ An integer. In the harmonic subcarrier method, the phase synch pilot frequency is $\mathrm{nF}_{\mathrm{b}}$

$t_{0}$ In the harmonic subcarrier method, the time at which all pilots and subcarriers pass simultaneously through specified phase angles (such as positive-going zero crossings), seconds

Fig. $3 \cdot 4-1$

$t_{1} \quad$ Assuming $t_{0}=0, t_{1}$ is one half period of the phase synch pilot signal, seconds

Fig · $3 \cdot 4-1$

$t_{3} \quad$ Assuming $t_{0}=0, t_{3}$ is the phase synch pilot period, seconds

Fig. $3.4-1$

$t_{4}$ The maximum time error permissible in the reset pilot signal at the point of utilization in the synthesizer reset function, seconds

$t_{a}$ Mean time to loss of lock for phase lock loop, sernnis

$\omega_{n} \quad$ Natural frequency of phase lock 1oop, radian/second 


\subsection{Demodulator Reference Phase Frrors}

The steady (slowly varying) phase shifts contributing to phase error between the demodulator signal input and the subcarrier signal input are not well documented and further measurements will be needed to obtain the proper values. As can be seen from Figure 3.1-1, they have a substantial effect on the errors caused by the fluctuating component of phase error due to noise and flutter. Some of the steady errors can be removed by adjustments, leaving those errors which are dependent on the environmental variations and maintenance schedule (see, for example, Reference 12).

With independent subcarriers the bandpass filters in the airborne and ground equipment do not introduce phase errors for steady dc data signal inputs. Errors will exist for the high-frequency data components due to time delay variation across the pass band of these filters. The significance of this effect has not been evaluated but is thought to be sma11 compared to the errors due to the frequency response of the data output filters.

The demodulator reference synchronization method for independent subcarriers has been said to be free of phase errors. It is of interest to identify sources of phase error in this method even though no test data is available on their size. After leaving the demodulator bandpass input filter, the signal traverses two paths to the multiplier. The subcarrier signal path appears to be error-free. The synchronization path begins with a limiter whose output zero-crossings times depend upon delay, hysteresis, and bias levels, as well as any leakage of coherent signals into its input. Following the limiter is a trigger and oneshot multivibrator used to create the double-frequency signal for the phase lock loop. Phase shifts are caused by signal and bias level variations, by pulse length variations, and by pulse shape variations. The PLL phase detector is sensitive to pulse shape. The PLL drift is otherwise very smal1. Each of these effects is probably small and could be compensated by adjustment. A figure of 5.7 degrees is used in calculations in this report for the collection of these errors. The actual error will probably be smaller than this figure, but not zero.

The harmonic subcarrier method is subject to the effects of several sources of steady phase error which are not negligible. They consist mainly of the subcarrier synthesizers, the subcarrier filters, the 
radio link, and the magnetic tape link. Estimates of the phase error contributions for the link models and the harmonic subcarrier modification of Sandia's DSB equipment are listed in Table 3.5-1 as they affect the $16 \mathrm{kHz}$ and the $96 \mathrm{kHz}$ channels.

The variation of reference signal phases in the airborne and ground synthesizers is not known. The frequency counter delay tolerance is reported to be 40 nanoseconds, and the output amplifier delay to be 100 nanoseconds. The effects are assumed to be adjusted to zero in the overall system, leaving variation with time and temperature. For purposes of calculation the variation in each synthesizer is estimater at 30 nanoseconds, independently on each reference and pilot signal output. The $64 \mathrm{kHz}$ pilot phase variation is multiplied by the ratio of subcarrierto-pilot frequencies $\left(f_{2} / f_{3}\right)$ in calculating its effect at the subcarrier demodulator. The same factor applies to f1.1. pilot-frequency phase arrors, both steady and fluctuating.

The phase shift of the subcarrier and pilot signals due to bandpass filters is a function of the design, quality control, and environmental conditions, and the tolerances to be achieved in the Sandia equipment were not yet known. The phase shift can be inferred from the filters used in the independent subcarrier equipment. The channel pilot sidebands at $1.5 \mathrm{kHz}$ from the subcarrier frequency are attenuated $1.5 \mathrm{~dB}$ within $0.2 \mathrm{~dB}$, using an $18 \mathrm{~dB}$ per octave design. Assuming a 1inear-phase design, a drift of jil the poles in frequency by $96 \mathrm{~Hz}$ will produce the $0.2 \mathrm{~dB}$ amplitude change and at the same time a shift of 5 degrees at the subcarrier frequency. This means a 0.1 percent frequency tolerance at $96 \mathrm{kHz}$. Although some samples have exhibited considerably more initial phase shift, this can be handled with adjustment, and the above variation with time and temperature will be assumed in calculations for the $96 \mathrm{kHz}$ airborne filter. Assuming the same percentage frequency change at the $64 \mathrm{kHz}$ pilot frequency, the same phase error (5 degrees) will appear on the regenerated $96 \mathrm{kHz}$ reference, and about 1 degree at the regenerated $16 \mathrm{kHz}$ reference input to the demodulator. The bandpass filters in the ground station will be assumed to contribute half as much uncompensated variation as those in the girborne unit.

It is assumed that the filters for the lower channels, including $16 \mathrm{kHz}$, will have looser percentage frequency drifts $(0.3$ percent airborne, 0.15 percent ground) than the $96 \mathrm{kHz}$ filter, as a matter of 
Table 3.5-1

Demodulator Steady Phase Errors-Harmonic Subcarriers

1. Phase error at $96 \mathrm{kHz}$ demodulator

Nonsystematic errors (Note A)

Airborne synthesizer at $64 \mathrm{kHz}$ (1)

Phase error

$1.0^{\circ}$

at $96 \mathrm{kHz}$ (2)

$1.0^{\circ}$

Airborne filter

at $64 \mathrm{kHz}$ (3)

$5.0^{\circ}$

at $96 \mathrm{kHz}$ (4)

$5.0^{\circ}$

Ground synthesizer

at $64 \mathrm{kHz}$ (1)

$1.0^{\circ}$

at $96 \mathrm{kHz}(2)$

$1.0^{\circ}$

Ground filter

at $64 \mathrm{kHz}$ (5)

$2.5^{\circ}$

at $96 \mathrm{kHz}$ (6)

$2.5^{\circ}$

Tape speed drift (0.1\%)

$0.7^{\circ}$

$7.9^{\circ}$

Root sum square

Systematic errors (Note B)

Radio link

$1.6^{\circ}$

Predetection tape link

Total for predetection tape mode

- Postdetection tape link (no data available)

Total for postdetection tape mode

\section{NOTES :}

A. Variations after initial adjustment to zero

B. With reference to $64 \mathrm{kHz}$ phase, without adjustment

1. $30 \mathrm{nsec}$ variation, referred to $96 \mathrm{kHz}$

2. $30 \mathrm{nsec}$ variation

3. Based on $0.1 \%$ drift, referred to $96 \mathrm{kHz}$

4. Based on $0.1 \% \mathrm{drift}$

5. Based on $0.05 \%$ drift, referred to $96 \mathrm{kHz}$

6. Based on $0.05 \%$ drift

7. Based on $20 \mu \mathrm{sec}$ filter delay difference

8. Based on Figure 2.5-1 curve 6

9. Based on Figure 2.5-1 curve 4 
Tab1e 3.5-1 (continued)

Demodulator Steady Phase Errors-Harmonic Subcarriers

2. Phase error at $16 \mathrm{kHz}$ demodulator

Nonsystematic errors (Note A)

Phase error

Airborne synthesizer at $64 \mathrm{kHz}$. (10)

$0.2^{\circ}$

at $16 \mathrm{kHz}$ (11)

$0.2^{\circ}$

Airborne filter

at $64 \mathrm{kHz}$ (12)

$1.0^{\circ}$

at $16 \mathrm{KH} \overline{\mathrm{s}}$ (13)

$3.0^{\circ}$

Ground synthesizer

at $64 \mathrm{kHz}(10)$

$0.2^{\circ}$

at $16 \mathrm{kHz}$ (11)

$0.2^{\circ}$

Ground filter

at $64 \mathrm{kHz}(14)$

$0.5^{\circ}$

at $16 \mathrm{kHz}$ (15)

$1.5^{\circ}$

Tape speed drift $(0.1 \%)$

$0.1^{\circ}$

Root sum square

$3.6^{\circ}$

Systematic errors (Note B)

Radio link

$0.0^{\circ}$

Predetection tape link

$0.0^{\circ}$

Total for predetection tape link

$3.6^{n}$

Postdetection tape link

$3.0^{\circ}$

Total for postdetection tape mode

$6.6^{\circ}$

NOTES :

(10) 30 nsec varlalluil, iefeired to $16 \mathrm{kll}$

(11) $30 \mathrm{nsec}$ variation

(12) Based on $0.1 \%$ drillt, referred to $16 \mathrm{kHz}$

(13) Based on $0.3 \%$ drift

(14) Based on $0.05 \%$ drift referred to $16 \mathrm{kHz}$

(15) Based on $0.15 \%$ drift

(16) Based on $20 \mu \mathrm{sec}$ filter delay difference

(17) Based on Figure 2.6-2 
economics. The total resulting error for $16 \mathrm{kHz}$ is estimated at 3.6 degrees for the predetection mode, with another 3 degrees from Figure 2.6-2 for the postdetection mode.

Another error will be caused by frequency drift in the airborne synthesizer oscillator, but this will likely be negligible. A similar effect takes place if there is a speed error (slowly varying or fixed) in the tape playback, so that all the frequencies are multiplied by the same constant. If the ground station is properly delay-compensated, no error results because all paths have the same phase vs frequency slope. For the sake of calculation, we will assume that there is an uncompensated delay variation between channels of 20 microseconds and that the tape speed is controlled to within 0.1 percent. The resulting error is 0.7 degrees at $96 \mathrm{kHz}$, and is not a matter of concern.

A11 of the above errors in the harmonic subcarrier system should not occur at their worst values simultaneously, and these estimates are combined in root-sum-square fashion in Table 3.5-1 to get an overall value; whose statistical qualities are left unspecified. This value is then added to systematic phase shifts expected from the radio and tape links.

The radio link phase shift is gotten from the model of curve 6 , Figure 2.5-1. The predetection tape link phase shift is assumed to be due mainly to a demodulator output lowpass filter as discussed in Section 2.6. Its value is taken from Figure 2.5-1, curve 4, assuming a $250 \mathrm{kHz}$ filter. The total value for the predetection mode is 12.1 degrees. This figure probably is optimistic in some components, pessemistic in others. The radio link and predetection link shifts can be reduced by adjustment, for instance, to pick up part of the 4.2 degrees listed for them in the table.

Postdetection recording for harmonic subcarriers gives an unknown phase shift at the high frequency end of the baseband, although the low end is covered in Section 2.6. A flyure of 7.2 degrees is assumed here at $96 \mathrm{kHz}$ for the sake of filling out the calculation table (it produces an overal1 figure of 0.3 for the tangent of the angle for Equation 3.1-11). This figure may seem a little large, but when one considers the severe amplitude variations over the baseband shown in Figure 2.6-1 it appears risky to assume a very small figure. At this time all we can 
say is that postdetection tape calculations for harmonic subcarriers are in the nature of an academic exercise, pending some measurement on combinations of machines comparable to the frequency response tests reported in Reference 10. 
4.0 Calculations.

Table 4.1-1

Baseband Noise Levels, $p_{2}$ and $p_{3}$ Values are Meansquare Spectra1 Density, in Hertz $2 / \mathrm{Hz}$ Equivalent

FM Carrier Modulation Density

\begin{tabular}{|c|c|c|c|c|c|}
\hline & $\begin{array}{l}\text { Pilot } \\
1.5 \mathrm{kHz} \\
\end{array}$ & $\begin{array}{l}\text { Pilot } \\
4 \mathrm{kHz} \\
\end{array}$ & $\begin{array}{l}\text { Channel } \\
16 \mathrm{kHz}\end{array}$ & $\begin{array}{l}\text { Pilot } \\
64 \mathrm{kHz}\end{array}$ & $\begin{array}{l}\text { Channel } \\
96 \mathrm{kHz}\end{array}$ \\
\hline Predet, record, $(\mathrm{S} / \mathrm{N})=12 \mathrm{~dB}$ & & & & & \\
\hline Radio link noise & 13 & 14 & 46 & 500 & 1090 \\
\hline Distortion & 20 & 20 & 23 & 63 & 117 \\
\hline Tape link noise & 3 & 1 & 1 & 1 & 3 \\
\hline Distortion & 6 & 6 & 6 & 6 & 6 \\
\hline Total & 42 & 41 & 76 & 570 & 1216 \\
\hline $\begin{array}{l}\text { Predet. record, }(\mathrm{S} / \mathrm{N})=39 \mathrm{~dB} \\
\quad \text { Radio link noise }\end{array}$ & 0 & 0 & 0 & 1 & 3 \\
\hline Distortion & 20 & 20 & 23 & 63 & 117 \\
\hline : Tape link noise & 3 & 1 & 1 & 1 & 3 \\
\hline Distortion & 6 & 6 & 6 & 6 & 6 \\
\hline Total & 29 & 27 & 30 & 71 & 129 \\
\hline $\begin{array}{l}\text { Postdet, record, }(\mathrm{S} / \mathrm{N})=12 \mathrm{~dB} \\
\text { Radio link noise }\end{array}$ & 13 & 14 & 46 & 500 & 1090 \\
\hline Distortion & 20 & 20 & 23 & 63 & 117 \\
\hline Tape link noise & 910 & 126 & 8 & 2 & 2 \\
\hline Distortion & 13 & 13 & 12 & 8 & 6 \\
\hline Total & 956 & 173 & 89 & 573 & 1215 \\
\hline $\begin{array}{l}\text { Postdet. record, }(\mathrm{S} / \mathrm{N})=39 \mathrm{~dB} \\
\text { Radio link noise }\end{array}$ & 0 & 0 & 0 & 1 & 3 \\
\hline Distortion & 20 & 20 & 23 & 63 & 117 \\
\hline Tape link noise & 910 & 126 & 8 & 2 & 2 \\
\hline Distortion & 13 & 13 & 12 & 8 & 6 \\
\hline Tota1 & 943 & 159 & 43 & 74 & 128 \\
\hline
\end{tabular}

Densities are taken from Figure 1.4-1, based on:

Fig. 2.2-1 For radio link noise

Fig. 2.2-5 For radio link distortion

Fig. 2.3-2 For predet. tape link noise

Fig. 2.3-3 For predet. tape link distortion

Fig. 2.3-1 For postdet. tape link noise

Fig. 2.3-1 For postdet. tape link distortion 
Table 4.1-2

Calculations

Additive Noise

$\mathrm{R}^{2}=\mathrm{P}_{2} \mathrm{~B}_{6} / 4 \mathrm{P}_{2}$

From Equation 3.1-7

$B_{6}=1000 \mathrm{~Hz}$

$\mathrm{P}_{2}$ from Table 4.1-1

$\mathrm{P}_{2}$ from Table 1.3-1

\begin{tabular}{|c|c|c|c|c|c|c|c|}
\hline $\begin{array}{l}\text { Mux } \\
\text { eype }\end{array}$ & $\begin{array}{l}\text { Record } \\
\text { type }\end{array}$ & $\begin{array}{c}(\mathrm{S} / \mathrm{N})_{1} \\
\mathrm{~dB}\end{array}$ & $\begin{array}{c}\text { Channel } \\
\mathrm{kHz}\end{array}$ & $\mathrm{Hz}^{2} / \mathrm{Hz}$ & $\begin{array}{l}\mathrm{P}_{2} 2 \\
\mathrm{~Hz}\end{array}$ & $\mathrm{R}^{2}$ & $\begin{array}{r}100 \mathrm{R} \\
\%\end{array}$ \\
\hline \multirow{8}{*}{ Indep. } & Fredet. & 12 & \multirow[t]{2}{*}{16} & 76 & \multirow[t]{2}{*}{$655 \times 10^{6}$} & $29 \times 10^{-6}$ & 0.54 \\
\hline & & 39 & & 30 & & 11.5 & 0.34 \\
\hline & & 12 & \multirow[t]{2}{*}{96} & 1215 & & 464 & 2.16 \\
\hline & $\downarrow$ & 39 & & 129 & & 48.2 & 0.70 \\
\hline & Postdet. & 12 & \multirow[t]{2}{*}{16} & 89 & & 34.0 & 0.58 \\
\hline & & 39 & & 43 & & 16.4 & 0.41 \\
\hline & & 12 & \multirow[t]{2}{*}{96} & 1215 & & 465 & 2.16 \\
\hline & V & 39 & & 128 & & 48.7 & 0.70 \\
\hline \multirow[t]{8}{*}{ Harm. } & Predet. & 12 & \multirow[t]{2}{*}{16} & 76 & $697 \times 10^{6}$ & 27.3 & 0.52 \\
\hline & & 39 & & 30 & & 10.8 & 0.33 \\
\hline & & 12 & \multirow[t]{2}{*}{96} & 1216 & & 436.5 & 2.09 \\
\hline & $\downarrow$ & 39 & & 129 & & 46.3 & 0.68 \\
\hline & Pustdet. & 12 & \multirow[t]{2}{*}{16} & 89 & & 32.0 & 0.57 \\
\hline & & 39 & & 43 & & 15.4 & 0.39 \\
\hline & \multirow{2}{*}{$\downarrow$} & 12 & \multirow[t]{2}{*}{96} & 1215 & & 436.2 & 2.09 \\
\hline & & 39 & & 128 & 1 & 46.0 & 0.68 \\
\hline
\end{tabular}




\section{Table 4.1-3 \\ Calculations}

Baseband Level Control Noise

$$
\begin{aligned}
& \mathrm{R}^{2}=\frac{\mathrm{e}_{6} \mathrm{p}_{3} \mathrm{~B}_{\mathrm{b}}}{4 \mathrm{E}_{6} \mathrm{P}_{3}} \\
& \mathrm{~B}_{\mathrm{b}}=126 \mathrm{~Hz}
\end{aligned}
$$

From Equation 3.3-7

\begin{tabular}{|c|c|c|c|c|c|c|}
\hline $\begin{array}{l}\text { Mux. } \\
\text { type }\end{array}$ & $\begin{array}{c}\text { Record } \\
\text { type }\end{array}$ & $\begin{array}{c}\text { Carrier } \\
\text { noise } \\
\mathrm{dB}\end{array}$ & $\begin{array}{l}\mathrm{B}_{\mathrm{b}} \\
\mathrm{Hz}\end{array}$ & $\begin{array}{c}\mathrm{P}_{3} \\
\mathrm{~Hz}^{2} / \mathrm{Hz} \\
\end{array}$ & $\begin{array}{r}\mathrm{P}_{3} \\
\mathrm{~Hz} \\
\end{array}$ & $\mathrm{R}^{2}$ \\
\hline Indep. & Predet. & 12 & 126 & 42 & $328 \times 10^{6}$ & $4.0 \times 10^{-6}$ \\
\hline & & 39 & & 29 & & 2.8 \\
\hline & Postdet. & 12 & & 956 & & 91.8 \\
\hline & & 39 & & 943 & & 90.5 \\
\hline Harm. & Predet. & 12 & & 41 & $174 \times 10^{6}$ & 7.4 \\
\hline & & 39 & & 27 & & $4.9 \times 10^{-6}$ \\
\hline & Postdet. & 12 & & 173 & & 3.13 \\
\hline & 1 & 39 & & 159 & $\nabla$ & 28.8 \\
\hline
\end{tabular}

Test channel output $e_{6}=E_{6}$

P3 from Table 4.1-1, at $1.5 \mathrm{kHz}$ for independent subcarriers, $4.0 \mathrm{kHz}$ for harmonic subcarriers

$\mathrm{P}_{3}$ from Table 1.3-1 


\section{Table 4.1-4}

Calculations

Phase Lock Loop Noise

$\mathrm{R}^{2}=\frac{1}{4}\left[\frac{\phi_{\mathrm{b}}{ }^{4}}{2}+\phi_{\mathrm{b}}{ }^{2} \tan ^{2} \theta_{\mathrm{a}}\right]$ from Equation $3.1-11$, Figure 3.1-1

$\phi_{\mathrm{b}}^{2}=\phi_{5}^{2}=\left(\mathrm{f}_{2} / \mathrm{f}_{3}\right)^{2} \mathrm{p}_{3} \mathrm{~B}_{\mathrm{L}} / \mathrm{P}_{3}$ from Equation $3.2-13$

For independent subcarriers:

Test channel ourpur $\mathrm{e}_{6}=\mathrm{E}_{6}$

For harmonic subcarriers:

Test channel output $\mathrm{e}_{6}=\mathrm{E}_{6}$ $f_{3}=f_{2}, p_{3}=p_{2}, P_{3}=P_{2}$

$\mathrm{B}_{\mathrm{L}}=4 / 1 \mathrm{~Hz} \quad \mathrm{P}_{2}=655 \times 10^{6} \mathrm{~Hz}^{2}$

$\mathrm{f}_{3}=64 \mathrm{kHz} \quad \mathrm{B}_{\mathrm{L}}=471 \mathrm{~Hz}$

$\mathrm{P}_{3}=174 \times 10^{6} \mathrm{~Hz}^{2}$

$\mathrm{P}_{3}$ from Table 4.1-1, $\mathrm{P}_{2}$ from Table $1.3-1, \theta_{\text {a }}$ from Table 3.5:1

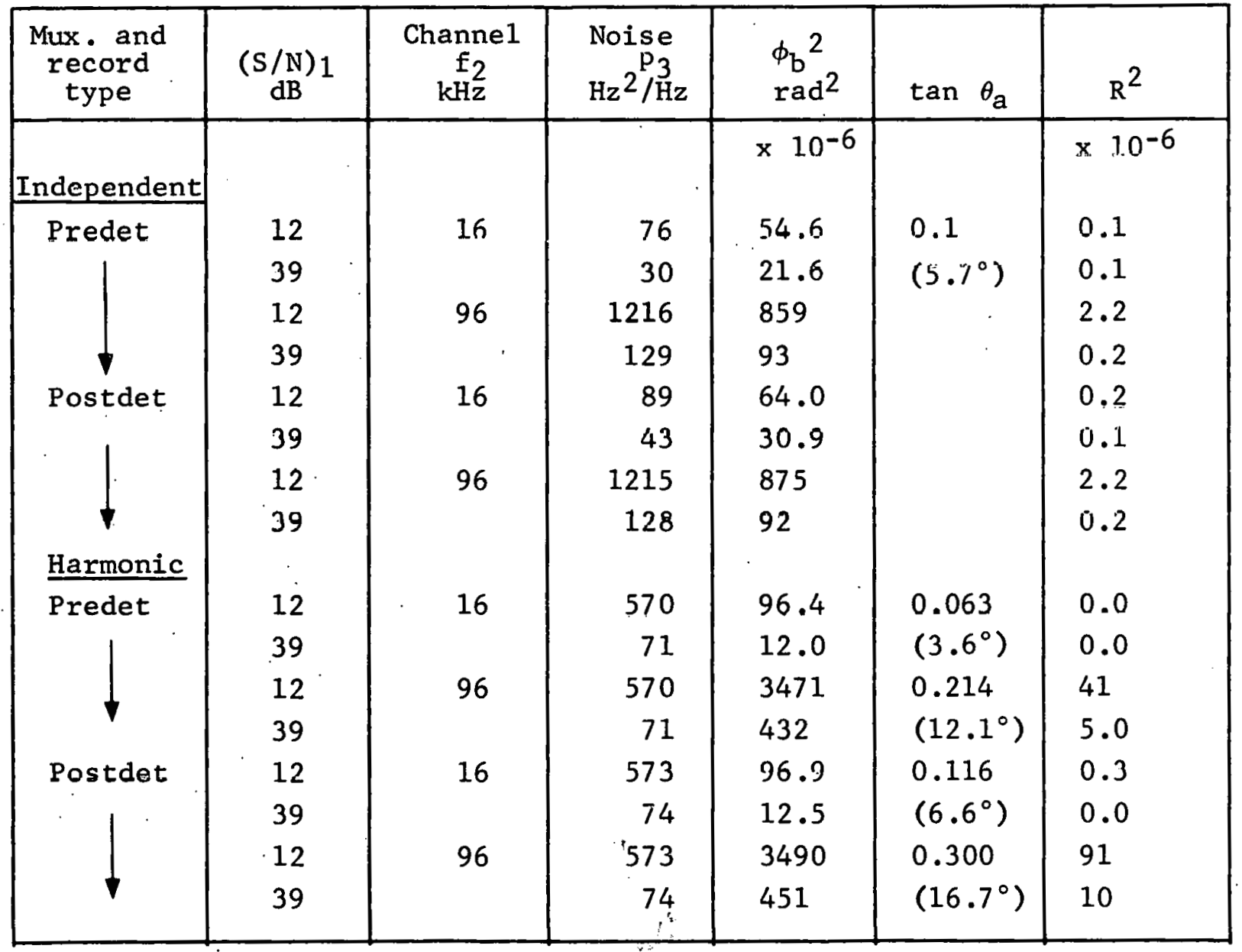


Table 4.1-5

Calculation

Magnetic Tape Link Time Base Error

Parameters from Table 1.7-1

PLL $\mathrm{f}_{\mathrm{n}}=150 \mathrm{~Hz}$

$$
\begin{array}{ll}
\mathrm{R}^{2}=\frac{1}{4}\left[\frac{\phi_{\mathrm{b}}{ }^{4}}{2}+\phi_{\mathrm{b}}{ }^{2} \tan ^{2} \theta_{\mathrm{a}}\right] & \text { From Equation } 3.1-11, \mathrm{Fig} \cdot 3.1-1 \\
\phi_{\mathrm{b}}{ }^{2}=\phi_{5}{ }^{2}=\left(2 \pi \mathrm{f}_{2} \mathrm{~T}\right)^{2} & \text { From Equation } 3.2-20 \\
\mathrm{~T}^{2}=\mathrm{K}_{\mathrm{z}}{ }^{2} \frac{-4 \mathrm{f}_{\mathrm{n}}^{\mathrm{n}+1}}{(\mathrm{n}+5)(\mathrm{n}+1)} & \text { From Equation } 3.2-18, \mathrm{Fig} \cdot 3.2-3
\end{array}
$$

\begin{tabular}{|c|c|c|c|c|c|c|c|c|}
\hline $\begin{array}{c}\text { Chan. } \\
\mathrm{f}_{2} \\
\mathrm{kH} z\end{array}$ & $\begin{array}{l}\text { Record } \\
\text { mode }\end{array}$ & $\begin{array}{c}\text { TBE } \\
\text { mode1 }\end{array}$ & $\begin{array}{c}\theta_{a} \\
\text { Degrees }\end{array}$ & $\tan \theta_{a}$ & $\underset{\text { Radian }}{\phi_{b}}$ & $\begin{array}{c}\boldsymbol{\Phi}_{\mathrm{b}}^{2} \\
\operatorname{Radian}^{2}\end{array}$ & $\mathrm{R}^{2}$ & $100 \mathrm{R}$ \\
\hline \multicolumn{3}{|c|}{ For Harmonic Subcarriers: } & & & $\times 10^{-3}$ & $\times 10^{-6}$ & $\times 10^{-6}$ & \\
\hline 96 & $\begin{array}{l}\text { Predet } \\
\text { Postdet } \\
\text { Predet } \\
\text { Postdet }\end{array}$ & $\begin{array}{l}\text { A1 } \\
\text { A2 } \\
\text { B } \\
\text { C } \\
\text { A1 } \\
\text { A2 } \\
\text { B } \\
\text { C } \\
\text { A1 } \\
\text { A2 } \\
\text { B } \\
\text { C } \\
\text { A1 } \\
\text { A2 } \\
\text { B } \\
\text { C }\end{array}$ & $\begin{array}{l}12.1 \\
16.7 \\
3.6\end{array}$ & $\begin{array}{l}0.300 \\
0.063\end{array}$ & $\begin{array}{l}133 \\
66.4 \\
90.5 \\
187 \\
133 \\
66.4 \\
90.5 \\
187 \\
22 \\
11.1 \\
15.1 \\
31.2 \\
22 \\
11.1 \\
15.1 \\
31.2\end{array}$ & $\begin{array}{l}17600 \\
4400 \\
8190 \\
35000 \\
17600 \\
4400 \\
8190 \\
35000 \\
483 \\
123 \\
228 \\
973 \\
483 \\
123 \\
228 \\
973\end{array}$ & $\begin{array}{l}225 \\
54 \\
105 \\
560 \\
435 \\
102 \\
200 \\
940 \\
0.5 \\
0.1 \\
0.2 \\
1.1 \\
1.7 \\
0.4 \\
0.8 \\
3.4\end{array}$ & $\begin{array}{l}1.50 \\
0.74 \\
1.03 \\
2.37 \\
2.09 \\
1.01 \\
1.41 \\
3.07 \\
0.07 \\
0.03 \\
0.04 \\
0.10 \\
0.13 \\
0.06 \\
0.09 \\
0.18\end{array}$ \\
\hline \multicolumn{9}{|c|}{ For Independent Subcarriers: } \\
\hline 96 & - & $\begin{array}{l}\text { A1 } \\
\text { A2 } \\
\text { B } \\
\text { C } \\
\text { A1 } \\
\text { A2 } \\
\text { B } \\
\text { C }\end{array}$ & 5.7 & 0.1 & $\begin{array}{l}133 \\
66.4 \\
90.5 \\
187 \\
22 \\
11.1 \\
15.1 \\
31.2\end{array}$ & $\begin{array}{l}17600 \\
4400 \\
8190 \\
35000 \\
483 \\
123 \\
228 \\
973\end{array}$ & $\begin{array}{l}81 \\
13.7 \\
29.0 \\
234 \\
1.2 \\
0.3 \\
0.6 \\
2,6\end{array}$ & $\begin{array}{l}0.9 \\
0.37 \\
0.54 \\
1.53 \\
0.11 \\
0.05 \\
0.08 \\
0.16\end{array}$ \\
\hline
\end{tabular}

$\theta_{\text {a }}$ from Table 3.5-1, $\mathrm{K}_{z}$ and $\mathrm{n}$ from Figure 2.4-5 


\section{REFERENCES}

1. F. Schmitt, "Double Sideband (DSB) Telemetry Study," Lockheed Electronics Technical Report 68-1, January 1968. Prepared by Lockheed for Data Systems Division, Instrumentation Development Directorate, National Range Engineering, White Sands Missile Range, New Mexico 88002

2. M. H. Nichols and F. J. Schmitt, "Cause and Effect of Time Base Errors in Coherent Demodulation of a Suppressed Carrier AM Multiplex," Proceedings of the 1968 International Telemetering Conference, pp. 399-406

3. W. E. Thompson, "The Response of a Nonlinear System to Random Noise," IEE Monograph No. 106R, September 1954

4. A. F. Ghais, E. J. Ferrari, and C. J. Boardman, "Study of Telemetry Receiver and Recorder Phase Linearity Problems," prepared for Air Force Systems Command by ADCOM, Inc., August $1966^{\circ}$

5. K. M. Uglow, "The Effect of Receiver Bandwidth on Intermodulation in FDM/FM Systems," Technical Note 6803, March 25, 1968

6. C. H. Chen, R. J. D'Auteuil, and R. O'Keefe, "Computer Optimization of FM/FM Telemetry Systems," Proceedings of the 1969 National Telemetering Conference, pp. 234-240

7. C. S. Johnson and R. M. Caster, "Nonlinear Effects in Telemetry Transmitter-Receiver RF Links," Proceedings of the 1969 Nationa1 Telemetering Conference, pp. 15-21

8. C. S. Johnson and R. M. Caster, "Nonlinear Effects in Telemetry Transmitter-Receiver RF Links," Sandia Report SC-RR-69-138, May 1969, Sandia Laboratories, Albuquerque; New Mexico

9. W. H. Tranter and G. D. Weathers, "An Investigation of Baseband Phase Characteristics of an FM Transmission Link," Sperry Rand Report, September 20, 1968

10. G. H. Schulze, "Compatibility Requirements and Considerations of Range Telemetry Tape," Proceedings of 1968 International Telemetering Conference, pp. 378-391

11. E. B. Campbe11 and W. R. Herbert, "Telemerty FM/FM Baseband Structure Study," Vo1. I and II, EMR Report prepared for. White Sands Missile Range, June 14, 1965

12. E. B. Richardson and P.F. Harney, "F1ight and Laboratory Testing of a Double Sideband FM Telemetry System," Proceedings of the 1968 Internati ona1 Telemetering Conference, pp. 581-596

13. F. M. Gardner, Phaselock Techniques, John Wiley and Sons, New York, 1966

14. R. W. Sanneman and J. R. Rowbotham, "Unlock Characteristics of the Optimum Type II Phase-Locked Loop," IEEE Transactions on Aerospace and Navigational Electronics, March 1964, pp. 15-24

15. W. H. Tranter, R. S. Simpson, and W. O. Frost, "Considerations in the Use of AGC in AM/FM Telemetry Systems," Telemetry Journal, February/March 1969, pp. 19-23 
DISTRIBUTION:

Clearinghouse for

Federal Scientific and Tech Info

Springfield, Virginia 22151 (15)

Dr. Walter Hedeman

Aerospace Corporation

P.0. Box 1308

San Bernardino, California 92402

Mr. K. L. Berns

Code 42302

Naval Weapons Corona Laboratories

Corona, California 91720

$\mathrm{Mr}$. John B. Venters

Instrumentation Branch

Army Inertial G\&C Lab., MICOM

U. S. Army Missile Command

Redstone Arsenal, Alabama 35809

Mr. George C. Thomas

MU 707, Building 989, Box 4187

Pan American World Airways

Patrick AFB, Florida 32925

Mr. Raymond A. Runyan

Data-Control Systems, Inc.

E. Liberty Street

Danbury, Connecticut

Mr. Martin Belkin

EMR-Telemetry

P.0. Box 3041

Sarasota, Florida 33578

Mr. Walter 0. Frost

Chief, Telemetry and Data Technology Branch NASA/Marshall Space Plight Center

Huntsvilie, Alabama 35812

Mr. Lawrence W. Gardenhire

Radiation, Inc.

P.0. Box 37

Melbourpe, Florida

Mr. Robert C. Barto

Chief, Data Collection Systems Division

Range Instrumentation Systems Office

White Sands Missile Range

New Mexico 88002
Mr. B. E. Norman

STEWS-RE-SE

White Sands Missile Range

New Mexico 88002

Mr. J. R. Devilbiss

STEWTS-NR-DE-T

White Sands Missile Range

New Mexico 88002

Mr. F. T. Sinnott

Aerospace Corporation

P.0. Box 95085

Ios Angeles; Callfornia 90045

Mr. J. C. Greenfield

TAWL, Bldg. 65

Picatinny Arsenal

Dover, New Jersey 07801

Mr. D. Ray Andelin

McDonnell-Douglas Astronautics

Western Division

5301 Bolse

Huntington Beach, California 92646

Mr. Jacky Cates

White Sands Missile Range

New Mexico 88002

Mr. Hugh Pruss

Teledyne Telemetry

9320 Iincoln Boulevard

Ios Angeles, Callfornia

Mr. J. H. Billups

Code 400533

Naval Weapons Center

China Lake, California 93555

Mr. Victor W. Hammond

Office, Secretary of Defense

Washington, D. C .

Dr. Myron H. Nichols

2682 Idle Hour Lane

LoJolla, California 92037

Mr. Kenneth M. Uglow

P. O. Box 2260

Sarasota, Florida 33578
(10 copies) 
DISTRIBUTION: (cont)

Mr. George Gregory

MDTI-2

Air Force Missile Development Center

Holloman AFB, New Mexico 88330

Mr. W. B. Poland, Jr.

Code 563

INASA, Goddard Space Flight Center

Greenbelt, Maryland 20771

Mx. I. Arsement

R-ASTR-ITE

NASA, Marshall Space Flight Center

Huntsville, Alabama 35808

Mr. Edward Campbell

Sonex, Inc.

20 E. Herman Street

Philadelphia, Penn. 19144

Dr. Jack H. Crow

Vice President of Engineering

Defense Electronics, Inc.

Rockville, Maryland 20854

$M r$. R. B. Richardson

MASA, Flight Research Center

P.0. Box 273

Edwarda, California 93523

Mr. Ronald M. Muller

NASA, Goddard Space Flight Center

Greenbelt, Maryland 20772

Mr. Joseph K, Koukol

Jet Propulsion Laboratory

Pasadena, California

Mr. T. W. Alexander

STEWS-RE-TD-C

White Bauds Missile Range

New Mexico 88002
L. E. Hollingsworth, 7200

T. L. Pace, 9220

0. M. Steutzer, 7210

G. R. Bachand, 7211

R. S. Millican, 7221

C. S. Johnson, 7221

H. 0. Jeske, 7221

J. G. Hawley, 7222

W. K. Paulus, 7293

J. E. Stiegler, 7280

S. A. Ingham, 9125

A. E. Bentz, 7282

T. J. Hoban, 7283

M. L. Kramm, 9210

R. S. Reynolds, 9213

J. C. Eckhart, 9220

H. H. Patterson, 9230

Central Files, 3422 (25)

G. C. McDonald, 3416 (4)

Attn: P. Swartz 